TRANSACTIONS OF THE

AMERICAN MATHEMATICAL SOCIETY

Volume 353, Number 8, Pages 2983-3043

S 0002-9947(01)02769-6

Article electronically published on March 22, 2001

\title{
CONSTRUCTION AND ASYMPTOTIC STABILITY OF STRUCTURALLY STABLE INTERNAL LAYER SOLUTIONS
}

\author{
XIAO-BIAO LIN
}

\begin{abstract}
We introduce a geometric/asymptotic method to treat structurally stable internal layer solutions. We consider asymptotic expansions of the internal layer solutions and the critical eigenvalues that determine their stability. Proofs of the existence of exact solutions and eigenvalue-eigenfunctions are outlined.

Multi-layered solutions are constructed by a new shooting method through a sequence of pseudo Poincaré mappings that do not require the transversality of the flow to cross sections. The critical eigenvalues are determined by a coupling matrix that generates the SLEP matrix. The transversality of the shooting method is related to the nonzeroness of the critical eigenvalues.

An equivalent approach is given to mono-layer solutions. They can be determined by the intersection of a fast jump surface and a slow switching curve, which reduces Fenichel's transversality condition to the slow manifold. The critical eigenvalue is determined by the angle of the intersection.

We present three examples. The first treats the critical eigenvalues of the system studied by Angenent, Mallet-Paret \& Peletier. The second shows that a key lemma in the SLEP method may not hold. The third is a perturbed activator-inhibitor system that can have any number of mono-layer solutions. Some of the solutions can only be found with the new shooting method.
\end{abstract}

\section{INTRODUCTION}

The existence of sharp internal layer solutions is an important feature in singularly perturbed parabolic systems. These systems are characterized by the drastic difference in diffusion rates of the two equations and have found applications in the fields of chemical reactions, morphogenesis, solidification, etc. We consider systems that can be written in a fast-slow form with appropriate boundary conditions at $x=0,1$,

$$
\begin{aligned}
& u_{t}=\epsilon^{2} u_{x x}+f(u, v), \quad u \in \mathbb{R}^{m}, v \in \mathbb{R}^{n}, \\
& v_{t}=v_{x x}+g(u, v), \quad 0<x<1 .
\end{aligned}
$$

Received by the editors March 18, 1998 and, in revised form, May 5, 2000.

2000 Mathematics Subject Classification. Primary 34D15, 34E05, 35B25; Secondary 35C20, 37C29, 34C37.

Key words and phrases. Singular perturbation, matched asymptotic expansion, internal layers, critical eigenvalues, stability, structural stability.

Research partially supported by NSF grant 9501255 . 
A well-known example of the above is the activator-inhibitor model [35, 37] where $f$ and $g$ are scalar functions.

$$
\begin{aligned}
& f(u, v)=u-u^{3}-v, \\
& g(u, v)=a_{0} u-a_{1} v, \\
& u_{x}=v_{x}=0, \quad x=0,1 .
\end{aligned}
$$

The existence of mono-internal layer solutions for such models were studied by many authors [12, 14, 23, 36]. Multi-layered solutions can be constructed by a folding up principle [40, 38, 46]. The stability of both mono and multi-layered solutions was first proved by Nishiura and Fujii [39, 40. For brevity, (1.2) will be called the NF model.

Another well studied model is the $x$-dependent scalar equation

$$
\begin{aligned}
u_{t} & =\epsilon^{2} u_{x x}+\left(1-u^{2}\right)(u-a(x)), \quad 0<x<1, \\
u_{x} & =0, \quad x=0,1,
\end{aligned}
$$

where $a(x)$ is a $C^{\infty}$ function with $a\left(x^{i}\right)=0, a^{\prime}\left(x^{i}\right) \neq 0$ at points $0<x^{1}<x^{2}<$ $\cdots<x^{r}<1$. By letting $v=x$ which satisfies $v_{t}=v_{x x}, v(0)=0, v(1)=1$, (1.3) can be converted into (1.1) with $g(u, v)=0$. Equation (1.3) also has a long history [11, 19]. Very general results of (1.3) were obtained by Angenent, Mallet-Paret \& Peletier [1]. (1.3) will be called the AMP model.

We will only consider stationary internal layer solutions to (1.1). They satisfy the system

$$
\begin{aligned}
& 0=\epsilon^{2} u_{x x}+f(u, v), \\
& 0=v_{x x}+g(u, v), \quad 0<x<1 .
\end{aligned}
$$

In both the NF and AMP models, the singular limit of the internal layer solutions can be described as follows. $f(u, v)=0$ has three branches of solutions $u=$ $h_{ \pm}(v)$ and $u=h_{0}(v)$. The branches $u=h_{ \pm}(v)$ consist of stable equilibria of the reaction equation, $u_{t}=f(u, v)$, where $v$ is a parameter. The branch $u=h_{0}(v)$ is unstable for the same ODE. An internal layer solution $(u(x), v(x))$ stays near the two slow manifolds $u=h_{ \pm}(v)$ for most of the points $x$. These $x$ form the regular layers. Exceptional points are near a finite sequence $\left\{x^{i}\right\}_{1}^{r}$, where the solution jumps between the two slow manifolds. These points form the internal layers. They are also the places where $\epsilon^{2} u_{x x}$ is no longer negligible.

In the singular layer near $x^{i}$, the stretched variable $\xi=\left(x-x^{i}\right) / \epsilon$ is introduced. If $\epsilon=0$, the $u$-equation becomes

$$
u_{\xi \xi}+f(u, \bar{v})=0 .
$$

The condition on the parameter $\bar{v}=v\left(x^{i}\right)$ is that (1.5) must have a heteroclinic solution connecting the two slow manifolds $u=h_{ \pm}(\bar{v})$.

In the regular layers, when $\epsilon=0, v$ satisfies

$$
v_{x x}+g(h(v), v)=0, \quad h(v)=h_{ \pm}(v),
$$

with boundary conditions at $x=0,1$. Let $x^{0}=0$ and $x^{r+1}=1$. Then $v$ is $C^{1}$ in $[0,1]$ and is $C^{2}$ in each $\left(x^{i}, x^{i+1}\right)$. When $x$ crosses $x^{i}, 1 \leq i \leq r, h(v)$ switches between $h_{-}(v)$ and $h_{+}(v)$.

It is known that in the NF model, there is only one mono-layer solution which jumps from near $u=h_{-}(v)$ to near $u=h_{+}(v)$. This solution is stable as a 
stationary solution to (1.1). In the AMP model, there can be many such monolayer solutions and their stability is determined by the signs of $a^{\prime}\left(x^{i}\right)$ where $x^{i}$ 's are the layer positions. Comparing the multi-layered solutions, we find that in the AMP model, the layer positions are determined by the zeros of $a(x)$, while in the NF model, multiple layers and the layer positions are constructed by a folding up method [40, 38, 46].

Although internal layer solutions in the NF and AMP model differ in many ways, they are structurally stable solutions that persist under small perturbations of $f$ and $g$. To see structurally unstable internal layer solutions, let us consider the following example,

$$
\begin{aligned}
& 0=\epsilon^{2} u_{x x}+f(u, \alpha y+\beta a(x)), \\
& 0=y_{x x}+g(u, y) .
\end{aligned}
$$

$a(x)$ is as in (1.3) and $g(u, y)=a_{0} u-a_{1} y$. The function $f(u, y)$ can be $\left(1-u^{2}\right)(u-y)$ or $u-u^{3}-y$. By setting $v=(y, x)$, (1.7) becomes (1.4) with two slow variables. The constants $\alpha$ and $\beta$ are parameters. If $0 \leq \alpha \leq 1, \beta=1-\alpha$, (1.7) provides a homotopy between the two types of systems. When moving $\alpha$ from 1 to 0 , the system moves from the NF type to the AMP type, and mono-layered solutions will be created through some structurally unstable solutions.

We consider structurally stable internal layer solutions and their stability under the assumption that the slow manifolds are normally hyperbolic. Thus, internal layer solutions in Van der Pol's equation and some other equations with turning points will not be considered although they are structurally stable. The structural stability in this paper relies on some transversality conditions which we believe to be optimal in the sense that further weakening of these conditions would cause the solutions to be structurally unstable. The understanding of the bifurcation of internal layer solutions is far from complete and will not be touched in this paper. A special example of bifurcation of internal layer solutions, caused by violation of the conditions of this paper has been studied by Hale and Lin [18].

We now preview some results in this paper.

In $\S 2$, we state some basic notations, definitions and lemmas.

In the first part of $\S 3$, we present a shooting method that determines singular internal layer solutions. Since (1.4) can be converted into a singularly perturbed first order system which has been treated before [30, 48], to compare our method with the one used in [30], we first describe a geometric method from [30].

Let us consider the perturbed NF model (1.7) with the Neumann boundary conditions at $x=0,1$. Suppose $(u(x, \epsilon), y(x, \epsilon))$ is an internal layer solution with layers near $\left\{x^{i}\right\}_{1}^{r}$. Let $x^{0}=0, x^{r+1}=1$. On the regular layers, the solution is near the slow manifolds $u=h_{ \pm}(\alpha y+\beta a(x))$. At the singular limit $\epsilon=0, y$ should satisfy

$$
y_{x x}+g\left(h_{ \pm}(\alpha y+\beta a(x)), y\right), \quad 0<x<1,
$$

with $y_{x}(0)=y_{x}(1)=0$. Alternating signs of \pm must be used on successive intervals $\left(x^{i}, x^{i+1}\right)$. The solution is $C^{1}$ throughout $[0,1]$ and is $C^{2}$ on each $\left(x^{i}, x^{i+1}\right)$. At $x^{i}, 1 \leq i \leq r, y_{x x}$ has a first kind of jump.

When $f(u, v)=u-u^{3}-v$, (1.5) has heteroclinic solutions between the two equilibria $h_{ \pm}(\bar{v})$ if and only if $\bar{v}=0$. Therefore, one of the conditions on $x^{i}, 1 \leq$ $i \leq r$, is that $\alpha y\left(x^{i}\right)+\beta a\left(x^{i}\right)=0$. 
The other condition on $x^{i}$ comes from the boundary conditions that $y(x, \epsilon)$ must satisfy. Let us rewrite (1.8) as a first order system

$$
\begin{aligned}
& \frac{d y}{d t}=z, \\
& \frac{d z}{d t}=g(h(\alpha y+\beta a(x)), y), \quad h=h_{ \pm}, \\
& \frac{d x}{d t}=1 .
\end{aligned}
$$

Define

$$
\begin{aligned}
\Gamma^{i} & =\{(y, z, x): \alpha y+\beta a(x)=0\}, \quad i=1, \ldots, r, \\
\Gamma^{0} & =\{(y, z, x): x=0\}, \quad \Gamma^{r+1}=\{(y, z, x): x=1\}, \\
\mathcal{S}_{0} & =\{(y, z, x): z=0, x=0\}, \\
\mathcal{S}_{1} & =\{(y, z, x): z=0, x=1\} .
\end{aligned}
$$

The surface $\Gamma^{i}, i=1, \ldots, r$, where a fast jump of $u$ can occur, is called the fast jump surface. A solution of the boundary problem (1.8) must start at $\mathcal{S}_{0}$ and end at $\mathcal{S}_{1}$. At first, $h$ can either be $h_{-}$or $h_{+}$; then each time $(y, z, x)$ hits $\Gamma^{i}, i=1, \ldots, r$, $h$ switches between $h_{-}$and $h_{+}$.

The following hypothesis was imposed in [30].

Transversality Hypothesis. In the regions near a specific orbit $(y(t), z(t), x(t))$ of (1.9), the flow of (1.9) intersects transversely with each $\Gamma^{i}$.

By the Transversality Hypothesis, the Poincaré mapping $\mathcal{P}^{i}: \Gamma^{i} \rightarrow \Gamma^{i+1}$ can be defined for each $0 \leq i \leq r$. Assume that the image of $\mathcal{S}_{0}$ under the composite mapping $\mathcal{P}^{r} \ldots \mathcal{P}^{1} \cdot \mathcal{P}^{0}$ intersects transversely with $\mathcal{S}_{1}$ in $\Gamma^{r+1}$. This intersection determines $(y(1), z(1))$. By applying the inverse mappings of $\mathcal{P}^{r}, \cdots, \mathcal{P}^{0}$ to $(y(1), z(1), 1)$, we find all the switching points $\left(y\left(x^{i}\right), z\left(x^{i}\right), x^{i}\right)$ and hence the solution $(y, z, x)$.

The necessity of the Transversality Hypothesis was questioned by K. Palmer in a personal conversation. By studying the perturbed NF model in $\S 6$, I have confirmed that the hypothesis is too strong. There exist structurally stable monolayer solutions where the reduced flow in the slow variables is not transverse to the fast jump surface at the intersection. See Figure 6.5 and Remark (iv) at the end of $\S 6$.

The new shooting method presented in $\S 3$ does not need the Transversality Hypothesis. Under some mild conditions, (H4)-(H6), a sequence of pseudo Poincaré mappings $\left\{\mathcal{P}^{i}\right\}_{0}^{r}$ can be defined. The mapping $\mathcal{P}^{i}$ is not a diffeomorphism from $\Gamma^{i}$ to $\Gamma^{i+1}$, however, the image of $\mathcal{S}_{0}$ under the composite mapping $\mathcal{P}^{r} \ldots \mathcal{P}^{1} \cdot \mathcal{P}^{0}$ is well defined. The transverse intersection of $\left(\mathcal{P}^{r} \ldots \mathcal{P}^{1} \mathcal{P}^{0}\right) \mathcal{S}_{0}$ with $\mathcal{S}_{1}$ uniquely determined the solution $(y, z, x)$.

In the second part of $\S 3$, we give a procedure to compute matched asymptotic expansions of the internal layer solutions to any order of $\epsilon$. Our calculation relies on solving a reduced system of boundary value problems with interface conditions (BVPIC), which has a unique solution under conditions (H1)-(H6). In $\S 7$, we show, that under an additional condition (H7), the (BVPIC) can be solved if the SLEP matrix, due to Nishiura and Fujii [40, 38, 46], is nonsingular.

Higher order expansions provide more precise approximations to the internal layer solutions and are easier to obtain than exact solutions. For this reason, the 
asymptotic method has always been an important way to solve singular perturbation problems. The expansion of internal layer solutions will also be used to obtain the expansion of critical eigenvalues.

There is a close relation between computing the formal and the exact solutions. Let $(U, V)=\left(\sum_{0}^{m} \epsilon^{j} U_{j}, \sum_{0}^{m} \epsilon^{j} V_{j}\right)$ be a formal internal layer solution, and let $(U+$ $\Delta U, V+\Delta V)$ be an exact solution. Then $(\Delta U, \Delta V)$ satisfies a system of equations similar to that satisfied by $\left(U_{j}, V_{j}\right), j \geq 1$. If we know how to calculate higher order terms $\left(U_{j}, V_{j}\right)$, under the same conditions, we should be able to calculate the correction $(\Delta U, \Delta V)$. The idea will be outlined in the Appendix. See also [18].

In $\S 4$ of this paper, we introduce a method to compute asymptotic series of critical eigenvalues and corresponding eigenfunctions of the internal layer solutions. Our approach can be used on some systems not covered by the SLEP method due to Nishiura and Fujii [40, 38, 46].

Nishiura and Fujii have shown that the stability of internal layer solutions of the $\mathrm{NF}$ model is determined by $r$ critical eigenvalues $\lambda(\epsilon)=\epsilon \lambda_{1}+O\left(\epsilon^{2}\right)$, where $r$ is the number of internal layers and the coefficient $\lambda_{1}$ is an eigenvalue of the so-called SLEP matrix.

Consider the eigenvalue problem

$$
\begin{aligned}
& \lambda U=\epsilon^{2} U_{x x}+f_{u} U+f_{v} V, \\
& \lambda V=V_{x x}+g_{u} U+g_{v} V,
\end{aligned}
$$

where $(U, V)$ is the eigenfunction corresponding to the eigenvalue $\lambda$, and the coefficients of $U$ and $V$ are evaluated on the internal layer solution $(u(x, \epsilon), v(x, \epsilon))$. For the NF model, Nishiura and Fujii proved the following lemma.

Lemma A. The critical eigenvalues, $\mu=\sum \epsilon^{j} \mu_{j}, \mu_{0}=0$, of the operator $\epsilon^{2} D_{x x}+$ $f_{u}$, in a suitable function space, is not equal to the critical eigenvalues of (1.10), (1.11). More precisely, $\lambda_{1} \neq \mu_{1}$.

By Lemma A, $U$ can be solved from (1.10) and substituted into (1.11). This yields the reduced eigenvalue problem

$$
\lambda V=V_{x x}+g_{u}\left(\lambda-\epsilon^{2} D_{x x}-f_{u}\right)^{-1} f_{v} V+g_{v} V .
$$

The SLEP matrix is derived from the above by correctly taking the limit as $\epsilon \rightarrow 0$, which retains the contribution of the internal layers as jumps of $V_{x}$ across the layers. $\lambda_{1}<0$ is proved by studying the eigenvalues of the inverse of the SLEP matrix,

The SLEP method is an important contribution to the theory of internal layer solutions. It can be used on many systems where Lemma A is satisfied. However, it is shown by the two examples in $\S 6.2$ that Lemma A may not be satisfied for some singularly perturbed systems. The asymptotic method used in this paper does not rely on Lemma A. Assuming that the critical eigenvalues and eigenfunctions have expansions $\sum \epsilon^{j} \lambda_{j}$ and $\left(\sum \epsilon^{j} U_{j}, \sum \epsilon^{j} V_{j}\right)$, we can use the method of matched asymptotic expansion to compute $\left(\lambda_{j}, U_{j}, V_{j}\right)$ to any desired integer $j \geq 0$.

We show that the calculation of $\lambda_{j}$ for every $j>0$ is related to an $r \times r$ matrix $A$, which is not derived by the SLEP method, but is identical to the SLEP matrix [40, 38, 46]. The matrix $A$ will be called the coupling matrix, for its non-diagonal terms reflect the interaction between internal layers.

When $A$ is diagonal, the coupling is trivial and the critical eigenvalues can be determined layer by layer. An example is the original AMP model (1.3) where the 
non-coupling is due to the condition $g=0$. General conditions for the non-coupling of critical eigenvalues are obtained in this paper, cf. $\S 6.1$.

In $\S 5$, we study mono-layer solutions. A geometric method which is equivalent to, but more convenient than the shooting method is presented. We show that the mono-layer solutions are determined by the transversal intersection of a fast jump surface $\Gamma^{1}$ and a slow switching curve $\mathcal{C}$, where $\Gamma^{1}$ is the set of all points in the space of slow variables that allow a heteroclinic connection between the two slow manifolds, and $\mathcal{C}$ is the set of all points $(v, w, x), w=v_{x}$, where $h$ must switch from $h_{-}(v)$ to $h_{+}(v)$ in the slow equation (1.6), so that the mono-layer solution can satisfy boundary conditions at $x=0,1$.

It is shown in this paper that the critical eigenvalue of the mono-layer solution depends on the angle of intersection between $\Gamma^{1}$ and $\mathcal{C}$.

We comment that the condition $\mathcal{C} \pitchfork \Gamma^{1}$ is equivalent to the well-known condition on the transversal intersection of unstable and stable manifolds in the geometric singular perturbation theory. It serves as a reduction of the condition to the lower dimensional slow manifold. Details are given in $\S 5$.

In $\S 6$, we present three examples. The first example treats the AMP model where we show that $\lambda_{1}$ can be determined layer by layer and is proportional to $a^{\prime}\left(x^{i}\right)$ where $a\left(x^{i}\right)=0$ and $x^{i}$ 's are the locations of internal layers. The second example is a coupled Ginzburg-Landau equation where Lemma A is not satisfied. However, $\lambda_{1}$ can be obtained by the asymptotic method in this paper. The third example is the NF model perturbed by a fast oscillatory term $\frac{k}{\omega} \sin (\omega x+b)$. We show that the system can have any specified number of mono-layer solutions by choosing $(k, b, \omega)$. All these solutions are structurally stable regardless if some violate the Transversality Hypothesis as stated in this section. The sign of $\lambda_{1}$ may be negative or positive, depending on the angle of intersection between $\mathcal{C}$ and $\Gamma^{1}$. As the angle of intersection of $\mathcal{C}$ and $\Gamma^{1}$ goes to zero, $\lambda_{1} \rightarrow 0$. In a separate paper [18], Hale and Lin showed that the intersection of $\mathcal{C}$ and $\Gamma^{1}$ can undergo saddlenode or cusp bifurcation from their tangential intersections, which correspond to the bifurcation of mono-internal layer solutions.

In $\S 7$, we show that the method of pseudo Poincaré mappings works if the coupling matrix is nonsingular. We also discuss the stability of internal layer solutions. In particular, Theorem 7.2 solves a resolvent problem by the asymptotic method and Theorem 7.3 indicates why the stability of internal layer solutions is determined by the formal critical eigenvalues.

Our original plan was to discuss formal series only. It was suggested by the referee to outline the proofs of the existence of exact internal layer solutions and critical eigenvalue-eigenfunctions. This is done in the Appendix. We apologize for having skipped many details to keep the appendix "short"; some missing details can be found in [18]. By truncating formal series, we first obtain approximations of the exact solutions. The existence of exact layer solutions and critical eigenvalueeigenfunctions near the approximations is proved by the contraction mapping principle to the nonlinear systems, and an iteration method to the linearized systems.

Although the asymptotic method has a long history in mathematics, a systematic treatment of matched asymptotic expansions of internal layer solutions is possible only recently due to some new developments of fundamental concepts from dynamical systems theory, such as exponential dichotomies [7, 44, 45], invariant manifolds 10] and homoclinic/heteroclinic bifurcations [6, 42, 49]. Some recent works are 
listed in the Bibliography, which is far from being complete. In the past, the asymptotic method was generally regarded as non-rigorous. Recent progress in the theory of internal layer solutions has changed this greatly. Under very general conditions, it is known that there is an exact internal layer solution near the asymptotic one. The exact solution and the critical eigenvalues can be constructed by analytical methods as in [12, 29, 30, 18. Alternatively, a geometric approach based on the "Exchange Lemma" has been developed recently that can be used to prove the existence of internal layer solutions [25, 48, 27, 17, and perhaps the existence of critical eigenvalues and eigenfunctions with some modification.

\section{Notations AND BASIC LEMmas}

Assume that an internal layer solution $u(x, \epsilon)$ has $r$ internal layers near $x=$ $x_{0}^{i}, 1 \leq i \leq r$, and two boundary layers near $x^{0}=0, x^{r+1}=1$. Both internal and boundary layers are called singular layers where $u(x, \epsilon)$ does not converge uniformly to its limit as $\epsilon \rightarrow 0$. Regions that are not singular are called regular layers. We use $S$ or $R$ to denote singular or regular layers. We use $R^{i}$ or $S^{i}$ to denote the $i$ th regular or singular region. Superscripts on a solution are used to show the type of layer where the solution is located.

$$
\begin{array}{llrl}
u^{R i}(x, \epsilon) & =u(x, \epsilon), & & \text { for } x \in R^{i}, \\
u^{S i}(\xi, \epsilon) & =u\left(\epsilon \xi+x_{0}^{i}, \epsilon\right), & & \text { for } x \in S^{i} .
\end{array}
$$

Each layer is further expanded in powers of $\epsilon, u^{R i}(x, \epsilon)=\sum_{j=0}^{\infty} \epsilon^{j} u_{j}^{R i}(x)$, etc. If the type of layer is clear from the context, we sometimes drop the superscript for simplicity.

Let $C_{b u}\left(I, \mathbb{R}^{n}\right)$ be the Banach space of uniformly continuous and bounded functions with super norms. Here $I$ is a closed finite or infinite interval. Let $C_{b u}^{m}=$ $\left\{u \mid u, u^{\prime}, \ldots, u^{(m)} \in C_{b u}\right\}$ with the norm

$$
\|u\|_{C_{b u}^{m}}=\sum_{i=0}^{m}\left\|u^{(i)}\right\|_{C_{b u}} .
$$

The space $C_{b u}^{m}, m \geq 1$, is dense in $C_{b u}^{m-1}$.

For a continuous function $w(\xi)>0$, let $E_{\mathbb{R}}(w)$ be the Banach space of functions with the weight $w(\xi)$.

$$
\begin{gathered}
E_{\mathbb{R}}(w)=\left\{u: \mathbb{R} \rightarrow \mathbb{R}^{n} \mid u(\cdot) / w(\cdot) \in C_{b u}\left(\mathbb{R}, \mathbb{R}^{n}\right)\right\} . \\
\|u\|_{E(w)}=\sup \{|u(\xi) / w(\xi)|, \xi \in \mathbb{R}\} . \\
E_{\mathbb{R}}^{m}(w)=\left\{u \mid u, \ldots, u^{(m)} \in E(w)\right\} . \\
\|u\|_{E^{m}(w)}=\sum_{j=0}^{m}\left\|u^{(j)}\right\|_{E(w)} .
\end{gathered}
$$

Similarly, $E_{\mathbb{R}^{+}}^{m}(w)$ and $E_{\mathbb{R}^{-}}^{m}(w)$ are Banach spaces of weighted functions that are defined on $\mathbb{R}^{+}$and $\mathbb{R}^{-}$. We use $E^{m}(w)$ to denote $E_{\mathbb{R}}^{m}(w)$ or $E_{\mathbb{R}^{ \pm}}^{m}(w)$ if no confusion should arise. One of the most often used weights is

$$
w(\xi)=\left(1+|\xi|^{j}\right) e^{-\gamma \xi}, \quad \gamma \in \mathbb{R}, j \geq 0 .
$$

The second order equation (2.2) is equivalent to the first order system (2.3).

$$
u_{\xi \xi}+f(u)=0, \quad u \in \mathbb{R}^{n} .
$$




$$
\begin{aligned}
& u_{\xi}=v, \\
& v_{\xi}=-f(u), \quad u, v \in \mathbb{R}^{n} .
\end{aligned}
$$

Therefore the phase space for $(2.2)$ is $\mathbb{R}^{2 n}$, consisting of points $\left(u, u_{\xi}\right)$. We say $p$ is a hyperbolic equilibrium for (2.2) if $(p, 0)$ is a hyperbolic equilibrium for (2.3). We say equation

$$
u_{\xi \xi}+A(\xi) u=0
$$

has an exponential dichotomy on an interval $I \subset \mathbb{R}$ if the system

$$
\begin{aligned}
& u_{\xi}=v, \\
& v_{\xi}=-A(\xi) u
\end{aligned}
$$

has an exponential dichotomy on $I$. Here $A(\cdot): I \rightarrow \mathbb{R}^{n \times n}$ is a continuous matrix valued function. The stable and unstable subspaces and the projections associated to such spaces of (2.4) are the ones associated to that of (2.5). See [7] for an introduction of the theory of exponential dichotomies.

We say $u(\xi)$ is a heteroclinic solution of (2.2) if $\left(u(\xi), u_{\xi}(\xi)\right)$ is a heteroclinic solution for the equivalent system (2.3).

The following lemmas can be found in [33].

Lemma 2.1. Assume that $p \in \mathbb{R}^{n}, f: \mathbb{R}^{n} \rightarrow \mathbb{R}^{n}$ is $C^{1}$ and there exists $\sigma_{0}>0$

$$
f(p)=0, \operatorname{Re}\{\sigma D f(p)\} \leq-\sigma_{0} .
$$

Then

$$
u_{\xi \xi}+D f(p) u=0
$$

has an exponential dichotomy on $\mathbb{R}$ with $n$-dimensional stable and unstable spaces. Let $0<\alpha<\sqrt{\sigma_{0}}$. Then the decay rate of solutions on the stable (or unstable) subspace is bounded by $e^{-\alpha \xi}, \xi \geq 0$ (or $e^{\alpha \xi}, \xi \leq 0$ ), respectively.

Let $W^{s}$ and $W^{u}$ denote the stable and unstable subspaces of 2.7), Then $W^{s}$ and $W^{u}$ are transversal to the subspace $\{(u, v): v=0\}$, i.e.,

$$
\begin{gathered}
(u, v) \in W^{s} \cap\{v=0\} \Rightarrow u=0, \\
(u, v) \in W^{u} \cap\{v=0\} \Rightarrow u=0 .
\end{gathered}
$$

Let $p^{i}, i=1,2$, satisfy (2.6). Let $q(\xi)$ be a solution to (2.2) defined on $\mathbb{R}^{-}$with $q(\xi) \rightarrow p^{1}$ as $\xi \rightarrow-\infty$, or defined on $\mathbb{R}^{+}$with $q(\xi) \rightarrow p^{2}$ as $\xi \rightarrow \infty$. Then

$$
u_{\xi \xi}+D f(q(\xi)) u=0
$$

has exponential dichotomies on $\mathbb{R}^{-}$or $\mathbb{R}^{+}$respectively, with $\mathcal{R} P_{s}(t)$ and $\mathcal{R} P_{u}(t)$ being $n$-dimensional subspaces in $\mathbb{R}^{2 n}$. Here $P_{u}(t)+P_{s}(t)=I, t \in \mathbb{R}^{-}$or $t \in \mathbb{R}^{+}$, are the projections to the unstable and stable subspaces. Moreover, the exponential decay rate $\alpha>0$ is the same as that of (2.7). In the case where $q$ is a heteroclinic solution connecting $p^{1}$ and $p^{2}, \mathcal{R} P_{u}\left(0^{-}\right) \cap \mathcal{R} P_{s}\left(0^{+}\right)$is at least one dimensional, containing $(\dot{q}(0), \ddot{q}(0))$.

Lemma 2.2. Assume that $p^{i}, i=1,2$, and $q(\xi)$ are as in Lemma[2.1. Assume that $u_{\xi}(0) \neq 0$ for all nontrivial bounded solutions $u$ to the equation $u_{\xi \xi}+D f(q) u=0$. Let $X$ be $E_{\mathbb{R}^{+}}^{m}(w)$ or $E_{\mathbb{R}^{-}}^{m}(w)$, and $g \in X$, where $w$ is as in (2.1) and $|\gamma|<\alpha$. Then 
there exists a unique solution $u \in E_{\mathbb{R}^{+}}^{m+2}(w)$ or $E_{\mathbb{R}^{-}}^{m+2}(w)$ to the boundary value problem

$$
\begin{aligned}
& u_{\xi \xi}+D f(q) u=g, \\
& u_{\xi}(0)=\phi,
\end{aligned}
$$

Moreover,

$$
\|u\|_{E^{m+2}(w)} \leq C\left(\|g\|_{E^{m}(w)}+\|\phi\|_{\mathbb{R}^{n}}\right) .
$$

Let $p^{i} \in \mathbb{R}^{n}, i=1,2$, satisfy 2.6 . Let $q(\xi)$ be a heteroclinic solution to (2.2) connecting $p^{1}$ to $p^{2}$. Let $X=E_{\mathbb{R}}^{m}(w)$ where $w(\xi)$ is as in Lemma 2.2. Define $L_{q}: X \rightarrow X$ with $D\left(L_{q}\right)=E_{\mathbb{R}}^{m+2}(w)$ by

$$
L_{q} u=u_{\xi \xi}+D f(q(\xi)) u \text {. }
$$

Lemma 2.3. $L_{q}$ is a Fredholm operator with Fredholm index zero. Assume that $\operatorname{dim} \operatorname{Ker}\left(L_{q}\right)=1$, then $\operatorname{Ker}\left(L_{q}\right)=\operatorname{span}\{\dot{q}\}$ and Range $\left(L_{q}\right)=\{\psi\}^{\perp}$. Here $\psi$ is the unique nontrivial bounded solution for the adjoint equation, up to a scalar multiple,

$$
\begin{gathered}
L_{q}^{*} \psi \stackrel{\text { def }}{=} \psi_{\xi \xi}+D f^{\tau}(q(\xi)) \psi=0 . \\
\{\psi\}^{\perp} \stackrel{\text { def }}{=}\left\{u \in X \mid \int_{-\infty}^{\infty} \psi^{\tau}(\xi) u(\xi) d \xi=0\right\} .
\end{gathered}
$$

From Lemma 2.3, if $g \in E_{\mathbb{R}}^{m}(w)$, then equation

$$
u_{\xi \xi}+D f(q(\xi)) u=g
$$

has a solution $|u| \leq C\left(\left(1+|\xi|^{j}\right) e^{-\gamma \xi}\right)$ if and only if

$$
\int_{-\infty}^{\infty} \psi^{\tau}(\xi) g(\xi) d \xi=0
$$

Lemma 2.4. Assume the same conditions of Lemma 2.3. Let $\Psi=(-\dot{\psi}, \psi)^{\tau}$ where $\psi$ is as in Lemma [2.3. Let $\xi_{1}<0<\xi_{2}, g$ be continuous on $\left[\xi_{1}, \xi_{2}\right]$, and $\phi_{s} \in$ $\mathcal{R} P_{s}\left(\xi_{1}\right), \phi_{u} \in \mathcal{R} P_{u}\left(\xi_{2}\right)$ be two given vectors. Consider (2.10) on $\left[\xi_{1}, \xi_{2}\right]$ with the boundary conditions

$$
P_{s}\left(\xi_{1}\right)\left(\begin{array}{l}
u \\
v
\end{array}\right)=\phi_{s}, \quad P_{u}\left(\xi_{2}\right)\left(\begin{array}{l}
u \\
v
\end{array}\right)=\phi_{u} .
$$

The boundary value problem has a solution in $\left[\xi_{1}, \xi_{2}\right]$ if and only if

$$
\Psi^{\tau}\left(\xi_{1}\right) \phi_{s}\left(\xi_{1}\right)-\Psi^{\tau}\left(\xi_{2}\right) \phi_{2}\left(\xi_{2}\right)+\int_{\xi_{1}}^{\xi_{2}} \psi^{\tau}(\xi) g(\xi) d \xi=0
$$

If also $\langle\dot{q}, u\rangle+\langle\ddot{q}, v\rangle=0$, then the solution is unique and satisfies

$$
|u| \leq C\left(\left|\phi_{s}\right|+\left|\phi_{u}\right|+|g|\right),
$$

where $C$ does not depend on $\xi_{1}$ or $\xi_{2}$. 


\section{Constructing multi-layered SOlutions}

3.1. Assumptions and the existence of singular internal layer solutions. We first state the assumptions used to construct a structurally stable singular internal layer solution. It turns out that these conditions also allow us to compute all the higher order expansions of the internal layer solutions, as well as a true internal layer solution for $\epsilon>0$. Since our work relies on the normal hyperbolicity of the slow manifold, we will avoid turning points. Vector fields that satisfy our assumptions form an open set in suitable function spaces. The internal layer solutions are structurally stable in the sense that they persist when the vector fields are in that open set.

Consider a singularly perturbed system:

$$
\begin{aligned}
& u_{t}=\epsilon^{2} u_{x x}+f(u, v), \quad 0<x<1, \\
& v_{t}=v_{x x}+g(u, v), \quad u \in \mathbb{R}^{m}, v \in \mathbb{R}^{n}, \\
& u_{x}(0)=u_{x}(1)=0, \\
& A_{j} v_{x}(j)+B_{j} v(j)=\beta_{j}, \quad j=0,1 .
\end{aligned}
$$

The boundary conditions on $v$ are the Robin type. $A_{j}$ and $B_{j}$ are $n \times n$ diagonal matrices satisfying $A_{0} B_{0} \leq 0, A_{1} B_{1} \geq 0$ and $A_{j}^{2}+B_{j}^{2}=I$.

We consider stationary internal layer layer solutions of (3.1) that satisfy

$$
\begin{aligned}
& 0=\epsilon^{2} u_{x x}+f(u, v), \quad 0<x<1, \\
& u_{x}(0)=u_{x}(1)=0, \\
& 0=v_{x x}+g(u, v), \\
& A_{j} v_{x}(j)+B_{j} v(j)=\beta_{j}, \quad j=0,1 .
\end{aligned}
$$

The slow equation is often written as a first order system

$$
\begin{aligned}
v_{x} & =w, \\
w_{x} & =-g(u, v), \quad u \in \mathbb{R}^{m}, v \in \mathbb{R}^{n} .
\end{aligned}
$$

In regular layers, the $\epsilon^{2} u_{x x}$ term in (3.2) drops. The $u$-equation is algebraic. Assume

(H1) $f(u, v)=0$ has several solution manifolds $u=h^{i}(v), 0 \leq i \leq r$, on which we have

$$
\operatorname{Re}\left\{\sigma f_{u}\left(h^{i}(v), v\right)\right\}<0, \quad 0 \leq i \leq r .
$$

Condition (H1) is natural since with $v$ as a parameter, we want the reaction equation, which is an ODE, to have multiple asymptotically stable equilibria. This is related to the initial formation of internal layer solutions. See Fife [12] for further details.

With $u=h^{i}(v)$, the $v$-variable satisfies the following boundary value problem,

$$
\begin{array}{cl}
v_{x x}+g(h(v), v)=0, & 0<x<1, \\
A_{j} v_{x}(j)+B_{j} v(j)=\beta_{j}, & j=0,1,
\end{array}
$$

where $h(v)=h^{i}(v), 0 \leq i \leq r$. There are $r+1$ vector fields for the $v$-equation, each is related to one $h^{i}$. We need to find a sequence of points $\left\{x_{0}^{i}\right\}_{i=1}^{r}$ such that the switching from the $(i-1)$ th vector field to the $i$ th occurs at $x=x_{0}^{i}$. We will discuss how to find these points in detail. 
At the point of switching, the $u$ equation has a rapid jump from $u=h^{i-1}(v)$ to $h^{i}(v)$. This is possible if the second order equation $\epsilon^{2} u_{x x}+f(u, v)=0$, using a stretched variable $\xi=\left(x-x_{0}^{i}\right) / \epsilon$, has a heteroclinic solution in the following sense:

The second order equation $u_{\xi \xi}+f(u, v)=0$ can be written as a first order system $u_{\xi}=\hat{u}, \hat{u}_{\xi}=-f(u, v)$ where $v$ is a parameter. From (H1), the system has hyperbolic equilibria $u=h^{i}(v)$. The $u$-component of a heteroclinic solution to the first order system will be called a heteroclinic solution to the original second order equation. Assume the generic intersection of the unstable fibers of the equilibrium $u=h^{i-1}(v)$ and the stable fibers of the equilibrium $u=h^{i}(v)$. That is, there is a smooth $(n-1)$-dimensional surface $\Sigma^{i}$ in $\mathbb{R}^{n}$ such that the intersection is nonempty if and only if $v \in \Sigma^{i}$ and the connection breaks transversely if $v$ moves away from $\Sigma^{i}$. More precisely, assume

(H2) For any $\bar{v}^{i} \in \Sigma^{i}$, the following equation

$$
u_{\xi \xi}+f\left(u, \bar{v}^{i}\right)=0
$$

has a heteroclinic solution $q^{i}(\xi), 1 \leq i \leq r$, connecting $h^{i-1}\left(\bar{v}^{i}\right)$ to $h^{i}\left(\bar{v}^{i}\right)$. Moreover, there exists $\gamma_{0}>0$ such that, in the region $\operatorname{Re} \lambda>-\gamma_{0}$, the only eigenvalue of the linear operator on $U$

$$
U_{\xi \xi}+f_{u}\left(q^{i}(\xi), \bar{v}^{i}\right) U, \quad U \in L^{2}(\mathbb{R}),
$$

is the simple eigenvalue $\lambda=0$.

Assumption (H2) implies that the eigenspace is spanned by $\dot{q}^{i}(\xi)$, and there is a unique bounded solution $\psi^{i}, 1 \leq i \leq r$, to the adjoint equation, (see [42])

$$
\psi_{\xi \xi}+f_{u}^{\tau}\left(q^{i}(\xi), \bar{v}^{i}\right) \psi=0, \quad\left\langle\psi^{i}, \dot{q}^{i}\right\rangle=1
$$

The function $\psi^{i}$ can be used to measure the gap between the unstable fibers of $u=h^{i-1}(v)$ and the stable fibers of $u=h^{i}(v)$.

(H3) The following vector

$$
\mathbf{n}^{i}=\int_{-\infty}^{\infty} f_{v}^{\tau}\left(q^{i}(\xi), \bar{v}^{i}\right) \psi^{i}(\xi) d \xi
$$

is nonzero.

Using Melnikov's method, from (H2) and (H3), we can show that locally there exists an $(n-1)$-dimensional surface $\Sigma_{\text {local }}^{i} \subset \mathbb{R}^{n}$ for $1 \leq i \leq r$, containing $\bar{v}^{i}$, such that if $v \in \Sigma_{\text {local }}^{i}$, then $u_{\xi \xi}+f(u, v)=0$ has a heteroclinic solution $u$ near $q^{i}$. The vector $\mathbf{n}^{i}$ is the normal of $\Sigma_{\text {local }}^{i}$ at $\bar{v}^{i}$. The global surface $\Sigma^{i}$ is the union of the local ones.

We now describe the solutions of (3.4) geometrically. Let

$$
\begin{aligned}
& \Gamma^{i}=\left\{(v, w, x) \in \mathbb{R}^{n} \times \mathbb{R}^{n} \times \mathbb{R}, v \in \Sigma^{i}\right\}, \quad 1 \leq i \leq r, \\
& \Gamma^{0}=\{(v, w, x): x=0\}, \\
& \Gamma^{r+1}=\{(v, w, x): x=1\}, \\
& \mathcal{S}_{0}=\left\{(v, w, x): A_{0} w+B_{0} v=\beta_{0}, x=0\right\}, \\
& \mathcal{S}_{1}=\left\{(v, w, x): A_{1} w+B_{1} v=\beta_{1}, x=1\right\} .
\end{aligned}
$$




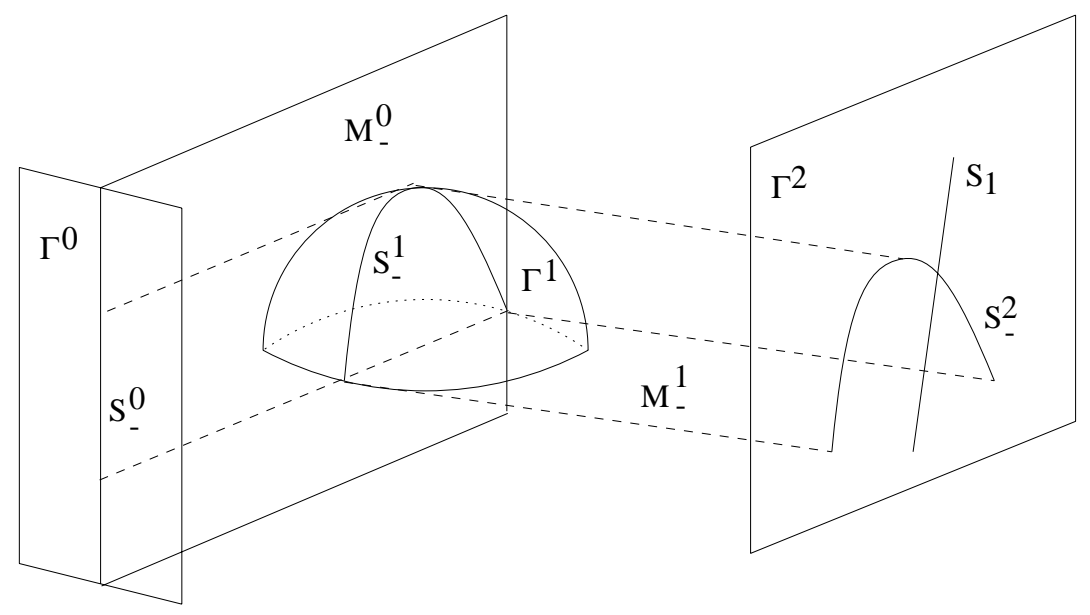

Figure 3.1. The pseudo-Poincaré mappings

Let $w=v_{x}$. Obviously $\left(v_{0}^{R}, w_{0}^{R}, x\right)$ satisfies a first order system:

$$
\begin{aligned}
& \frac{d v}{d t}=w, \\
& \frac{d w}{d t}=-g\left(h^{i}(v), v\right), \quad 0 \leq i \leq r, \\
& \frac{d x}{d t}=1 .
\end{aligned}
$$

Because of the boundary conditions, $(v, w, x)$ must start at $\mathcal{S}_{0}$ and end at $\mathcal{S}_{1}$ and switch from $u=h^{i-1}(v)$ to $h^{i}(v)$ at each switching point $\wp^{i} \stackrel{\text { def }}{=}\left(v_{0}^{R}\left(x_{0}^{i}\right), w_{0}^{R}\left(x_{0}^{i}\right), x_{0}^{i}\right)$ on $\Gamma^{i}$

Additional knowledge regarding the flow on the slow manifold and $\Gamma^{i}$ is needed in order to form the solution of (3.6). In [30], it is assumed that the flow of (3.6) is transverse to each $\Gamma^{i}$. Then a Poincaré mapping $\mathcal{P}^{i}: \Gamma^{i} \rightarrow \Gamma^{i+1}, 0 \leq i \leq r$, can be defined. A shooting method can be used to find the switching points. However, as mentioned in the introduction, the transversality condition is too strong so that it rules out some structurally stable internal layer solutions.

To use a shooting method without assuming the transversality of the slow flow to $\Gamma^{i}$, define the pseudo-Poincaré mappings as follows:

For the $i$ th vector field, let the solution of (3.6) be $\Phi^{i}(x)$. Let $\mathcal{S}_{-}^{0}=\mathcal{S}_{0}$. Since the flow $\Phi^{0}(\cdot)$ is transverse to $\mathcal{S}_{-}^{0}, \mathcal{M}_{-}^{0} \stackrel{\text { def }}{=} \bigcup\left\{\Phi^{0}(x) \cdot \mathcal{S}_{-}^{0}, x \geq 0\right\}$ is an $(n+1)$ dimensional smooth manifold. Assume that $\mathcal{M}_{-}^{0}$ intersects $\Gamma^{1}$ transversely. $\mathcal{S}_{-}^{1} \stackrel{\text { def }}{=}$ $\mathcal{M}_{-}^{0} \pitchfork \Gamma^{1}$ is an $n$-dimensional submanifold of $\Gamma^{1}$. The procedure of associating the sets $\mathcal{S}_{-}^{0} \rightarrow \mathcal{S}_{-}^{1}$, denoted by $\mathcal{P}^{0}$, is a mapping between two sets. The mapping $\mathcal{P}^{0}$ will be called a pseudo-Poincaré mapping. See Figure 3.1.

We now proceed inductively. Assume that an $n$-dimensional submanifold $\mathcal{S}_{-}^{i} \subset$ $\Gamma^{i}$ has been defined. Assume that:

(H4) The flow $\Phi^{i}(\cdot)$ is transverse to $\mathcal{S}_{-}^{i}, 1 \leq i \leq r$. 
Assumption (H4) implies that

$$
\mathcal{M}_{-}^{i} \stackrel{\text { def }}{=} \bigcup\left\{\Phi^{i}(x) \cdot \mathcal{S}_{-}^{i}, x \geq 0\right\}
$$

is a smooth $(n+1)$-dimensional manifold. Assume that:

(H5) $\mathcal{M}_{-}^{i} \pitchfork \Gamma^{i+1}$.

Assumption (H5) implies that

$$
\mathcal{S}_{-}^{i+1} \stackrel{\text { def }}{=} \mathcal{M}_{-}^{i} \cap \Gamma^{i+1} .
$$

is an $n$-dimensional smooth submanifold of $\Gamma^{i+1}$. With (H4) and (H5), the pseudoPoincaré mapping

$$
\mathcal{P}^{i}: \mathcal{S}_{-}^{i} \rightarrow \mathcal{S}_{-}^{i+1}
$$

is locally uniquely defined. We assume that:

(H6) The image of $\mathcal{S}_{-}^{0}$ under the composite mapping:

$$
\mathcal{P}^{r} \ldots \mathcal{P}^{1} \cdot \mathcal{P}^{0}
$$

intersects transversely and nonemptily with $\mathcal{S}_{1}$ in $\Gamma^{r+1}$.

The intersection clearly determines $\left(v_{0}^{R}(1), w_{0}^{R}(1)\right)$ locally uniquely. The pseudoPoincaré mapping is only a set mapping, but due to (H4), its inverse is a smooth point mapping form $\mathcal{S}_{-}^{i+1} \rightarrow \mathcal{S}_{-}^{i}$. The switching points $\wp^{i}$ on the surfaces $\Gamma^{i}$ can be obtained by applying the inverse mappings of $\mathcal{P}^{r}, \cdots, \mathcal{P}^{0}$ to $\mathcal{S}_{-}^{r+1} \cap \mathcal{S}_{1}$ successively. The solution $\left(v_{0}^{R}, w_{0}^{R}, x\right)$ of (3.6) can be computed by using $\Phi^{i}(x)$ between these switching points.

Remark. Similar to the construction of $\mathcal{P}^{i}$ but going backwards in time, we can define pseudo-Poincaré mappings $\mathcal{Q}^{i}: \mathcal{S}_{+}^{i+1} \rightarrow \mathcal{S}_{+}^{i}, r \geq i \geq 0$, starting with $\mathcal{S}_{+}^{r+1}=$ $\mathcal{S}_{1}$. However, this time we do not need any new assumptions. It can be shown that $\mathcal{Q}^{i}$ is well defined by the same assumptions (H4)-(H6). Also, on each $\Gamma^{i}$, we can show that $\mathcal{S}_{-}^{i} \pitchfork \mathcal{S}_{+}^{i}$, which uniquely determines the switching point $\wp^{i}$. These comments are of theoretical interest but will not be used in the sequel. The proof is omitted.

We have found a sequence of points

$$
x_{0}^{0}=0<x_{0}^{1}<x_{0}^{2}<\cdots<x_{0}^{r}<1=x_{0}^{r+1}
$$

and a solution $v_{0}^{R} \in C^{1}([0,1])$ of (3.4). The second derivative $v_{x x}$ may have a jump across $x_{0}^{i}$. The solution $v_{0}^{R}$ is piecewise $C^{\infty}$ on $\left(x_{0}^{i}, x_{0}^{i+1}\right)$ and is in $C^{\infty}\left(\left[x_{0}^{i}, x_{0}^{i+1}\right]\right)$ by choosing one sided limits at $x_{0}^{i}$ and $x_{0}^{i+1}$.

Let $q^{0}=h^{0}\left(v_{0}^{R}(0)\right), \bar{v}^{0}=v_{0}^{R}(0), q^{r+1}=h^{r}\left(v_{0}^{R}(1)\right), \bar{v}^{r+1}=v_{0}^{R}(1)$ be constant functions.

The union of the regular and singular (boundary and internal) solutions,

$$
\begin{aligned}
& u_{0}^{R}=h^{i}\left(v_{0}^{R}(x)\right), v_{0}^{R}=v_{0}^{R}(x), \text { for } x_{0}^{i} \leq x \leq x_{0}^{i+1}, 0 \leq i \leq r, \\
& u_{0}^{S i}=q^{i}(\xi), v_{0}^{S i}=\bar{v}^{i}, \quad \begin{cases}\text { for } \xi \in \mathbb{R}, & 1 \leq i \leq r, \\
\text { for } \xi \in \mathbb{R}^{+}, & i=0, \\
\text { for } \xi \in \mathbb{R}^{-}, & i=r+1,\end{cases}
\end{aligned}
$$

forms a singular internal layer solution. It is the 0th order expansion for a multiple internal layer solution. 
Based on the singular internal layer solutions, in the Appendix we show by Newton's method that for small $\epsilon>0$, there is a unique true multiple internal layer solution that is near the singular internal layer solution, and admits asymptotic expansions to any orders in $\epsilon$. See [12 [30, 18]. The existence has also been proved by a geometric method [25, 48]. The rest of this section is devoted to computing the expansions of $(u(\epsilon), v(\epsilon))$.

3.2. Formal expansions of the layered solutions. Denote the expansion in regular layers by

$$
\left(u^{R}(x, \epsilon), v^{R}(x, \epsilon)\right)=\left(\sum_{0}^{\infty} \epsilon^{j} u_{j}^{R}(x), \sum_{0}^{\infty} \epsilon^{j} v_{j}^{R}(x)\right) .
$$

Let the position of the internal and boundary layers be $x^{i}(\epsilon)$ and introduce a stretched variable $\xi$ near $x^{i}(\epsilon)$.

$$
\begin{aligned}
x^{i}(\epsilon) & =\sum_{0}^{\infty} \epsilon^{j} x_{j}^{i}, \quad 0 \leq i \leq r+1, \\
\xi & =\left(x-x^{i}(\epsilon)\right) / \epsilon,
\end{aligned}
$$

where $\xi \in \mathbb{R}$ for $1 \leq i \leq r, \xi \geq 0$ for $i=0$ and $\xi \leq 0$ for $i=r+1$. The leading term $x_{0}^{i}$ has been obtained by the shooting method. Since the position of the boundary layers are $\epsilon$ independent, let $x_{j}^{0}=x_{j}^{r+1}=0$ for all $j \geq 1$. Denote the expansion of boundary and internal layers near $x^{i}(\epsilon)$ by

$$
\left(u^{S i}(\xi, \epsilon), v^{S i}(\xi, \epsilon)\right)=\left(\sum_{0}^{\infty} \epsilon^{j} u_{j}^{S i}(\xi), \sum_{0}^{\infty} \epsilon^{j} v_{j}^{S i}(\xi)\right) .
$$

The expansions are determined by three things. They must formally satisfy differential equations derived from (3.2) and (3.3), boundary conditions in boundary layers, and matching conditions between any two adjacent regular and singular layers. We now describe them in detail.

1. In regular layers, the expansions must satisfy

$$
0=\epsilon^{2} u_{x x}^{R}+f\left(u^{R}, v^{R}\right), \quad 0=v_{x x}^{R}+g\left(u^{R}, v^{R}\right) .
$$

In singular (boundary and internal) layers, using $\xi=\left(x-x^{i}(\epsilon)\right) / \epsilon$,

$$
\begin{gathered}
0=u_{\xi \xi}^{S}+f\left(u^{S}, v^{S}\right), \quad 0=v_{\xi \xi}^{S}+\epsilon^{2} g\left(u^{S}, v^{S}\right), \quad \text { or } \\
v_{\xi}^{S}=\epsilon w^{S}, \quad w_{\xi}^{S}=-\epsilon g\left(u^{S}, v^{S}\right) .
\end{gathered}
$$

Remark. Avoid the erroneous choices $v_{\xi}^{S}=w^{S}, w_{\xi}^{S}=\epsilon^{2} g\left(u^{S}, v^{S}\right)$ and $v_{\xi}^{S}=w^{S}, w_{\xi}^{S}$ $=g\left(u^{S}, v^{S}\right)$, which we realized only after unsuccessful trials.

We shall see in the future that $v_{0}^{S}$ is a constant function. Thus, $w^{S}(\xi, \epsilon)=$ $v_{\xi}^{S}(\xi, \epsilon) / \epsilon=\sum_{j=0}^{\infty} \epsilon^{j} w_{j}^{S}(\xi)$. The formal expansion of $w^{S}$ starts from the $\epsilon^{0}$ term rather than the $\epsilon^{-1}$ term. 
2. Boundary conditions in the boundary layers.

The following boundary conditions for $\left(u^{S}(\xi, \epsilon), v^{S}(\xi, \epsilon)\right)$ are to be satisfied in boundary layers:

$$
\begin{aligned}
& u_{\xi}^{S i}(0, \epsilon)=0, \quad i=0, r+1, \\
& A_{0} w^{S 0}(0, \epsilon)+B_{0} v^{S 0}(0, \epsilon)=\beta_{0}, \\
& A_{1} w^{S, r+1}(0, \epsilon)+B_{1} v^{S, r+1}(0, \epsilon)=\beta_{1} .
\end{aligned}
$$

3. Exponential matching principles.

Let $u^{R}$ be the outer solution in one of the regular layers adjacent either to the left or right of $x_{0}^{i}$. The expansion of $u^{R}$ by the inner variable $\xi$ is denoted $\tilde{u}^{R}$.

$$
\begin{aligned}
& \sum_{0}^{\infty} \epsilon^{j} \tilde{u}_{j}^{R}(\xi)=u^{R}\left(\sum_{0}^{\infty} \epsilon^{j} x_{j}^{i}+\epsilon \xi\right), \\
& \sum_{0}^{\infty} \epsilon^{j} \tilde{v}_{j}^{R}(\xi)=v^{R}\left(\sum_{0}^{\infty} \epsilon^{j} x_{j}^{i}+\epsilon \xi\right) .
\end{aligned}
$$

\section{The exponential matching principle.}

$$
\begin{aligned}
\left|\tilde{u}_{j}^{R}(\xi)-u_{j}^{S}(\xi)\right|+\left|\tilde{u}_{j \xi}^{R}(\xi)-u_{j \xi}^{S}(\xi)\right| & \leq C\left(1+|\xi|^{j}\right) e^{-\gamma|\xi|} \\
\left|\tilde{v}_{j}^{R}(\xi)-v_{j}^{S}(\xi)\right|+\left|\tilde{v}_{j \xi}^{R}(\xi)-v_{j \xi}^{S}(\xi)\right| & \leq C\left(1+|\xi|^{j}\right) e^{-\gamma|\xi|} .
\end{aligned}
$$

Let $\sum_{0}^{\infty} \tilde{w}_{j}^{R}(\xi)$ denote the inner expansion of $w^{R}(\epsilon)$. If we recall that

$$
\begin{array}{cl}
v_{x}^{R}(\epsilon)=w^{R}(\epsilon), & \text { in regular layers, } \\
v_{\xi}^{S}(\epsilon)=\epsilon w^{S}(\epsilon), & \text { in singular layers }
\end{array}
$$

we can rewrite (3.14) in an equivalent form

$$
\left|\tilde{v}_{j}^{R}(\xi)-v_{j}^{S}(\xi)\right|+\left|\tilde{w}_{j}^{R}(\xi)-w_{j}^{S}(\xi)\right| \leq C\left(1+|\xi|^{j}\right) e^{-\gamma|\xi|} .
$$

The main result of this section is the following theorem.

Theorem 3.1. Suppose (H1)-(H6) are satisfied. Then there exists a unique formal series expansion of internal layer solution for (3.2). The solution has $r$ internal layers and two boundary layers. The formal series solution satisfies (3.8) in regular layers, and (3.9) in singular layers. Moreover, the expansions must satisfy (3.10)(3.12) in boundary layers, and the matching of adjacent regular and singular layers (3.13) and (3.14).

The 0 th order expansion $\left(u_{0}^{R}, v_{0}^{R}\right)$ in regular layers and $\left(u_{0}^{S}, v_{0}^{S}\right)$ in singular layers form a singular internal layer solution. In singular layers, $u_{0}^{S i}=q^{i}, v_{0}^{S i}=\bar{v}^{i}$ with $\bar{v}^{i} \in \Sigma^{i}$ if $1 \leq i \leq r$. In regular layers, $v_{0}^{R}$ is a $C^{1}(0,1)$ solution for (3.4) with $v_{0}^{R}\left(x_{0}^{i}\right)=\bar{v}^{i}$, and $u_{0}^{R}(x)=h^{i}\left(v_{0}^{R}(x)\right)$ if $x \in\left(x^{i}, x^{i+1}\right)$.

Higher order expansions of solutions are computable by a recursive procedure described in the rest of the section.

Recall that $[h]\left(x_{0}^{i}\right)=h\left(x_{0}^{i}+\right)-h\left(x_{0}^{i}-\right)$. We need to consider the following boundary value problem with interface conditions at $x_{0}^{i}, 1 \leq i \leq r$. 
(BVPIC)

$$
\begin{aligned}
& V_{x x}-\left(g_{u} f_{u}^{-1} f_{v}-g_{v}\right) V=E_{1}, \\
& A_{j} V_{x}(j)+B_{j} V(j)=E_{2 j}, \quad j=0,1, \\
& \mathbf{n}^{i} \cdot\left(w_{0}^{R}\left(x_{0}^{i}\right) \Delta x^{i}+V\left(x_{0}^{i}+\right)\right)=E_{3}^{i}, \\
& {[V]\left(x_{0}^{i}\right)=E_{4}^{i},} \\
& {\left[V_{x}\right]\left(x_{0}^{i}\right)+\left[w_{0 x}^{R}\right]\left(x_{0}^{i}\right) \Delta x^{i}=E_{5}^{i} .}
\end{aligned}
$$

Here $f=f\left(u_{0}^{R}, v_{0}^{R}\right), g=g\left(u_{0}^{R}, v_{0}^{R}\right), E_{1}:[0,1] \rightarrow \mathbb{R}^{n}$ is $C^{\infty}$ on each $\left(x_{0}^{i}, x_{0}^{i+1}\right)$ and has a $C^{\infty}$ extension to $\left[x_{0}^{i}, x_{0}^{i+1}\right], E_{3}^{i} \in \mathbb{R}$, and $E_{2 j}, E_{4}^{i}, E_{5}^{i} \in \mathbb{R}^{n},\left\{\Delta x^{i}\right\}_{i=1}^{r}$ is a set of parameters, to be determined as part of the solution.

Lemma 3.2. Assume that (H4)-(H6) are satisfied, then for all $\left(E_{1}, E_{2 j}, E_{3}, E_{4}, E_{5}\right)$, the $(B V P I C)$ has a unique solution $V:[0,1] \rightarrow \mathbb{R}^{n}$ and $\left\{\Delta x^{i}\right\}_{1}^{r}$. The solution $V$ is in $C^{\infty}\left(\left(x_{0}^{i}, x_{0}^{i+1}\right)\right)$ and has a $C^{\infty}$ extension on each closed interval $\left[x_{0}^{i}, x_{0}^{i+1}\right]$.

Remark. With one more condition (H7), we can show that if the SLEP matrix, introduced in $\S 4$, is nonsingular, then (BVPIC) has a unique solution. See $\S 7$.

Proof. The general solution for the first equation in (BVPIC) contains $2 n(r+1)$ parameters, that is, $2 n$ parameters in each of the $r+1$ intervals $\left(x_{0}^{i}, x_{0}^{i+1}\right)$. Together with $\Delta x^{i}, 1 \leq i \leq r$, we have $2 n(r+1)+r$ unknowns, which have to be determined from the other 4 equations in the (BVPIC). It is easy to see that the other 4 equations lead to a $[2 n(r+1)+r] \times[2 n(r+1)+r]$ linear algebraic system of equations. The Fredholm index for this finite dimensional linear system is zero. It suffices to show that if all the right hand sides are zero, then the (BVPIC) has only the trivial solution $V=0,\left\{\Delta x^{i}\right\}_{1}^{r}=0$.

The second order equation for $V$ is converted into a first order system

$$
\begin{aligned}
d V / d t & =W, \\
d W / d t & =\left(g_{u} f_{u}^{-1} f_{v}-g_{v}\right) V, \\
d x / d t & =1 .
\end{aligned}
$$

Assume the $(V, W)$ and $\left\{\Delta x^{i}\right\}_{1}^{r}$ is a solution of the homogeneous (BVPIC). Let $\Delta x^{0}=\Delta x^{r+1}=0$. We show that at the switching point $\wp^{i}$ the vectors

$\left(V\left(x_{0}^{i} \pm\right)+v_{0 x}^{R}\left(x_{0}^{i} \pm\right) \Delta x^{i}, W\left(x_{0}^{i} \pm\right)+w_{0 x}^{R}\left(x_{0}^{i} \pm\right) \Delta x^{i}, \Delta x^{i}\right) \in T_{\wp^{i}} \mathcal{S}_{-}^{i}, \quad 0 \leq i \leq r+1$,

where minus (or plus) is ignored if $i=0$ (or $i=r+1)$. Due to the interface condition, the two vectors corresponding to plus and minus are equal and denoted $\mathbf{a}^{i}$.

For $i=0$, because of $\Delta x^{0}=0$ and the boundary condition at $x=0$, it is clear that $\mathbf{a}^{0}=(V(0), W(0), 0) \in T_{\wp^{0}} \mathcal{S}_{-}^{0}$.

Assume that (3.15) is true for $i$, we show that it is true for $i+1$. First, the assumption implies that $\left(V^{i}\left(x_{0}^{i}+\right), W^{i}\left(x_{0}^{i}+\right), 0\right) \in T_{\wp^{i}} \mathcal{M}_{-}^{i}$. Applying $D \Phi^{i}$, we find that $\left(V\left(x_{0}^{i+1}-\right), W\left(x_{0}^{i+1}-\right), 0\right) \in T_{\wp^{i+1}} \mathcal{M}_{-}^{i}$. Observe that

$$
\Delta x^{i}\left(v_{0 x}^{R}\left(x_{0}^{i+1}-\right), w_{0 x}^{R}\left(x_{0}^{i+1}-\right), 1\right) \in T_{\wp^{i+1}} \mathcal{M}_{-}^{i} .
$$

Adding the two vectors, we have proved that the sum $\mathbf{a}^{i+1}$ is in $T_{\wp^{i}} \mathcal{M}_{-}^{i}$.

From the third equation of (BVPIC), $\mathbf{a}^{i+1}$ is tangent to $\Gamma^{i+1}$ at $\wp^{i+1}$. Since $T_{\wp^{i+1}} \mathcal{S}_{-}^{i+1}=T_{\wp^{i+1}} \mathcal{M}_{-}^{i} \cap T_{\wp^{i+1}} \Gamma^{i+1}$, thus $\mathbf{a}^{i+1} \in T_{\wp^{i+1}} \mathcal{S}_{-}^{i+1}$.

The statement (3.15) has been proved by induction. 
From $\Delta x^{r+1}=0$, the vector $\mathbf{a}^{r+1}$ is also in $T_{\wp^{r+1}} \mathcal{S}_{1}$. From (H6), $\mathbf{a}^{r+1}=0$. Tracing backwards in time and using (H4), we can prove that $\mathbf{a}^{i}=0$ for all $0 \leq i \leq$ $r+1$. This proves that $\left(V, W,\left\{\Delta x^{i}\right\}_{1}^{r}\right)$ is a zero solution.

Equations satisfied by $\left(u_{j}^{R}, v_{j}^{R}\right)$ and $\left(u_{j}^{S}, v_{j}^{S}\right)$ are obtained by expanding (3.8) and (3.9) in powers of $\epsilon$.

$\epsilon^{0}$ th order expansion. In regular layers:

$$
\begin{aligned}
& f(u, v)=0, \\
& v_{x x}+g(u, v)=0 .
\end{aligned}
$$

From (H1), the first equation has locally unique solutions $h(v)=h^{i}(v), 0 \leq i \leq r$. Substituting into the second equation, we have

$$
v_{x x}+g(h(v), v)=0,
$$

where $h=h^{i}$ for $x_{0}^{i}<x<x_{0}^{i+1}$. The function $v_{0}^{R}$, obtained by the shooting method, is a solution of the above. To uniquely determine $v_{0}^{R}$, we must find jumps of $\left(v_{0}^{R}, v_{0 x}^{R}\right)$ across each $x_{0}^{i}$ and the boundary conditions at $x=0,1$.

In singular layers (internal and boundary layers):

$$
\begin{aligned}
& u_{\xi \xi}+f(u, v)=0, \\
& v_{\xi}=0, \\
& w_{\xi}=0 .
\end{aligned}
$$

The above indicates that $v_{0}^{S}, w_{0}^{S}$ are constants in singular layers. Let $v_{0}^{S i}=$ $\bar{v}^{i}, w_{0}^{S i}=\bar{w}^{i}$. From the matching conditions, we have

$$
\begin{gathered}
v_{0}^{S i}=\bar{v}^{i}=v_{0}^{R}\left(x_{0}^{i}+\right)=v_{0}^{R}\left(x_{0}^{i}-\right), \\
w_{0}^{S i}=\bar{w}^{i}=w_{0}^{R}\left(x_{0}^{i}+\right)=w_{0}^{R}\left(x_{0}^{i}-\right) .
\end{gathered}
$$

In particular, $\left(v_{0}^{R}, w_{0}^{R}\right)$ has no jump throughout $[0,1]$. Thus, $v_{0}^{R} \in C^{1}([0,1])$.

By expanding (3.11), (3.12), the boundary conditions on $\left(v_{0}^{S}, w_{0}^{S}\right)$ are

$$
\begin{aligned}
& A_{0} w_{0}^{S 0}(0)+B_{0} v_{0}^{S 0}(0)=\beta_{0}, \\
& A_{1} w_{0}^{S, r+1}(0)+B_{1} v_{0}^{S, r+1}(0)=\beta_{1} .
\end{aligned}
$$

Based on the matching principle, we conclude that the boundary conditions on $\left(v_{0}^{R}, w_{0}^{R}\right)$ are precisely as in (3.4). Therefore $v_{0}^{R}$ is the unique solution to the $\epsilon^{0}$ th expansion in regular layers.

We now consider the $u$ equation in singular layers.

$$
u_{\xi \xi}+f\left(u, \bar{v}^{i}\right)=0, \quad 0 \leq i \leq r+1 .
$$

From the matching conditions, $u_{0}^{S i}$ should satisfy

$$
u_{0}^{S i}(\xi) \rightarrow \begin{cases}h^{i}\left(v_{0}^{R}\left(x_{0}^{i}\right)\right), \xi \rightarrow-\infty, & \text { if } 1 \leq i, \\ h^{i+1}\left(v_{0}^{R}\left(x_{0}^{i}\right)\right), \xi \rightarrow \infty, & \text { if } i \leq r .\end{cases}
$$

If $i=0, r+1, u_{0}^{S i}$ should also satisfy the boundary condition $u_{0 \xi}^{S i}(0)=0$. All these are satisfied if $u_{0}^{S i}=q^{i}$ in the $i$ th singular layer.

Higher order expansions. Assume that $u_{\ell}^{R}, v_{\ell}^{R}, u_{\ell}^{S}, v_{\ell}^{S}, x_{\ell}^{i}, 0 \leq \ell<j$, have been obtained. They satisfy the equations, boundary conditions and matching conditions up to $\epsilon^{j-1}$. 
In regular layers. Consider the $\epsilon^{1}$ th order expansion first,

$$
\begin{aligned}
f_{u} u_{1}+f_{v} v_{1} & =0, \\
v_{1 x x}+g_{u} u_{1}+g_{v} v_{1} & =0 .
\end{aligned}
$$

Here the arguments of $f_{u}, f_{v}, g_{u}, g_{v}$ are $\left(u_{0}^{R}, v_{0}^{R}\right)$. From (3.19) and (3.20), we have

$$
\begin{aligned}
& u_{1}=-f_{u}^{-1} f_{v} v_{1}, \\
& v_{1 x x}-\left(g_{u} f_{u}^{-1} f_{v}-g_{v}\right) v_{1}=0 .
\end{aligned}
$$

Consider the $\epsilon^{j}$ th order expansion. All the terms involving only $u_{\ell}^{R}, v_{\ell}^{R}, \ell<j$, will be denoted $\ell \cdot o \cdot t$.

$$
\begin{aligned}
u_{j-2, x x}+f_{u} u_{j}+f_{v} v_{j} & =\ell \cdot o \cdot t, \\
v_{j x x}+g_{u} u_{j}+g_{v} v_{j} & =\ell \cdot o \cdot t .
\end{aligned}
$$

Actually $u_{j-2, x x} \in \ell \cdot o \cdot t$. From (3.22) and (3.23), we have

$$
\begin{aligned}
& u_{j}=-f_{u}^{-1} f_{v} v_{j}+\ell \cdot o \cdot t, \\
& v_{j x x}-\left(g_{u} f_{u}^{-1} f_{v}-g_{v}\right) v_{j}=\ell \cdot o \cdot t .
\end{aligned}
$$

From Lemma 3.2, if we can derive boundary conditions at $x=0,1$ and interface conditions at $x_{0}^{i}, 1 \leq i \leq r$, we can uniquely solve $v_{j}^{R}$, whence $u_{j}^{R}$.

In singular layers. Consider the $\epsilon^{1}$ th order expansion first,

$$
\begin{aligned}
& u_{1 \xi \xi}+f_{u} u_{1}+f_{v} v_{1}=0, \\
& v_{1 \xi}=w_{0}^{S}=\bar{w}^{i}, \\
& w_{1 \xi}=-g\left(u_{0}^{S}, \bar{v}^{i}\right) .
\end{aligned}
$$

The arguments of $f_{u}, f_{v}, g_{u}, g_{v}$ are $\left(u_{0}^{S}, v_{0}^{S}\right)$.

If we know $v_{1}^{S}(0), w_{1}^{S}(0)$, then,

$$
\begin{aligned}
v_{1}^{S}(\xi) & =v_{1}^{S}(0)+\bar{w}^{i} \xi \\
w_{1}^{S}(\xi) & =w_{1}^{S}(0)-\int_{0}^{\xi} g\left(u_{0}^{S}, \bar{v}^{i}\right) d s .
\end{aligned}
$$

Consider the $\epsilon^{j}$ th order expansion. All the terms involving only $u_{\ell}^{S}, v_{\ell}^{S}, \ell<j$, will be denoted $\ell \cdot o \cdot t$.

$$
\begin{aligned}
& u_{j \xi \xi}+f_{u} u_{j}+f_{v} v_{j}=\ell \cdot o \cdot t, \\
& v_{j \xi}=w_{j-1}, \\
& w_{j \xi}=-g_{u} u_{j-1}-g_{v} v_{j-1}+\ell \cdot o \cdot t .
\end{aligned}
$$

If we know $v_{j}^{S}(0), w_{j}^{S}(0)$, then

$$
\begin{aligned}
& v_{j}(\xi)=v_{j}^{S}(0)+\int_{0}^{\xi} \ell \cdot o \cdot t, \\
& w_{j}(\xi)=w_{j}^{S}(0)+\int_{0}^{\xi} \ell \cdot o \cdot t .
\end{aligned}
$$

Plug (3.32) into (3.29),

$$
u_{j \xi \xi}+f_{u} u_{j}+f_{v}\left(v_{j}^{S}(0)+\ell \cdot o \cdot t\right)=\ell \cdot o \cdot t .
$$


It is easy to verify by induction that $\ell \cdot o \cdot t$ terms above satisfy

$$
|\ell \cdot o \cdot t| \leq C\left(1+|\xi|^{j}\right)
$$

Using Lemma 2.3. (3.34) has a unique solution satisfying

$$
\left|u_{j}^{S}\right| \leq C\left(1+|\xi|^{j}\right), \quad u_{j}^{S}(0) \perp \dot{q}^{i}(0),
$$

if and only if

$$
\int_{-\infty}^{\infty} \psi^{i}(\xi) \cdot f_{v} v_{j}^{S}(0) d \xi=\ell \cdot o \cdot t
$$

By the definition of $\mathbf{n}^{i}$ in (3.5), which is the normal of $\Sigma^{i}$, the condition simplifies to

$$
\mathbf{n}^{i} \cdot v_{j}^{S}(0)=\ell \cdot o \cdot t
$$

By induction also, the integrands in the right sides of (3.32) and (3.33) are of $O\left(1+|\xi|^{j-1}\right)$, therefore,

$$
\left|v_{j}^{S}\right|+\left|w_{j}^{S}\right| \leq C\left(1+|\xi|^{j}\right) .
$$

To further compute $v_{j}^{R}, w_{j}^{R}, v_{j}^{S}$ and $w_{j}^{S}$, we must use the matching conditions. Recall that $v_{j}^{R}, w_{j}^{R}$ are $C^{\infty}$ in $\left(x_{0}^{i}, x_{0}^{i+1}\right)$. The domain where the functions are $C^{\infty}$ can be extended to an open interval containing $\left[x_{0}^{i}, x_{0}^{i+1}\right]$. Consider the inner expansion of outer solutions from the right of $x_{0}^{i}$,

$$
\begin{aligned}
& \sum_{0}^{\infty} \epsilon^{j} \tilde{v}_{j}^{R}(\xi)=v^{R}\left(\sum_{0}^{\infty} \epsilon^{j} x_{j}^{i}+\epsilon \xi\right) \\
& \sum_{0}^{\infty} \epsilon^{j} \tilde{w}_{j}^{R}(\xi)=w^{R}\left(\sum_{0}^{\infty} \epsilon^{j} x_{j}^{i}+\epsilon \xi\right) .
\end{aligned}
$$

In particular,

$$
\begin{aligned}
\tilde{v}_{0}^{R}(\xi) & =v_{0}^{R}\left(x_{0}^{i}\right), \\
\tilde{w}_{0}^{R}(\xi) & =w_{0}^{R}\left(x_{0}^{i}\right), \\
\tilde{v}_{1}^{R}(\xi) & =v_{1}^{R}\left(x_{0}^{i}+\right)+v_{0 x}^{R}\left(x_{0}^{i}\right)\left(x_{1}^{i}+\xi\right), \\
\tilde{w}_{1}^{R}(\xi) & =w_{1}^{R}\left(x_{0}^{i}+\right)+w_{0 x}^{R}\left(x_{0}^{i}+\right)\left(x_{1}^{i}+\xi\right), \\
\tilde{v}_{j}^{R}(\xi) & =v_{j}^{R}\left(x_{0}^{i}+\right)+v_{0 x}^{R}\left(x_{0}^{i}\right) x_{j}^{i}+\theta_{j}+P_{j}^{v}, \\
\tilde{w}_{j}^{R}(\xi) & =w_{j}^{R}\left(x_{0}^{i}+\right)+w_{0 x}^{R}\left(x_{0}^{i}+\right) x_{j}^{i}+\delta_{j}+P_{j}^{w},
\end{aligned}
$$

The constants $\theta_{j}$ and $\delta_{j}$ depend on $x_{\ell}^{i}, 1 \leq \ell<j$. The polynomials $P_{j}^{v}, P_{j}^{w}$ contain terms $\xi^{\ell}, \ell>0$, only. Similar formulas hold for the expansions of $\left(u^{R}, v^{R}\right)$ from the left of $x_{0}^{i}$. On the surface, the matching of $v_{j}^{S}$ and $\tilde{v}_{j}^{R}$ or $w_{j}^{S}$ and $\tilde{w}_{j}^{R}$ is very complicated, since all the powers of $\xi$ have to be matched. However, we can show the following.

Lemma 3.3. For all the choices of $\left(v_{j}^{S}(0), w_{j}^{S}(0)\right)$, we have

$$
\begin{aligned}
v_{j}^{S}-\tilde{v}_{j}^{R} & =C_{1}+O\left(\left(1+|\xi|^{j}\right) e^{-\gamma|\xi|}\right), \\
w_{j}^{S}-\tilde{w}_{j}^{R} & =C_{2}+O\left(\left(1+|\xi|^{j}\right) e^{-\gamma|\xi|}\right),
\end{aligned}
$$

where the constants $C_{1}, C_{2}$ depend on $\left(v_{j}^{S}(0), w_{j}^{S}(0)\right)$. 
Proof. Recall that $\left(\sum \epsilon^{j} v_{j}^{R}, \sum \epsilon^{j} w_{j}^{R}\right)$ satisfies (3.3). Using the stretched variable $\xi=\left(x-\sum \epsilon^{j} x_{j}^{i}\right) / \epsilon$, we find that $\left(\sum \epsilon^{j} \tilde{v}_{j}^{R}, \sum \epsilon^{j} \tilde{w}_{j}^{R}\right)$ formally satisfies

$$
\begin{aligned}
\tilde{v}_{\xi}^{R} & =\epsilon \tilde{w}^{R}, \\
\tilde{w}_{\xi}^{R} & =-\epsilon g\left(\tilde{u}^{R}, \tilde{v}^{R}\right) .
\end{aligned}
$$

Expanding in the powers of $\epsilon$, we find that $\left(\tilde{v}_{j}^{R}, \tilde{w}_{j}^{R}\right)$ satisfies equations similar to (3.30), (3.31).

$$
\begin{aligned}
\tilde{v}_{j \xi}^{R} & =\tilde{w}_{j-1}^{R}, \\
\tilde{w}_{j \xi}^{R} & =-\tilde{g}_{u} \tilde{u}_{j-1}^{R}-\tilde{g}_{v} \tilde{v}_{j-1}^{R}+\ell \cdot o \cdot t .
\end{aligned}
$$

Here, $\tilde{g}_{u}=g_{u}\left(\tilde{u}_{0}^{R}, \tilde{v}_{0}^{R}\right)$, etc. By induction, the right side of (3.30) and (3.38) or (3.31) and (3.39) differs by $O\left(\left(1+|\xi|^{j-1}\right) e^{-\gamma|\xi|}\right)$. After integrating and subtracting, we have

$$
v_{j}^{S}-\tilde{v}_{j}^{R}=v_{j}^{S}(0)-\tilde{v}_{j}^{R}(0)+\int_{0}^{\xi} O\left(\left(1+|\xi|^{j-1}\right) e^{-\gamma|\xi|}\right) d \xi .
$$

Similar statements hold for $w$. This proves the lemma.

The matching of $\left(v_{j}^{S}, w_{j}^{S}\right)$ and $\left(\tilde{v}_{j}^{R}, \tilde{w}_{j}^{R}\right)$ from the right of $x_{0}^{i}$ becomes

$$
\begin{aligned}
v_{j}^{R}\left(x_{0}^{i}+\right)+v_{0 x}^{R}\left(x_{0}^{i}+\right) x_{j}^{i} & =v_{j}^{S}(0)+\ell \cdot o \cdot t, \\
w_{j}^{R}\left(x_{0}^{i}+\right)+w_{0 x}^{R}\left(x_{0}^{i}+\right) x_{j}^{i} & =w_{j}^{S}(0)+\ell \cdot o \cdot t,
\end{aligned}
$$

where $\ell \cdot o \cdot t$ are computable constant terms. From Lemma 3.3, The polynomials $P_{j}^{v}$ and $P_{j}^{w}$ in (3.36), (3.37) cancel with corresponding terms in (3.32), (3.33) with exponentially small errors. Similar formulas hold for the matching of $\left(v_{j}^{S}, w_{j}^{S}\right)$ and $\left(\tilde{v}_{j}^{R}, \tilde{w}_{j}^{R}\right)$ from the left of $x_{0}^{i}$. Therefore, (3.40), (3.41) and (3.35) lead to

$$
\begin{aligned}
& \mathbf{n}^{i} \cdot\left(v_{j}^{R}\left(x_{0}^{i}+\right)+v_{0 x}^{R}\left(x_{0}^{i}\right) x_{j}^{i}\right)=\mathbf{n}^{i} \cdot v_{j}^{S}(0)+\ell \cdot o \cdot t=\ell \cdot o \cdot t, \\
& {\left[v_{j}^{R}\right]\left(x_{0}^{i}\right)=\ell \cdot o \cdot t,} \\
& {\left[w_{j}^{R}\right]\left(x_{0}^{i}\right)+\left[w_{0 x}^{R}\right]\left(x_{0}^{i}\right) x_{j}^{i}=\ell \cdot o \cdot t .}
\end{aligned}
$$

Here we have used the fact $\left[v_{0 x}^{R}\right]\left(x_{0}^{i}\right)=0$.

(3.42), (3.43) and (3.44) form a complete set of interface conditions for $v_{j}^{R}$ which satisfies system (3.25). We still need boundary conditions for $v_{j}^{R}$. From (3.40) and (3.41) at $x_{0}^{0}=0$ and the fact $x_{j}^{0}=0$ for all $j \geq 1$, we have

$$
A_{0} w_{j}^{R}(0+)+B_{0} v_{j}^{R}(0+)=A_{0} w_{j}^{S 0}(0)+B_{0} v_{j}^{S 0}(0)+\ell \cdot o \cdot t=\ell \cdot o \cdot t .
$$

Similarly,

$$
A_{0} w_{j}^{R}(1-)+B_{0} v_{j}^{R}(1-)=A_{0} w_{j}^{S, r+1}(0)+B_{0} v_{j}^{S, r+1}(0)+\ell \cdot o \cdot t=\ell \cdot o \cdot t .
$$

Please refer to (3.11) and (3.12). From Lemma 3.2, with interface conditions (3.42), (3.43) and (3.44) at $x_{0}^{i}, 1 \leq i \leq r$, and boundary conditions (3.45) and (3.46), equation (3.25) can be uniquely solved. Once we have $v_{j}^{R}$, from (3.40) and (3.41) we can find $\left(v_{j}^{S}(0), w_{j}^{S}(0)\right)$. From (3.32) and (3.33) we can find $\left(v_{j}^{S}, w_{j}^{S}\right)$. We then find $u_{j}^{R}$ from (3.24)

The existence of $u_{j}^{S i}, 1 \leq i \leq r$, such that $\left|u_{j}^{S i}\right| \leq C\left(1+|\xi|^{j}\right)$ is guaranteed by condition (3.42), hence (3.35) and Lemma 2.3 In boundary layers, $i=0, r+1$, since $q^{i}=h^{i}\left(\bar{v}^{i}\right)$ are constants, from (H1), and the first part of Lemma 2.1, $u_{\xi \xi}+$ 
$D f\left(q^{i}\right) u=0$ has an exponential dichotomy. Also bounded solutions are in $W^{s}$ if $i=0$ or $W^{u}$ if $i=r+1$. From Lemma 2.1 again, bounded solutions with $u_{\xi}(0)=0$ must satisfy $u=0$. The existence of $u_{j}^{S i}, i=0, r+1$, with $\left|u_{j}^{S i}\right| \leq C\left(1+|\xi|^{j}\right)$ is a consequence of Lemma 2.2

The matching of $u^{S}$ and $u^{R}$ is satisfied due to the following lemma.

Lemma 3.4. If $\left|u_{j}^{S}-\tilde{u}_{j}^{R}\right| \leq C\left(1+|\xi|^{j}\right)$, then $\left|u_{j}^{S}-\tilde{u}_{j}^{R}\right| \leq C\left(\left(1+|\xi|^{j}\right) e^{-\gamma|\xi|}\right)$.

The proof of Lemma 3.4 uses Lemma 2.3 and can be found in [30].

\section{Critical eigenvalue and eigenfunctions}

The purpose of this section is to present a procedure to compute formal expansions of the critical eigenvalue and corresponding eigenfunctions $(\lambda(\epsilon), U(\epsilon), V(\epsilon))$. We need to solve (1.10) and (1.11) formally .

Analogous to the expansions of the layered solution, the critical eigenvalue and eigenfunctions are determined by three factors: a system of differential equations; boundary conditions in boundary layers; and the matching conditions. As in $\S 3$, the arguments of $f_{u}, f_{v}, g_{u}, g_{v}$ are $\left(u_{0}^{R}, v_{0}^{R}\right)$ in regular layers, and are $\left(u_{0}^{S}, v_{0}^{S}\right)$ in singular layers.

1. In regular layers,

$$
\begin{aligned}
& \lambda(\epsilon) U(\epsilon)=\epsilon^{2} U(\epsilon)_{x x}+f_{u} U(\epsilon)+f_{v} V(\epsilon), \\
& \lambda(\epsilon) V(\epsilon)=V(\epsilon)_{x x}+g_{u} U(\epsilon)+g_{v} V(\epsilon) .
\end{aligned}
$$

In singular layers, using the stretched variable $\xi$ as in (3.7), we have

$$
\begin{aligned}
\lambda(\epsilon) U(\epsilon) & =U(\epsilon)_{\xi \xi}+f_{u} U(\epsilon)+f_{v} V(\epsilon), \\
\epsilon^{2} \lambda(\epsilon) V(\epsilon) & =V(\epsilon)_{\xi \xi}+\epsilon^{2}\left(g_{u} U(\epsilon)+g_{v} V(\epsilon)\right) .
\end{aligned}
$$

Let $W=V_{x}$. Convert the $V$ equation into a first order system:

$$
\begin{aligned}
V_{\xi}(\epsilon) & =\epsilon W(\epsilon), \\
W_{\xi}(\epsilon) & =-\epsilon g_{u} U(\epsilon)-\epsilon g_{v} V(\epsilon)+\epsilon \lambda(\epsilon) V(\epsilon) .
\end{aligned}
$$

Denote the expansions in both regular and singular layers by

$$
\begin{aligned}
& U(\epsilon)=\sum_{j=0}^{\infty} \epsilon^{j} U_{j}, \\
& V(\epsilon)=\sum_{j=0}^{\infty} \epsilon^{j} V_{j} .
\end{aligned}
$$

We shall show later that $V_{0}^{S}(\xi)=0$. Thus, there is no $\epsilon^{-1}$ term in the expansion of $W^{S}=V_{\xi}^{S} / \epsilon$.

$$
W^{S}(\xi, \epsilon)=\sum_{j=0}^{\infty} \epsilon^{j} W_{j}^{S}(\xi) .
$$


2. The boundary conditions in the boundary layers are

$$
\begin{aligned}
& U_{x}(x, \epsilon)=0, \quad \text { for } x=0,1, \\
& A_{j} W(j, \epsilon)+B_{j} V(j, \epsilon)=0, \quad j=0,1,
\end{aligned}
$$

where $W=V_{x}$. Expanding in the powers of $\epsilon$, we find:

$$
\begin{aligned}
& U_{j \xi}^{S i}(0)=0, \quad i=0, r+1, \\
& A_{0} W_{j}^{S 0}(0)+B_{0} V_{j}^{S 0}(0)=0, \\
& A_{1} W_{j}^{S, r+1}(0)+B_{1} V_{j}^{S, r+1}(0)=0,
\end{aligned}
$$

for all $j \geq 0$. Using $W^{S}(\xi, \epsilon)=V_{\xi}^{S}(\xi, \epsilon) / \epsilon$, we have $W_{j}^{S}=V_{j+1, \xi}^{S}$ in the above.

3. Exponential matching principles.

Let $U^{R}$ be the outer solution in one of the regular layers adjacent to $x_{0}^{i}$. The inner expansion of $U^{R}$ is denoted by $\tilde{U}^{R}$.

$$
\begin{aligned}
& \sum_{0}^{\infty} \epsilon^{j} \tilde{U}_{j}^{R}(\xi)=U^{R}\left(\sum_{0}^{\infty} \epsilon^{j} x_{j}^{i}+\epsilon \xi, \epsilon\right), \\
& \sum_{0}^{\infty} \epsilon^{j} \tilde{V}_{j}^{R}(\xi)=V^{R}\left(\sum_{0}^{\infty} \epsilon^{j} x_{j}^{i}+\epsilon \xi, \epsilon\right) .
\end{aligned}
$$

The exponential matching principle.

$$
\begin{aligned}
\left|\tilde{U}_{j}^{R}(\xi)-U_{j}^{S}(\xi)\right|+\left|\tilde{U}_{j \xi}^{R}(\xi)-U_{j \xi}^{S}(\xi)\right| \leq C\left(1+|\xi|^{j}\right) e^{-\gamma|\xi|} \\
\left|\tilde{V}_{j}^{R}(\xi)-V_{j}^{S}(\xi)\right|+\left|\tilde{V}_{j \xi}^{R}(\xi)-V_{j \xi}^{S}(\xi)\right| \leq C\left(1+|\xi|^{j}\right) e^{-\gamma|\xi|} .
\end{aligned}
$$

Let $\sum \epsilon^{j} \tilde{W}_{j}^{R}(\xi)$ denote the inner expansion of $W^{R}$. (4.8) is equivalent to

$$
\left|\tilde{V}_{j}^{R}(\xi)-V_{j}^{S}(\xi)\right|+\left|\tilde{W}_{j}^{R}(\xi)-W_{j}^{S}(\xi)\right| \leq C\left(1+|\xi|^{j}\right) e^{-\gamma|\xi|} .
$$

(1) The $\epsilon^{0}$ th order expansion. Since $\lambda(\epsilon)$ is critical, $\lambda_{0}=0$.

In regular layers, from (4.1), (4.2),

$$
\begin{aligned}
& f_{u} U_{0}+f_{v} V_{0}=0, \\
& V_{0 x x}+g_{u} U_{0}+g_{v} V_{0}=0 .
\end{aligned}
$$

Therefore,

$$
\begin{aligned}
& U_{0}=-f_{u}^{-1} f_{v} V_{0}, \\
& V_{0 x x}-\left(g_{u} f_{u}^{-1} f_{v}-g_{v}\right) V_{0}=0 .
\end{aligned}
$$

In singular layers, from (4.4), 4.5)

$$
\begin{aligned}
& U_{0 \xi \xi}+f_{u} U_{0}+f_{v} V_{0}=0, \\
& V_{0 \xi}=0, \\
& W_{0 \xi}=0 .
\end{aligned}
$$

The last two equations imply that $V_{0}^{S}$ and $W_{0}^{S}$ are constants in singular layers. Form the matching principle,

$$
\left[V_{0}^{R}\right]\left(x_{0}^{i}\right)=\left[W_{0}^{R}\right]\left(x_{0}^{i}\right)=0, \quad 1 \leq i \leq r
$$


In the boundary layers, the constant solution $\left(V_{0}^{S}, W_{0}^{S}\right)=\left(V_{0}^{S}(0), W_{0}^{S}(0)\right)$ matches with $V_{0}^{R}(x), W_{0}^{R}(x), x=0,1$, respectively. Therefore, from (4.6), the boundary conditions for $V_{0}^{R}$ are

$$
A_{j} V_{x}(j)+B_{j} V(j)=0, \quad j=0,1 .
$$

We need the following hypothesis,

(H7) If $V \in C^{1}([0,1]) \cap C^{2}\left(\left(x_{0}^{i}, x_{0}^{i+1}\right), 0 \leq i \leq r\right.$, then $V=0$ is the only solution for the following boundary value problem:

$$
\begin{aligned}
& V_{x x}-\left(g_{u} f_{u}^{-1} f_{v}-g_{v}\right) V=0, \\
& A_{j} V_{x}(j)+B_{j} V(j)=0, \quad j=0,1 .
\end{aligned}
$$

We comment that if (H7) is not satisfied, then the regular eigenvalues, which solve the reduced eigenvalue problem (7.1), will have $\lambda=0$ as a root. In this case, asymptotic expansions of critical eigenvalues are quite different and will not be touched in this paper. In $\S 7$, a stronger assumption (H9), which implies (H7), will be imposed to ensure that the regular eigenvalues are in the region $\operatorname{Re} \lambda \leq-\gamma<0$.

From (H7), we can prove the following lemma:

Lemma 4.1. Assume that $V$ satisfies

$$
\begin{aligned}
& V_{x x}-\left(g_{u} f_{u}^{-1} f_{v}-g_{v}\right) V=E_{1}, \\
& {[V]\left(x_{0}^{i}\right)=E_{2},} \\
& {\left[V_{x}\right]\left(x_{0}^{i}\right)=E_{3},} \\
& A_{j} V_{x}(j)+B_{j} V(j)=E_{4 j}, \quad j=0,1 .
\end{aligned}
$$

Here $E_{2}, E_{3}, E_{4 j} \in \mathbb{R}^{n}, E_{1} \in C\left(\left(x_{0}^{i}, x^{i+1}\right)\right), 0 \leq i \leq r$, and has one-sided limits at the boundary points. Then there exists a unique piecewise $C^{2}$ solution $V \in$ $C^{1}([0,1]) \cap C^{2}\left(\left(x_{0}^{i}, x_{0}^{i+1}\right)\right), 0 \leq i \leq r$.

Proof. The general solution for the first equation in $r+1$ intervals has $2 n(r+1)$ parameters which must be determined by a linear system of algebraic equation derived from the other 4 equations. It is easy to verify that the Fredholm index of this algebraic system is zero, therefore, it suffices to prove that the linear homogeneous system has only the zero solution. The latter follows from (H7).

It follows from (H7) that $V_{0}^{R}=0$ on $[0,1]$, and hence $V_{0}^{S}=0$ in all the singular layers. With $V_{0}^{S}=0$, (4.10) has a bounded solution $U_{0}^{S}=c_{0}^{i} \dot{q}^{i}$ in the $i$ th singular layer. When $i=0$ or $r+1, U_{0}^{S i}=\dot{q}^{i}=0$, which satisfies the Neumann boundary conditions. We shall assume $c_{0}^{0}=c_{0}^{r+1}=1$. It is clear that the matching of inner and outer solutions are also satisfied.

To summarize,

$$
\begin{aligned}
\lambda_{0}=0, & \text { critical eigenvalue, } \\
V_{0}^{R}=0, U_{0}^{R}=0, & \text { in regular layers, } \\
V_{0}^{S}=0, U_{0}^{S}=c_{0}^{i} \dot{q}^{i}, & \text { in the } i \text { th singular layers. }
\end{aligned}
$$

The constants $c_{0}^{0}=c_{0}^{r+1}=1$, but $\left\{c_{0}^{i}\right\}_{1}^{r}$ remain to be determined. 
(2) The $\epsilon^{1}$ th order expansion. In the regular layers, since $\lambda_{0} U_{1}^{R}+\lambda_{1} U_{0}^{R}=0=$ $\lambda_{0} V_{1}^{R}+\lambda_{1} V_{0}^{R}$. We have, from (4.1), (4.2),

$$
\begin{aligned}
& f_{u} U_{1}^{R}+f_{v} V_{1}^{R}=0, \\
& V_{1 x x}^{R}+g_{u} U_{1}^{R}+g_{v} V_{1}^{R}=0 .
\end{aligned}
$$

Therefore,

$$
\begin{aligned}
& U_{1}^{R}=-f_{u}^{-1} f_{v} V_{1}^{R}, \\
& V_{1 x x}^{R}-\left(g_{u} f_{u}^{-1} f_{v}-g_{v}\right) V_{1}^{R}=0 .
\end{aligned}
$$

In the $i$ th singular layer, the equations for $\left(U_{1}^{S}, V_{1}^{S}, W_{1}^{S}\right)$ become

$$
\begin{aligned}
& \lambda_{1} c_{0}^{i} \dot{q}^{i}=U_{1 \xi \xi}^{S}+f_{u} U_{1}^{S}+f_{v} V_{1}^{S}+c_{0}^{i}\left(f_{u u} \dot{q}^{i} u_{1}^{S}+f_{u v} \dot{q}^{i} v_{1}^{S}\right), \\
& V_{1 \xi}^{S}=W_{0}^{S}=0, \\
& W_{1 \xi}^{S}=-g_{u} U_{0}^{S}-g_{v} V_{0}^{S}=-g_{u} c_{0}^{i} \dot{q}^{i} .
\end{aligned}
$$

From (4.14), (4.15),

$$
\begin{aligned}
& V_{1}^{S}=\text { constant }=V_{1}^{R}\left(x_{0}^{i}\right), \\
& W_{1}^{S}(\infty)-W_{1}^{S}(-\infty)=-c_{0}^{i} \int_{-\infty}^{\infty} g_{u} \dot{q}^{i}(\xi) d \xi, \quad 1 \leq i \leq r .
\end{aligned}
$$

Let $\mathcal{M}^{i}=g\left(q^{i}(-\infty), \bar{v}^{i}\right)-g\left(q^{i}(\infty), \bar{v}^{i}\right)$. By the matching principle,

$$
\begin{aligned}
& {\left[V_{1}^{R}\right]\left(x_{0}^{i}\right)=0,} \\
& {\left[V_{1 x}^{R}\right]\left(x_{0}^{i}\right)=c_{0}^{i} \mathcal{M}^{i} .}
\end{aligned}
$$

In the boundary layers, $\dot{q}^{i}=0, i=0, r+1$. Thus $\left(V_{1}^{S}, W_{1}^{S}\right)$ are constants solutions in the boundary layers. By the matching conditions and (4.6), the boundary conditions for $V_{1}^{R}$ are again (4.11).

From Lemma 4.1, there exist solution $V_{c}^{i}, 1 \leq i \leq r$, of (4.12) that satisfies

$$
\begin{aligned}
& {[V]\left(x_{0}^{\nu}\right)=0, \quad \text { for all } \nu,} \\
& {\left[V_{x}\right]\left(x_{0}^{\ell}\right)=0, \quad \text { for all } \ell \neq i,} \\
& {\left[V_{x}\right]\left(x_{0}^{i}\right)=\mathcal{M}^{i} .}
\end{aligned}
$$

By the superposition principle,

$$
V_{1}^{R}=\sum_{1}^{r} c_{0}^{i} V_{c}^{i}
$$

In order to find a solution $U_{1}^{S}=O(1+|\xi|)$ for (4.13), the nonhomogeneous terms must be in the range of a Fredholm operator, see Lemma 2.3. Therefore

$$
\lambda_{1} c_{0}^{i}\left\langle\psi^{i}, \dot{q}^{i}\right\rangle=\left\langle\psi^{i}, f_{v} V_{1}^{S}+c_{0}^{i}\left(f_{u u} \dot{q}^{i} u_{1}^{S}+f_{u v} \dot{q}^{i} v_{1}^{S}\right)\right\rangle .
$$

The above can be simplified using integration by parts as follows:

$$
\begin{aligned}
f_{u u} \dot{q}^{i} u_{1}^{S}+f_{u v} \dot{q}^{i} v_{1}^{S} & =\partial_{\xi}\left(f_{u} u_{1}^{S}+f_{v} v_{1}^{S}\right)-f_{u} u_{1 \xi}^{S}-f_{v} v_{1 \xi}^{S} \\
& =\partial_{\xi}\left(-u_{1 \xi \xi}^{S}\right)-f_{u} u_{1 \xi}^{S}-f_{v} v_{1 \xi}^{S} \\
& =-\left\{\left(u_{1 \xi}^{S}\right)_{\xi \xi}+f_{u}\left(u_{1 \xi}^{S}\right)\right\}-f_{v} v_{1 \xi}^{S} .
\end{aligned}
$$


Since the term in the \{\} is in the range of a Fredholm operator, we have

$$
\left\langle\psi^{i},\left\{\left(u_{1 \xi}^{S}\right)_{\xi \xi}+f_{u}\left(u_{1 \xi}^{S}\right)\right\}\right\rangle=0 .
$$

Therefore,

$$
\begin{gathered}
\left\langle\psi^{i},\left(f_{u u} \dot{q}^{i} u_{1}^{S}+f_{u v} \dot{q}^{i} v_{1}^{S}\right)\right\rangle=-\left\langle\psi^{i}, f_{v} v_{1 \xi}^{S}\right\rangle . \\
c_{0}^{i} \lambda_{1}\left\langle\psi^{i}, \dot{q}^{i}\right\rangle=\left\langle\psi^{i}, f_{v}\left(V_{1}^{S}-c_{0}^{i} v_{1 \xi}^{S}\right)\right\rangle .
\end{gathered}
$$

If we recall that $\left\langle\psi^{i}, \dot{q}^{i}\right\rangle=1, \mathbf{n}^{i}=\left\langle\psi^{i}, f_{v}\right\rangle$ and $V_{1}^{S}$ and $v_{1 \xi}^{S}$ are constants, we have $c_{0}^{i} \lambda_{1}=\mathbf{n}^{i} \cdot\left(V_{1}^{S}-c_{0}^{i} v_{1 \xi}^{S}\right)$. Using $v_{1 \xi}^{S}=w_{0}^{S}=w_{0}^{R}\left(x_{0}^{i}\right)=v_{0 x}^{R}\left(x_{0}^{i}\right), V_{1}^{S i}=V_{1}^{R}\left(x_{0}^{i}\right)$, we have

$$
c_{0}^{i} \lambda_{1}=\mathbf{n}^{i} \cdot\left(V_{1}^{R}\left(x_{0}^{i}\right)-c_{0}^{i} v_{0 x}^{R}\left(x_{0}^{i}\right)\right) .
$$

From (4.18), equation (4.20) becomes

$$
\lambda_{1} c_{0}^{i}=\mathbf{n}^{i} \cdot\left(\sum_{\ell=1}^{r} c_{0}^{\ell} V_{c}^{\ell}\left(x_{0}^{i}\right)-c_{0}^{i} w_{0}^{R}\left(x_{0}^{i}\right)\right), \quad i=1,2, \cdots, r .
$$

Define the coupling matrix $A=\left(a_{i \ell}\right)_{r \times r}$ by

$$
a_{i \ell}=\mathbf{n}^{i} \cdot\left(V_{c}^{\ell}\left(x_{0}^{i}\right)-\delta_{i \ell} v_{0 x}^{R}\left(x_{0}^{i}\right)\right) .
$$

We comment that $A$ is precisely the SLEP matrix in the NF model. We see that $\lambda_{1}$ is an eigenvalue while $\left(c_{0}^{1}, c_{0}^{2}, \cdots, c_{0}^{r}\right)^{\tau}$ is an eigenvector for $A$.

$$
\lambda_{1}\left(\begin{array}{c}
c_{0}^{1} \\
\vdots \\
c_{0}^{r}
\end{array}\right)=A\left(\begin{array}{c}
c_{0}^{1} \\
\vdots \\
c_{0}^{r}
\end{array}\right) .
$$

To construct higher order expansions, we may use any of the $r$ eigenvalues and the corresponding eigenvectors of $A$. With such $\lambda_{1}$ and $\left(c_{0}^{1}, c_{0}^{2}, \cdots, c_{0}^{r}\right)$, (4.13) has a solution $|U| \leq C(1+|\xi|)$ which can be written as

$$
U_{1}^{S}=Z_{1}^{S}+c_{1}^{i} \dot{q}^{i} .
$$

Here $\left\langle\dot{q}^{i}, Z_{1}^{i}\right\rangle=0$, and the parameters $\left\{c_{1}^{i}\right\}_{1}^{r}$ remain to be determined.

Finally, in the boundary layers, (4.13) becomes

$$
U_{1 \xi \xi}+f_{u} U_{1}+f_{v} V_{1}=0
$$

With $V_{1}^{S}$ already obtained, there exists a unique solution $U_{1}^{S}=O(1+|\xi|)$ in the boundary layers. See Lemma 2.2.

(3) The $\epsilon^{j}$ th order expansions, $j \geq 2$. Assume that we have computed

$$
\lambda_{0}, \lambda_{1}, \cdots, \lambda_{j-1} \text {. }
$$

We have obtained in regular layers

$$
\begin{aligned}
& U_{0}, U_{1}, \cdots, U_{j-1}, \\
& V_{0}, V_{1}, \cdots, V_{j-1}, \\
& W_{0}, W_{1}, \cdots, W_{j-1} .
\end{aligned}
$$

In singular layers, we have computed all the above except for $U_{j-1}^{S}$ which, in the $i$ th internal layer, has the form

$$
U_{j-1}^{S i}=c_{j-1}^{i} \dot{q}^{i}+Z_{j-1}^{i}, \quad\left\langle\dot{q}^{i}, Z_{j-1}^{i}\right\rangle=0 .
$$


Assume that $Z_{j-1}^{i}$ has been determined but $c_{j-1}^{i}$ is still a free parameter. In the $\epsilon^{j}$ th expansion, we will determine $\lambda_{j},\left\{c_{j-1}^{i}\right\}_{1}^{r}, V_{j}, W_{j}$ and $U_{j}^{R}$. We will determine $U_{j}^{S}$ up to $c_{j}^{i} \dot{q}^{i}$.

Definition. An eigenfunction $(U(\epsilon), V(\epsilon))$ is called a normalized eigenfunction if the corresponding parameters $\left\{c_{j}^{i}\right\}_{1}^{r}$ satisfy

$$
\begin{aligned}
& \sum_{i=1}^{r}\left(c_{0}^{i}\right)^{2}=1, \\
& \left(c_{\ell}^{1}, c_{\ell}^{2}, \cdots, c_{\ell}^{r}\right) \perp\left(c_{0}^{1}, c_{0}^{2}, \cdots, c_{0}^{r}\right), \quad \ell \geq 1 .
\end{aligned}
$$

It is not hard to verify that if $(U(\epsilon), V(\epsilon))$ is a normalized eigenfunction, and if $\alpha(\epsilon)=\sum \epsilon^{j} \alpha_{j}$ is a scalar series, then $(\alpha(\epsilon) U(\epsilon), \alpha(\epsilon) V(\epsilon))$ is the general form of all the eigenfunctions. In the sequel, we will assume that the eigenfunctions are normalized.

In the regular layer, since $\lambda_{0}=0$ and $U_{0}^{R}=V_{0}^{R}=0$,

$$
\begin{aligned}
& \lambda_{j} U_{0}^{R}+\cdots+\lambda_{0} U_{j}^{R}=\ell \cdot o \cdot t, \\
& \lambda_{j} V_{0}^{R}+\cdots+\lambda_{0} V_{j}^{R}=\ell \cdot o \cdot t .
\end{aligned}
$$

Therefore,

$$
\begin{aligned}
& f_{u} U_{j}^{R}+f_{v} V_{j}^{R}=\ell \cdot o \cdot t, \\
& V_{j x x}^{R}+g_{u} U_{j}^{R}+g_{v} V_{j}^{R}=\ell \cdot o \cdot t, \\
& U_{j}^{R}=-f_{u}^{-1} f_{v} V_{j}^{R}+\ell \cdot o \cdot t, \\
& V_{j x x}^{R}-\left(g_{u} f_{u}^{-1} f_{v}-g_{v}\right) V_{j}^{R}=\ell \cdot o \cdot t .
\end{aligned}
$$

From (H7), $V_{j}^{R}$ can be uniquely solved for if the boundary conditions at $x=0,1$ and the jumps across $\left\{x_{0}^{i}\right\}_{i=1}^{r}$ can be found. The jumps can be found by matching the internal and regular layers.

In the $i$ th internal layer, since $U_{j-1}^{S}=c_{j-1}^{i} \dot{q}^{i}+Z_{j-1}^{i}$ and $V_{0}^{S}=0$,

$$
\begin{gathered}
\lambda_{1} U_{j-1}^{S}+\cdots+\lambda_{j} U_{0}^{S}=c_{j-1}^{i} \lambda_{1} \dot{q}^{i}+c_{0}^{i} \lambda_{j} \dot{q}^{i}+\ell \cdot o \cdot t, \\
\lambda_{0} V_{j}^{S}+\cdots \lambda_{j} V_{0}^{S}=\ell \cdot o \cdot t . \\
c_{j-1}^{i} \lambda_{1} \dot{q}^{i}+c_{0}^{i} \lambda_{j} \dot{q}^{i}=U_{j \xi \xi}^{S}+f_{u} U_{j}^{S}+f_{v} V_{j}^{S} \\
\quad+f_{u u} c_{j-1}^{i} \dot{q}^{i} u_{1}+f_{u v} c_{j-1}^{i} \dot{q}^{i} v_{1}+\ell \cdot o \cdot t . \\
V_{j \xi}^{S}=W_{j-1}^{S}, \\
W_{j \xi}^{S}=-g_{u} U_{j-1}^{S}-g_{v} V_{j-1}^{S}+\ell \cdot o \cdot t, \\
=-c_{j-1}^{i} g_{u} \dot{q}^{i}+\ell \cdot o \cdot t, \\
V_{j}^{S}(\xi)=V_{j}^{S}(0)+\int_{0}^{\xi} \ell \cdot o \cdot t, \\
W_{j}^{S}(\xi)=W_{j}^{S}(0)+\int_{0}^{\xi}\left(-c_{j-1}^{i} g_{u} \dot{q}^{i}\right)+\ell \cdot o \cdot t,
\end{gathered}
$$

Notice that $\left(V_{j}^{S}, W_{j}^{S}\right)$ behaves like a polynomial of degree $j$ as $|\xi| \rightarrow \infty$. The matching of higher powers of $\xi$ can be proved by induction, see Lemma 3.3. We only have to match the constant terms. Integrating from $\xi=-\infty$ to $\infty$, and 
applying the matching principles, similar to (3.43) and (3.44), we conclude that for $1 \leq i \leq r$

$$
\begin{aligned}
{\left[V_{j}^{R}\right]\left(x_{0}^{i}\right) } & =\ell \cdot o \cdot t, \\
{\left[W_{j}^{R}\right]\left(x_{0}^{i}\right) } & =c_{j-1}^{i} \mathcal{M}^{i}+\ell \cdot o \cdot t .
\end{aligned}
$$

In the boundary layers, since $\dot{q}^{i}=0, i=0, r+1$, from (4.26), (4.27), and the matching of outer and inner layers, we have

$$
\begin{aligned}
V_{j}^{R}(0+) & =V_{j}^{S 0}(0)+\ell \cdot o \cdot t, \\
W_{j}^{R}(0+) & =W_{j}^{S 0}(0)+\ell \cdot o \cdot t .
\end{aligned}
$$

Therefore the boundary condition at $x=0$ can be obtained,

$$
A_{0} W_{j}^{R}(0)+B_{0} V_{j}^{R}(0)=\ell \cdot o \cdot t .
$$

Similarly, at $x=1$,

$$
A_{1} W_{j}^{R}(1)+B_{1} V_{j}^{R}(1)=\ell \cdot o \cdot t .
$$

With all the boundary and jump conditions, based on (H7), we can solve for $V_{j}^{R}$ from (4.22). Using the superposition principle and the basis functions $\left\{V_{c}^{i}\right\}$, we can express the solution as a function of $\left\{c_{j-1}^{i}\right\}_{1}^{r}$.

$$
V_{j}^{R}=\sum_{i=1}^{r} c_{j-1}^{i} V_{c}^{i}+\ell \cdot o \cdot t
$$

In order to have a solution $\left|U_{j}^{S i}\right| \leq C\left(1+|\xi|^{j}\right), 1 \leq i \leq r$, for (4.23), the nonhomogeneous terms must be in the range of a Fredholm operator, see Lemma 2.3. This leads to

$$
c_{0}^{i} \lambda_{j}+c_{j-1}^{i} \lambda_{1}=\left\langle\psi^{i}, f_{v} V_{j}^{S}+c_{j-1}^{i}\left(f_{u u} \dot{q}^{i} u_{1}+f_{u v} \dot{q}^{i} v_{1}\right)\right\rangle+\ell \cdot o \cdot t .
$$

Using integration by parts similar to (4.19),

$$
c_{0}^{i} \lambda_{j}+c_{j-1}^{i} \lambda_{1}=\left\langle\psi^{i}, f_{v}\left(V_{j}^{S}-c_{j-1}^{i} w_{0}^{R}\left(x_{0}^{i}\right)\right)\right\rangle+\ell \cdot o \cdot t .
$$

From (4.26), we then have

$$
c_{0}^{i} \lambda_{j}+c_{j-1}^{i} \lambda_{1}=\left\langle\psi^{i}, f_{v}\left(V_{j}^{S}(0)-c_{j-1}^{i} w_{0}^{R}\left(x_{0}^{i}\right)\right)\right\rangle+\ell \cdot o \cdot t .
$$

By the matching principle and (4.26),

$$
V_{j}^{S}(0)=V_{j}^{R}\left(x_{0}^{i}+\right)+\ell \cdot o \cdot t .
$$

Thus

$$
c_{0}^{i} \lambda_{j}+c_{j-1}^{i} \lambda_{1}=\left\langle\psi^{i}, f_{v}\left(V_{j}^{R}\left(x_{0}^{i}+\right)-c_{j-1}^{i} w_{0}^{R}\left(x_{0}^{i}\right)\right)\right\rangle+\ell \cdot o \cdot t .
$$

Using (4.28) we have

$$
c_{0}^{i} \lambda_{j}+c_{j-1}^{i} \lambda_{1}=\mathbf{n}^{i} \cdot\left(\sum_{\ell=1}^{r} c_{j-1}^{\ell} V_{c}^{\ell}\left(x_{0}^{i}\right)-c_{j-1}^{i} w_{0}^{R}\left(x_{0}^{i}\right)\right)+\ell \cdot o \cdot t .
$$

In the matrix form

$$
\left(\lambda_{1} I-A\right)\left(\begin{array}{c}
c_{j-1}^{1} \\
\vdots \\
c_{j-1}^{r}
\end{array}\right)=\lambda_{j}\left(\begin{array}{c}
c_{0}^{1} \\
\vdots \\
c_{0}^{r}
\end{array}\right)+\ell \cdot o \cdot t .
$$

We need the following hypothesis. 
(H8) $\lambda_{1}$ is a pole of order one for the matrix $\lambda I-A$. (The Jordan blocks of $\lambda I-A$ corresponding to $\lambda_{1}$ are of order 1 .)

Remark. If (H8) is not satisfied, then $\lambda(\epsilon)$ may not be expanded as integer powers of $\epsilon$. A discussion of asymptotic expansions for eigenvalues of an $\epsilon$ dependent matrix can be found in 20 .

Condition (H8) is always satisfied if all the eigenvalues of the coupling matrix are distinct, which is certainly true if mono-internal layer solutions are considered.

Based on (H8), $\left(c_{0}^{1}, \cdots, c_{0}^{r}\right)$ is not in the range of $\lambda_{1} I-A$. (4.32) uniquely determines $\lambda_{j}$ and $\left\{c_{j-1}^{i}\right\}_{1}^{r}$, due to the normalization

$$
\left(c_{j-1}^{1}, \cdots, c_{j-1}^{r}\right) \perp\left(c_{0}^{1}, \cdots, c_{0}^{r}\right) .
$$

It is clear with such $\lambda_{j}$ and $\left\{c_{j-1}^{i}\right\}_{1}^{r}$, we can uniquely find $Z_{j}^{i}=O\left(1+|\xi|^{j}\right),\left\langle\dot{q}^{i}, Z_{j}^{i}\right\rangle=$ 0 , such that the solution for (4.23) has the form,

$$
U_{j}^{S}=Z_{j}^{i}+c_{j}^{i} \dot{q}^{i}
$$

in the $i$ th internal layers.

$V_{j}^{R}$ then comes from (4.28). $V_{j}^{S}$ comes from (4.26). After obtaining $V_{j}^{S}$, in the boundary layers, since $\dot{q}^{i}=0, i=0, r+1$, the equation for $U_{j}^{S}$ becomes

$$
U_{j \xi \xi}^{S}+f_{u} U_{j}^{S}=\ell \cdot o \cdot t
$$

Since the right hand side is of $O\left(1+|\xi|^{j}\right)$, the above equation with Neumann boundary conditions can be uniquely solved for a solution $U=O\left(1+|\xi|^{j}\right)$ in $\mathbb{R}^{+}$ or $\mathbb{R}^{-}$respectively for $i=0$, or $i=r+1$. See Lemma 2.2

We summarize our result in the following theorem:

Theorem 4.2. Assume that (H1)-(H7) are satisfied, than the asymptotic expansion of critical eigenvalues $\lambda$ and eigenfunctions $(U, V)$ can be obtained up to $\epsilon^{1} . \lambda_{1}$ is an eigenvalue for the coupling matrix $A$. The associated eigenvector $\left\{c_{0}^{i}\right\}_{1}^{n}$ provides information about the eigenfunction $(U, V)$, which satisfies $U_{0}^{R}=0, V_{0}^{R}=0$ and $V_{1}^{R}=\sum c_{0}^{i} V_{c}^{i}$ in regular layers, and $U_{0}^{S i}(\xi)=c_{0}^{i} \dot{q}^{i}(\xi)$ and $V_{0}^{S}=0$ in the singular layer at $x_{0}^{i}$.

Assume that (H8) is also satisfied. Then the higher order expansion of critical eigenvalues and the corresponding eigenfunctions can be obtained by a recursive procedure to any power of $\epsilon$.

The formal series expansions of eigenvalues and eigenfunctions satisfy (4.1), (4.2) in regular layers, 4.3), 4.4) in singular layers, boundary conditions (4.6) and matching conditions (4.7), (4.8).

\section{Mono-LAYER SOlutions AND A GEOMETRIC METHOD}

We first introduce a geometric method to determine mono-layer solutions. We show that the geometric method also determines $\lambda_{1}$, hence, the stability of the mono-layer solution. At the end of this section, we comment on the relation of our approach with the geometric singular perturbation theory.

Let $\Phi_{-}$and $\Phi_{+}$be the solution maps of (3.6) where $h=h^{0}$ and $h=h^{1}$ respectively for all $x$. Since $\Phi_{-}$and $\Phi_{+}$are transverse to $\Gamma^{0}$ and $\Gamma^{2}$, the following are 
$(n+1)$-dimensional manifolds,

$$
\begin{aligned}
& \mathcal{M}_{-}=\bigcup\left\{\Phi_{-}(x) \mathcal{S}_{0} \mid 0 \leq x \leq 1\right\}, \\
& \mathcal{M}_{+}=\bigcup\left\{\Phi_{+}(x-1) \mathcal{S}_{1} \mid 0 \leq x \leq 1\right\} .
\end{aligned}
$$

Lemma 5.1. If (H4)-(H6) are satisfied, then $\mathcal{M}_{-}$intersects with $\mathcal{M}_{+}$transversely. The intersection $\mathcal{C}$ is a smooth one-dimensional curve that satisfies $\mathcal{C} \pitchfork \Gamma^{1}$.

Conversely, if $\mathcal{M}_{-} \pitchfork \mathcal{M}_{+}$and the intersection $\mathcal{C}$ satisfies $\mathcal{C} \pitchfork \Gamma^{1}$, then (H4)-(H6) are satisfied.

Proof. Denote $\wp=\left(v^{\dagger}, w^{\dagger}, x^{\dagger}\right)$ the intersection of $\mathcal{C}$ and $\Gamma^{1}$. Since $\Gamma^{1}$ is of codimension one, if $T_{\wp} \mathcal{M}_{-} \cap T_{\wp} \mathcal{M}_{+}$is two dimensional, then there exists a nonzero vector

$$
\mathbf{a} \in T_{\wp} \mathcal{M}_{-} \cap T_{\wp} \mathcal{M}_{+} \cap T_{\wp} \Gamma^{1} .
$$

Therefore, $\mathbf{a} \in T_{\wp} \mathcal{S}_{-}^{1}$. From (H5), the flow at $\wp$ is transverse to $\mathcal{S}_{-}^{1}$. The derivative of the Poincaré mapping $\mathcal{P}^{1}$ will send a to a vector in the tangent spaces of both $\mathcal{S}_{-}^{2}$ and $\mathcal{S}_{1}$. From $(\mathrm{H} 6)$, it must be a zero vector. The contradiction shows that $\mathcal{M}_{-} \pitchfork \mathcal{M}_{+}$.

We now show that $\mathcal{C} \pitchfork \Gamma^{1}$. Assume a vector $\mathbf{a} \in T_{\wp} \mathcal{C} \subset T_{\wp} \Gamma^{1}$, then as the above, $\mathbf{a} \in \mathcal{S}_{-}^{1} \cap \mathcal{M}_{+}$. Thus, as before, $\mathbf{a}=0$. This shows that $\mathcal{C} \pitchfork \Gamma^{1}$.

The converse of the lemma can be proved by a similarly elementary argument and will not be given here.

The curve $\mathcal{C} \stackrel{\text { def }}{=} \mathcal{M}_{-} \cap \mathcal{M}_{+}$is called a slow switching curve since the slow flow has to switch from $u=h^{0}(v)$ to $h^{1}(v)$ at some $\wp \in \mathcal{C}$ in order to satisfy boundary conditions at $x=0,1$. $\mathcal{C}$ is not a solution curve of (3.6) if the slow equation has a jump caused by $h^{0} \neq h^{1}$.

We have obtained the following theorem.

Theorem 5.2. Assume that (H1)-(H3) are satisfied and $\mathcal{C} \pitchfork \Gamma^{1}$ at some nonempty point $\wp=\left(v^{\dagger}, w^{\dagger}, x^{\dagger}\right)$. Then there exists a singular mono-internal layer solution with the internal layer at $x=x^{\dagger}$ and $\left(v\left(x^{\dagger}\right), w\left(x^{\dagger}\right)\right)=\left(v^{\dagger}, w^{\dagger}\right)$. Moreover, the asymptotic expansions of the mono-internal layer solution to any powers of $\epsilon$ can be calculated recursively as in $\S 3$.

Let $(\Delta v, \Delta w, \Delta x)$ be a nonzero tangent vector of $\mathcal{C}$ at $\wp$. We can show that $\Delta x \neq 0$. For otherwise using $(\Delta v, \Delta w) \neq 0$ as an initial condition at $x=x_{0}$, the linear system

$$
\begin{aligned}
V_{x} & =W, \\
W_{x} & =\left(g_{u} f_{u}^{-1} f_{v}-g_{v}\right) V,
\end{aligned}
$$

where $h=h^{0}$ if $x<0, h=h^{1}$ if $x>x_{0}$, has a nontrivial solution that is $C^{1}$ on $[0,1]$. This is a contradiction to (H7).

After rescaling, assume that $\mathbf{t}=(\Delta v, \Delta w,-1)$ is a tangent vector of $\mathcal{C}$ at $\wp$. We have the following simple result.

Theorem 5.3. $\lambda_{1}=\mathbf{n}^{1} \cdot \Delta v$ where $\mathbf{n}^{1}$ is the normal of the surface $\Sigma^{1}$ as in $\S 3$ and $(\Delta v, \Delta w,-1)$ is a tangent vector of $\mathcal{M}_{-} \cap \mathcal{M}_{+}$at $\left(v_{0}^{R}\left(x_{0}\right), w_{0}^{R}\left(x_{0}\right), x_{0}\right)$. Let $\mathbf{N}=\left(\mathbf{n}^{1}, 0,0\right)$ be a normal of $\Gamma^{1}$. The result can also be expressed as

$$
\lambda_{1}=\mathbf{t} \cdot \mathbf{N} \text {. }
$$


Proof. Since there is only one internal layer, we drop the super-index $i=1$ for the layer. Let $\left(V_{c}, W_{c}\right)$ be a solution of the system (4.12) with

$$
\begin{aligned}
& {[V]\left(x_{0}\right)=0,} \\
& {[W]\left(x_{0}\right)=g(q(-\infty), \bar{v})-g(q(\infty), \bar{v}) .}
\end{aligned}
$$

Then

$$
\begin{aligned}
& \left(V_{c}\left(x_{0}\right), W_{c}\left(x_{0}-\right), 0\right) \in T_{\wp} \mathcal{M}_{-}, \\
& \left(V_{c}\left(x_{0}\right), W_{c}\left(x_{0}+\right), 0\right) \in T_{\wp} \mathcal{M}_{+} .
\end{aligned}
$$

The following two vectors are equal,

$$
\begin{aligned}
&\left(V_{c}\left(x_{0}\right)-w_{0}^{R}\left(x_{0}\right), W_{c}\left(x_{0}-\right)+g(q(-\infty), \bar{v}),-1\right) \\
& \quad=\left(V_{c}\left(x_{0}\right)-w_{0}^{R}\left(x_{0}\right), W_{c}\left(x_{0}+\right)+g(q(\infty), \bar{v}),-1\right) .
\end{aligned}
$$

It is clear that

$$
\begin{aligned}
\left(-w_{0}^{R}\left(x_{0}\right), g(q(-\infty), \bar{v}),-1\right) & \in T_{\wp} \mathcal{M}_{-}, \\
\left(-w_{0}^{R}\left(x_{0}\right), g(q(\infty), \bar{v}),-1\right) & \in T_{\wp} \mathcal{M}_{+},
\end{aligned}
$$

since they are flows of $\Phi_{-}$and $\Phi_{+}$respectively. Therefore the common vector in (5.1) is in

$$
T_{\wp} \mathcal{C}=T_{\wp} \mathcal{M}_{-} \cap T_{\wp} \mathcal{M}_{+} .
$$

Thus, we must have $V_{c}\left(x_{0}\right)-w_{0}^{R}\left(x_{0}\right)=\Delta v$. The desired result now follows from Theorem 4.2 where

$$
A=\left(a_{11}\right)=\mathbf{n}^{1} \cdot\left(V_{c}\left(x_{0}\right)-w_{0}^{R}\left(x_{0}\right)\right)=\mathbf{n}^{1} \cdot \Delta v .
$$

A similar theorem can be stated for the existence of a singular heteroclinic solution which has an internal layer. Let $\left(p^{i}, 0\right)$ be a hyperbolic equilibrium for the reduced system $v^{\prime}=w, w^{\prime}=-g\left(h^{i}(v), v\right)$ with $i=0,1$. Assume that $\operatorname{dim} W^{u}\left(\left(p^{0}, 0\right)\right)-\operatorname{dim} W^{u}\left(\left(p^{1}, 0\right)\right)=1$ where the stable unstable manifolds of $\left(p^{i}, 0\right)$ are referred to the vector fields with $h=h^{i}$. Assume the nonempty transversal intersection of $W^{u}\left(\left(p^{0}, 0\right)\right)$ and $W^{s}\left(\left(p^{1}, 0\right)\right)$ on $\mathbb{R}^{2 n}$. Then $\mathcal{C} \stackrel{\text { def }}{=} W^{u}\left(p^{0}\right) \cap W^{s}\left(p^{1}\right)$ is a smooth one dimensional curve. Define $\Gamma^{1}$ to be the set of points $(v, w) \in \mathbb{R}^{2 n}$ where $u^{\prime \prime}+f(u, v)=0$ has a heteroclinic solution connecting $u=h^{0}(v)$ to $h^{1}(v)$.

We can show if $\mathcal{C} \pitchfork \Gamma^{1}$ at a nonempty point, then there exists a singular internal layer solution connecting $(u, v)=\left(h^{0}\left(p^{0}\right), p^{0}\right)$ to $(u, v)=\left(h^{1}\left(p^{1}\right), p^{1}\right)$. The singular heteroclinic solution has an internal layer based at $\mathcal{C} \cap \Gamma^{1}$. Moreover, asymptotic expansions of internal layer solutions can be obtained to any order of $\epsilon$. The critical eigenvalue can also be determined by the angle of intersection of $\mathcal{C}$ and $\Gamma^{1}$. This is most useful if $\mathcal{C}$ and $\Gamma^{1}$ has multiple intersection points, for it shows that generically the stability index of these mono-layered solutions changes alternatively. See the example in $\S 6.3$.

There is a close relation between our approach to the geometric singular perturbation theory. According to Fenichel [10], there exist smooth stable and unstable manifolds in $\mathbb{R}^{2 m+2 n}$ of the normally hyperbolic slow manifolds $u=h^{i}(v), i=0,1$. These manifolds admit smooth foliations by strongly stable and unstable fibers respectively. Let $\mathfrak{M}^{-}$be the union of unstable fibers passing through $(u, 0, v, w)$ with 
$u=h^{0}(v),(v, w) \in W^{u}\left(\left(p^{0}, 0\right)\right)$, and let $\mathfrak{M}^{+}$be the union of stable fibers passing through $(u, 0, v, w)$ with $u=h^{1}(v),(v, w) \in W^{s}\left(\left(p^{1}, 0\right)\right)$. Using the geometric singular perturbation theory, if $\mathfrak{M}^{-}$intersects transversely with $\mathfrak{M}^{+}$at $\epsilon=0$, then they also do so at small $\epsilon$. The internal layer solution is determined by this intersection.

It can be shown that the transverse intersection of $\mathfrak{M}^{-}$and $\mathfrak{M}^{+}$is equivalent to the condition $\mathcal{C} \pitchfork \Gamma^{1}$. Details are left to the readers. We have found a simple way to check Fenichel's transversal condition in $\mathbb{R}^{2 m+2 n}$ by reducing it to a lower dimensional space $\mathbb{R}^{2 n}$.

Suitable changes can also be made for the case of a singular traveling wave solution by included the wave speed as a phase variable.

Let us return to the original boundary value problem with boundary conditions at $x=0,1$. Again, the slow manifolds are normally hyperbolic. Let $\mathfrak{M}^{-}$be the union of strongly unstable fibers passing through $(u, 0, v, w, x)$ with $u=h^{0}(v),(v, w, x) \in$ $\mathcal{M}_{-}$, and let $\mathfrak{M}^{+}$be the union of strongly stable fibers passing through $(u, 0, v, w, x)$ with $u=h^{1}(v),(v, w, x) \in \mathcal{M}_{+}$. We prove that $\mathcal{C} \pitchfork \Gamma^{1}$ is equivalent to the transversal intersection of $\mathfrak{M}^{-}$and $\mathfrak{M}^{+}$as follows.

Let us write $u_{\xi \xi}+f(u, v)=0$ into a system $u_{\xi}=\hat{u}, \hat{u}_{\xi}+f(u, v)=0$. At the singular limit $\epsilon=0$, we pick a point $p=(u, \hat{u}, v, w, x) \in \mathfrak{M}^{-} \cap \mathfrak{M}^{+}$where $\wp=(v, w, x)$ is on $\mathcal{M}_{-} \cap \mathcal{M}_{+}$and $(u, \hat{u})=(q(0), \dot{q}(0))$ is on the heteroclinic solution $(q, \dot{q})$ connecting $h^{0}(v)$ to $h^{1}(v)$. Let

$$
(\Delta u, \Delta \hat{u}, \Delta v, \Delta w, \Delta x) \in T_{p} \mathfrak{M}^{-} \cap T_{p} \mathfrak{M}^{+} .
$$

Then $(\Delta v, \Delta w, \Delta x) \in T_{\wp} \mathcal{C}$. On the other hand, since moving along $(\Delta v, \Delta w, \Delta x)$ does not break the heteroclinic solution, we must have $(\Delta v, \Delta w, \Delta x) \in T_{\wp} \Gamma^{1}$.

If $\Gamma^{1} \pitchfork \mathcal{C}$, from the above argument, we have $(\Delta v, \Delta w, \Delta x)=0$, and the tangent vector $(\Delta u, \Delta \hat{u}, 0,0,0)$ is on $T_{\wp} W^{u} \cap T_{\wp} W^{s}$. But the strongly unstable fiber $W^{u}(\wp)$ has a one-dimensional intersection with the strongly stable fiber $W^{s}(\wp)$. This shows that $(\Delta u, \Delta \hat{u})=C(\dot{q}(0), \ddot{q}(0))$ where $C$ is a scalar, and $T_{p} \mathfrak{M}^{-} \cap T_{p} \mathfrak{M}^{+}$is onedimensional. Since $\operatorname{dim} T_{p} \mathfrak{M}^{-}=\operatorname{dim} T_{p} \mathfrak{M}^{+}=2 n+1+m$ and the intersection occurs in a $(2 n+2 m+1)$-dimensional space, thus $\mathfrak{M}^{-} \pitchfork \mathfrak{M}^{+}$. The converse is also true.

\section{ExAmples}

6.1. A $x$-dependent system. When the matrix coupling $A$ is diagonal, then there is no coupling among the internal layers through the slow field up to $O(\epsilon)$, and the $r$ eigenvalues, $\lambda_{1}$, are determined locally layer by layer. This happens if the jumps $\mathcal{M}^{i} \stackrel{\text { def }}{=} g\left(q^{i}(-\infty), \bar{v}^{i}\right)-g\left(q^{i}(\infty), \bar{v}^{i}\right)=0$ for $1 \leq i \leq r$.

As a special case, consider the following $x$ dependent system

$$
\begin{aligned}
& \epsilon^{2} u_{x x}+f(u, x)=0, \quad u \in \mathbb{R}^{m}, 0<x<1, \\
& u_{x}=0, \quad x=0,1 .
\end{aligned}
$$

Letting $v=x$, we have (1.4) with $g=0$, whence $\mathcal{M}^{i}=0$ for all $i$. Therefore, $\left(V_{c}^{i}, W_{c}^{i}\right)=0$ and $V_{1}^{R}=0$. See (4.18). The coupling matrix has the simplest form

$$
A=-\operatorname{diag}\left(\mathbf{n}^{i} \cdot w_{0}^{R}\left(x_{0}^{i}\right)\right)_{i=1}^{r} .
$$

Using $w_{0}^{R}=v_{0 x}^{R}=1$ and (3.5), we have the $r$ eigenvalues

$$
\lambda_{1}=-\left\langle\psi^{i}, f_{x}\left(q^{i}(\xi), x_{0}^{i}\right)\right\rangle, \quad 1 \leq i \leq r .
$$


The above formula for $\lambda_{1}$ is valid when $u$ and $f$ are in $\mathbb{R}^{m}, m \geq 1$. For scalar equations, observing that the linear equation $U_{\xi \xi}+f_{u}\left(\dot{q}^{i}(\xi), x_{0}^{i}\right) U=0$ is self adjoint in $L^{2}(\mathbb{R})$, we must have $\psi^{i}=\dot{q}^{i} /\left|\dot{q}^{i}\right|^{2}$. See (H2). Therefore,

$$
\begin{aligned}
\lambda_{1} & =-\left|\dot{q}^{i}\right|^{-2} \int_{-\infty}^{\infty} \dot{q}^{i}(\xi) f_{x}\left(q^{i}(\xi), x_{0}^{i}\right) d \xi \\
& =-\left|\dot{q}^{i}\right|^{-2} \frac{d}{d x} \int_{q^{i}(-\infty)}^{q^{i}(\infty)} f\left(u, x_{0}^{i}\right) d u .
\end{aligned}
$$

Following Fife [12, let $J^{i}(x)=\int_{h^{i-1}(x)}^{h^{i}(x)} f(u, x) d u$. Then

$$
\lambda_{1}=-\left|\dot{q}^{i}\right|^{-2} \frac{d}{d x} J^{i}\left(x_{0}^{i}\right), \quad 1 \leq i \leq r .
$$

The existence of a heteroclinic solution at $x^{i}$ is equivalent to $J^{i}\left(x_{0}^{i}\right)=0$ (equal area rule), while (H3) is equivalent to $\frac{d}{d x} J^{i}\left(x_{0}^{i}\right) \neq 0$.

In the original AMP model, $f(u, x)=\left(1-u^{2}\right)(u-a(x))$. If $q^{i}$ connects $u=-1$ to $u=1$, then $J^{i}(x)=-\frac{4}{3} a(x)$. Thus $J^{i}\left(x_{0}^{i}\right)=0 \Leftrightarrow a\left(x_{0}^{i}\right)=0$, and $\lambda_{1}=$ $\frac{4}{3}\left|\dot{q}^{i}\right|^{-2} a^{\prime}\left(x_{0}^{i}\right)$. It is known that $q^{i}(\xi)=\tanh \left(\frac{\xi}{\sqrt{2}}\right)$ and $\left|\dot{q}^{i}\right|_{L^{2}}^{2}=\frac{2 \sqrt{2}}{3}$. Therefore, $\lambda_{1}=\sqrt{2} a^{\prime}\left(x_{0}^{i}\right)$.

Similarly, for the internal layer jumping from near $u=1$ to $u=-1$, we can show that $\lambda_{1}=-\sqrt{2} a^{\prime}\left(x_{0}^{i}\right)$.

We summarize the results in the following

Theorem 6.1. For the $x$-dependent system, $A$ is diagonal, with

$$
\lambda_{1}=-\left\langle\psi^{i}, f_{x}\left(q^{i}(\xi), x_{0}^{i}\right)\right\rangle, \quad 1 \leq i \leq r .
$$

In particular, for the AMP model $\lambda_{1}=\operatorname{sign}\left\{\dot{q}^{i}\right\} \sqrt{2} a^{\prime}\left(x_{0}^{i}\right)$.

The stability index of the multi-layered solution derived from above agrees with the result in [1].

6.2. Coupled Ginzburg-Landau equations. Consider (1.1) with $f(u, v)=u-$ $u^{3}-\frac{1}{3} v, g(u, v)=\sigma\left(v-v^{3}\right)$ and $u_{x}=v_{x}=0$ at $x=0,1$. The stationary solution of this system is a pair of Duffing oscillators with a unilateral coupling in the fast equation. We will show that Lemma $\mathrm{A}$ in $\S 1$ is not satisfied in this example. This example is highly special since the slow equation does not contain $u$. At the end of this subsection, we will give another example where coupling terms appear in both equations.

As in the examples in $\S 1, f(u, v)=0$ has three branches of solution manifolds $u=h_{0}(v)$ and $u=h_{ \pm}(v)$. At $\bar{v}=0$, (1.5) has a heteroclinic loop, $q(\xi)$ and $q(-\xi)$, connecting $u=h_{-}(0)$ and $u=h_{+}(0)$. Write the second equation of (1.4) as a system

$$
\begin{aligned}
v_{x} & =w, \\
w_{x} & =-\sigma\left(v-v^{3}\right) .
\end{aligned}
$$

For any constant $\sigma>0$, (6.1) has three equilibria $(v, w)=(0,0)$ and $( \pm 1,0)$ and has two heteroclinic orbits connecting the hyperbolic equilibria $( \pm 1,0)$. Notice that the interval $[-1,1]$ is contained in the domains of $h_{ \pm}(v)$. The region bounded by the heteroclinic loop is filled up with periodic solutions that surround the center $(0,0)$. For every point $(\eta, 0), 0<\eta<1$, on the $v$ axis, there passes a unique periodic 
orbit whose period will be denoted $d(\eta)$. Using an elliptic integral one can show that $d^{\prime}(\eta)>0$ and there exist one sided limits $d\left(0^{+}\right)=\frac{2 \pi}{\sqrt{\sigma}}$ and $d(1)=\infty$. For any $m \in \mathbb{N}^{+}$, let $\sigma$ be sufficiently large so that $\frac{2 \pi}{\sqrt{\sigma}}<\frac{2}{m}$. Fix that $\sigma$. It is clear from the above that there exists a unique $0<\eta_{0}<1$ such that $d\left(\eta_{0}\right)=2 / m$. Let $(v(x), w(x))$ be the period $2 / m$ solution that satisfies $v(0)=\eta_{0}, w(0)=0$. Let $\left(v_{0}^{R}(x), w_{0}^{R}(x)\right)$ be the restriction of $(v(x), w(x))$ to $x \in[0,1] . v_{0}^{R}$ satisfies the following.

$$
\begin{aligned}
& v_{x}(\ell / m)=0, \quad \ell=0,1, \ldots, m, \\
& v(\ell / m)=(-1)^{\ell} \eta_{0}, \quad \ell=0,1, \ldots, m, \\
& v\left(\frac{1}{2 m}+\frac{\ell}{m}\right)=0, \quad \ell=0,1, \ldots, m-1 .
\end{aligned}
$$

Let $\left\{x^{1}<x^{2}<\cdots<x^{r}\right\}$ be a subset of $\left\{\frac{1}{2 m}+\frac{\ell}{m}: \ell=0,1, \ldots, m-1\right\}$. It is clear that $v_{0}^{R}\left(x^{i}\right)=0,1 \leq i \leq r$. Let $x^{0}=0$ and $x^{r+1}=1$. For $x \in\left(x^{i-1}, x^{i}\right)$, define $u_{0}^{R}(x)=h_{+}\left(v_{0}^{R}(x)\right)$ if $i$ is odd, and $u_{0}^{R}(x)=h_{-}\left(v_{0}^{R}(x)\right)$ if $i$ is even. The function $\left(u_{0}^{R}, v_{0}^{R}\right)$ is the 0th order expansion of an $r$-layered solution in regular layers. Let

$$
u_{0}^{S i}(\xi)= \begin{cases}q^{i}(\xi) \equiv q(-\xi), & \text { if } i \text { is odd } \\ q^{i}(\xi) \equiv q(\xi), & \text { if } i \text { is even }\end{cases}
$$

Let $v_{0}^{S i}(\xi)=0$. The union of $\left(u_{0}^{R}(x), v_{0}^{R}(x)\right)$ in regular layers $\left(x^{i-1}, x^{i}\right), i=$ $1,2, \ldots, r+1$, and $\left(u^{S i}(\xi), v_{0}^{S i}(\xi)\right)$ in singular layers at $x^{i}, i=1,2, \ldots, r$, is a singular internal layer solution. We can verify that Hypotheses (H1)-(H7) are satisfied by this solution.

It is trivial to verify (H1)-(H3) since the $u$-equation is the same as the activatorinhibitor model in $\S 1$. The Transversality Hypothesis in $\S 1$ is satisfied since the fast jump surface $\Gamma^{i}=\{(v, w, x): v=0\}$ is transverse to the flow at each $x^{i}$, due to $v_{0 x}^{R}\left(x^{i}\right) \neq 0$. From the transversality hypothesis, (H4) and (H5) are satisfied. We only need to prove $(\mathrm{H} 6)$ and $(\mathrm{H} 7)$. Let $\mathcal{S}_{0}=\{(v, w, x): x=0, w=0\}, \mathcal{S}_{1}=$ $\{(v, w, x): x=1, w=0\}$ as in $\S 3$.

Let $\Phi(x)\left(v_{0}, w_{0}\right)$ be the solution map for 6.1) with $\Phi(0)\left(v_{0}, w_{0}\right)=\left(v_{0}, w_{0}\right)$. Assume that $v_{0}^{R}(x)$ consists of $m$ monotonic paths with $m$ being even. (The case $m$ is odd can be considered similarly.) Let $\left(v_{0}^{R}(0), w_{0}^{R}(0)\right)=\left(\eta_{0}, 0\right)$. Then $\left(v_{0}^{R}(1), w_{0}^{R}(1)\right)=\left(\eta_{0}, 0\right)$ since $m$ is even. Let $\Delta \eta$ be a small variation of $\eta_{0}$. The periodic solution with the initial data $\left(\eta_{0}+\Delta \eta, 0\right)$ has the period $d\left(\eta_{0}+\Delta \eta\right)=$ $2(1+\Delta x) / m$ where $\Delta x$ is small. This leads to $\Phi(1+\Delta x)\left(\eta_{0}+\Delta \eta, 0\right)=\left(\eta_{0}+\Delta \eta, 0\right)$. Since $d^{\prime}(\eta)>0$, we have

$$
\frac{d \Delta x}{d \Delta \eta}>0
$$

A tangent vector on $\left(\mathcal{P}^{r} \ldots \mathcal{P}^{1} \mathcal{P}^{0}\right) \mathcal{S}_{0}$ can be obtained by taking the limit as $\Delta \eta \rightarrow 0$ on the following vector

$$
\begin{aligned}
\frac{\Phi(1)\left(\eta_{0}+\Delta \eta, 0\right)-\Phi(1)\left(\eta_{0}, 0\right)}{\Delta \eta} & =\frac{\Phi(1)\left(\eta_{0}+\Delta \eta, 0\right)-\Phi(1+\Delta x)\left(\eta_{0}+\Delta \eta, 0\right)}{\Delta \eta} \\
& +\frac{\Phi(1+\Delta x)\left(\eta_{0}+\Delta \eta, 0\right)-\Phi(1)\left(\eta_{0}, 0\right)}{\Delta \eta}
\end{aligned}
$$

As $\Delta \eta \rightarrow 0$, the first quotient in the right hand side has the limit

$$
\frac{d \Delta x}{d \Delta \eta}\left(w_{0}^{R}(1),-\sigma\left(v_{0}^{R}(1)-\left(v_{0}^{R}(1)\right)^{3}\right)\right)
$$


due to equation (6.1), and the second has the limit $(1,0)$ since

$$
\Phi(1+\Delta x)\left(\eta_{0}+\Delta \eta, 0\right)=\left(\eta_{0}+\Delta \eta, 0\right)
$$

and

$$
\Phi(1)\left(\eta_{0}, 0\right)=\left(\eta_{0}, 0\right) .
$$

Thus, $\left(1,-\frac{d \Delta x}{d \Delta \eta} \sigma\left(v_{0}^{R}(1)-\left(v_{0}^{R}(1)\right)^{3}\right)\right)$ is a tangent vector on $\left(\mathcal{P}^{r} \ldots \mathcal{P}^{1} \mathcal{P}^{0}\right) \mathcal{S}_{0}$. By (6.3) and $-\sigma\left(v_{0}^{R}(1)-\left(v_{0}^{R}(1)\right)^{3}\right) \neq 0$, the tangent vector is not on $T \mathcal{S}_{1}$. This proves both (H6) and (H7).

The results of $\S 3$ and $\S 4$ can be used on our system since $(\mathrm{H} 1)-(\mathrm{H} 7)$ are valid. We conclude that there is a matched asymptotic expansion of internal layer solution $\left(\sum \epsilon^{j} u_{j}, \sum \epsilon^{j} v_{j}\right)$. Due to the special form of our system, $v=v_{0}^{R}$ is independent of $\epsilon$. There also exist asymptotic expansions of critical eigenvalues $\sum \epsilon^{j} \lambda_{j}$ and corresponding eigenfunctions $\left(\sum \epsilon^{j} U_{j}, \sum \epsilon^{j} V_{j}\right)$ both in internal and regular layers.

From (H7), we see that the eigenvalues for the problem $\lambda V=V_{x x}+g_{v}\left(v_{0}^{R}(x)\right) V$ is nonzero. Let $\lambda(\epsilon)$, be a critical eigenvalue for (1.10) and (1.11). Using $\lambda_{0}=0$, we infer that the eigenfunction $(U, V)$ satisfies $V \equiv 0$. Substituting into (1.10), the critical eigenvalue satisfies

$$
\lambda U=\epsilon^{2} U_{x x}+f_{u} U, \quad U_{x}(0)=U_{x}(1)=0 .
$$

From the above, the critical eigenvalue is precisely the eigenvalue of the operator $\epsilon^{2} D_{x x}+f_{u}$ in the function space $H_{N}^{2}(I)$, see [39]. Therefore, the system does not satisfy Lemma A.

However, using the method of $\S 4$, we can calculate expansions of $\lambda$ to any order of $\epsilon$. In particular, since $V_{1}^{R}=0$, the coupling matrix $A_{r \times r}$ is diagonal. From (4.20), the $i$ th critical eigenvalue satisfies $\lambda_{1}=-\mathbf{n}^{i} \cdot v_{0 x}^{R}\left(x^{i}\right)$, similar to the case in the AMP model.

We now briefly describe another example where both equations contain coupling terms. The example is adapted from the AMP model. Consider (1.1) again with $f(u, v)=\left(1-u^{2}\right)\left(u-\frac{1}{2} v\right), g(u, v)=\sigma\left(v-v^{3}\right)+\gamma u^{2} v$, and $u_{x}=v_{x}=0$ at $x=0,1$. The roots of $f(u, v)=0$ consist of three branches: $u=h_{ \pm}(v)$ and $u=h_{0}(v)$ where $h_{+}(v)=1, h_{-}(v)=-1$ and $h_{0}(v)=\frac{1}{2} v$. (1.5) has a heteroclinic loop $q(\xi)$ and $q(-\xi)$ connecting the equilibria $u= \pm 1$ if $\bar{v}=0$. In regular layers, inserting $u= \pm 1$ into the second equation of (1.4), we have a reduced system

$$
v_{x x}+\sigma\left(v-v^{3}\right)+\gamma v, \quad v_{x}(0)=v_{x}(1)=0 .
$$

For any $m \in \mathbb{N}^{+}$, as before, we can find $\sigma>0, \gamma>0$ so that the above has an oscillatory solution $v_{0}^{R}(x)$ that satisfies (6.2). For any $r \leq m$, a singular $r$-layered solution can be defined as in the previous example. One can verify that (H1)(H7) are satisfied, so the method of $\S 3$ and $\S 4$ can be used to obtain asymptotic expansions for internal layer solutions and critical eigenvalues and eigenfunctions. Since $g(1,0)=g(-1,0), \mathcal{M}^{i}=0$ for all $1 \leq i \leq r$, cf. (4.16). Therefore, the eigenfunction $(U, V)$ for a critical eigenvalue satisfies $V_{1}^{R}=0$. The coupling matrix is diagonal. The critical eigenvalues for (1.10), (1.11) and for the operator $\epsilon^{2} D_{x x}+f_{u}$ in $H_{N}^{2}(I)$ agree up to $\epsilon^{1}$.

6.3. Multiple existence of mono-layer solutions. This is the longest example and partially motivates the entire paper. As in the introduction, we consider a homotopy between the AMP and the NF types system. Assume that $a(x)=\sin (\omega x+b)$ and $\alpha \approx 1, \beta \approx 0$ so that the system can be treated as a 
perturbation to the NF type system. After rescaling, assume that $\alpha=1$ and $0 \leq \beta \leq \beta_{0}$ where $\beta_{0}>0$ is independent of $\epsilon$ and is to be determined in the sequel. Only mono-layer solutions will be considered. Our goal is to show that by choosing $(\beta, \omega, b)$, the system may have any prescribed number of mono-layer solutions. Moreover, some of these solutions can only be found by the new shooting method using pseudo-Poincaré mappings.

We will only consider mono-layers that jumps upwards, so the superscript $i=1$ which is used to index internal layers will be dropped. For convenience, let $\beta=\frac{k}{\omega}$ where $0 \leq k \leq \beta_{0} \omega$. We consider the following system.

$$
\begin{aligned}
u_{t} & =\epsilon^{2} u_{x x}+F\left(u, y+\frac{k}{\omega} \sin (\omega x+b)\right), & & 0<x<1, \\
y_{t} & =y_{x x}+\sigma G(u, y), & & u, y \in \mathbb{R}, \\
u_{x} & =y_{x}=0, & & x=0,1 .
\end{aligned}
$$

Let $v=(y, x)$. The stationary solutions of (6.4) satisfies

$$
\begin{aligned}
& 0=\epsilon^{2} u_{x x}+f(u, v), \\
& 0=v_{x x}+g(u, v), \\
& u_{x}=y_{x}=0, \quad x=0,1, \\
& x(0)=0, x(1)=1,
\end{aligned}
$$

where

$$
\begin{aligned}
& f(u, v)=F\left(u, y+\frac{k}{\omega} \sin (\omega x+b)\right), \\
& g(u, v)=\left(\begin{array}{c}
\sigma G(u, y) \\
0
\end{array}\right) .
\end{aligned}
$$

The above system is of the form (3.2).

The assumptions on $F$ and $G$ are listed in A1-A5 below. The nullclines of $F$ and $G$ are plotted in Figure 6.1. These assumptions are identical to those used in [39] and are qualitatively similar to the activator inhibitor model (1.1), (1.2).

A1. The nullcline of $F$ is sigmoidal and consists of three curves

$$
\begin{aligned}
R^{-} & =\left\{(u, y): u=h_{-}(y), y \in I_{-}\right\}, \\
R^{0} & =\left\{(u, y): u=h_{0}(y), y \in I_{0}\right\}, \\
R^{+} & =\left\{(u, y): u=h_{+}(y), y \in I_{+}\right\},
\end{aligned}
$$

where

$$
I_{-}=\left(y_{-}, \infty\right), \quad I_{0}=\left(y_{-}, y_{+}\right), \quad I_{+}=\left(-\infty, y_{+}\right) .
$$

A2. Define $J(y)=\int_{h_{-}(y)}^{h_{+}(y)} F(s, y) d s . J(y)$ has an isolated zero $\tilde{y} \in\left(y_{-}, y_{+}\right)$:

$$
J(\tilde{y})=0, \quad d J(\tilde{y}) / d y<0 .
$$

A3. $F_{u}<0$ on $R^{-}$and $R^{+}$.

A4. $G<0$ on $R^{-}$and $G>0$ on $R^{+} \cdot \frac{d}{d y} G\left(h_{ \pm}(y), y\right)<0$ for $y \in I_{ \pm}$. The latter is equivalent to $-G_{u} F_{u}^{-1} F_{y}+G_{y}<0$. 


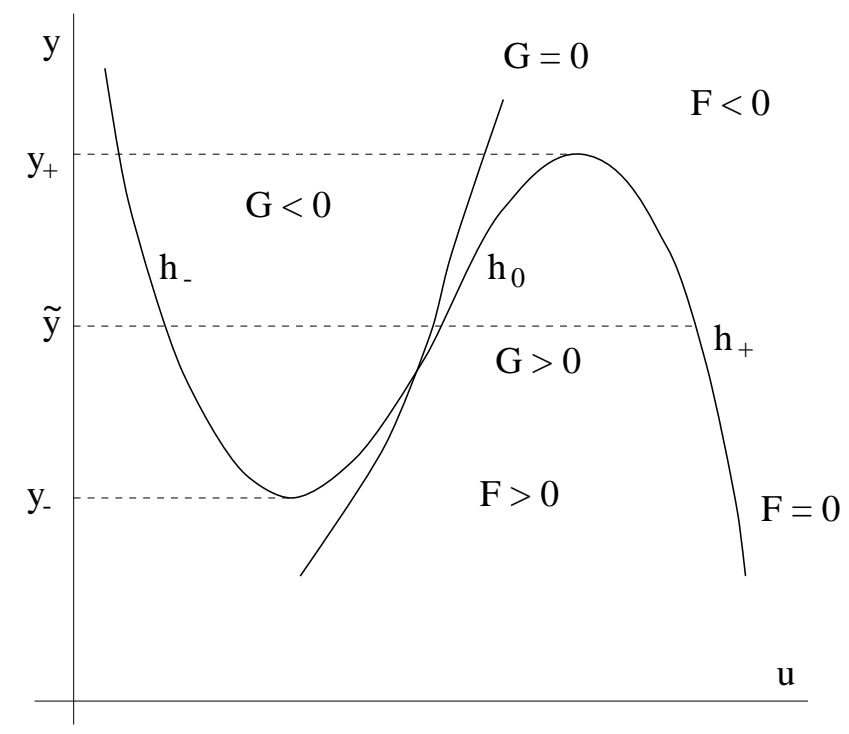

Figure 6.1. The nullclines of $F$ and $G$

A5. $\left.G_{y}\right|_{R^{ \pm}} \leq 0$.

To use the method in our paper, we verify (H1)-(H8). For a mono-layer solution, (H8) is always satisfied. We first verify that when $k=0$, the singular limit solution actually satisfy (H1)-(H7). We then use a perturbation method to show that (H1)(H7) are satisfied when $k / \omega$ is small and when certain conditions are posed on parameters $(k, \omega, b)$.

The unperturbed system: $k=0$. Under A1-A5, it is well known that there exist $\sigma_{0}, \epsilon_{0}>0$ such that (6.4) has a unique stationary mono-internal layer solution $(u(x, \epsilon), y(x, \epsilon))$ if $0<\sigma<\sigma_{0}$ and $0<\epsilon<\epsilon_{0}$ [12, 14, 23, 36. This solution jumps upwards from near $u=h_{-}(y)$ to near $u=h_{+}(y)$ at $x \approx x_{0}$. As $\epsilon \rightarrow 0$, this solution has a limit $\left(u_{0}^{R}(x), y_{0}^{R}(x)\right)$ in two regular layers separated by $x_{0} \in$ $(0,1)$. $\quad\left(u_{0}^{R}(x), y_{0}^{R}(x)\right)$ has a jump discontinuity at the internal layer $x_{0}$. Using a stretched variable $\xi=\left(x-x_{0}\right) / \epsilon$, there exists the limit in the internal layer $\left(u\left(x_{0}+\epsilon \xi, \epsilon\right), y\left(x_{0}+\epsilon \xi, \epsilon\right)\right) \rightarrow\left(u_{0}^{S}(\xi), y_{0}^{S}(\xi)\right)$ as $\epsilon \rightarrow 0$. The mono-layer solution $(u(x, \epsilon), y(x, \epsilon))$ and the limit in regular layers $\left(u_{0}^{R}, y_{0}^{R}\right)$ are plotted in Figure 6.2 In particular, the jump point $x_{0}$ satisfies $y_{0}^{R}\left(x_{0}\right)=\tilde{y}$ where $\tilde{y}$ as in A2, and $y_{0}^{R}$ is concave up for $x<x_{0}$ and concave down for $x>x_{0}$; and $y_{0 x}^{R}>0,0<x<1$.

There is a another mono-layer solution that jumps from near $u=h_{+}(y)$ to near $u=h_{-}(y)$. But this will not be used in this section. By mono-layer solution, we always mean the one that jumps upwards.

While the existence of the mono-internal layer solution is well known, the existence of matched expansions of this solution, or the existence of matched expansions of critical eigenvalue and eigenfunctions has not been proved before. To this end, we will verify $(\mathrm{H} 1)-(\mathrm{H} 7)$.

From A1, the slow manifolds are $R^{+}:=\left\{u=h_{+}(y)\right\}$ and $R^{-}:=\left\{u=h_{-}(y)\right\}$. From A3, $f_{u}<0$ on $R^{+} \cup R^{-}$. Thus (H1) is satisfied. It is clear that $v_{0}^{R}=\left(y_{0}^{R}, x\right)^{\tau}$ satisfies (3.4) with $x_{0}^{1}=x_{0}$ and $h^{0}=h_{-}, h^{1}=h_{+}$. At $x=x_{0}, \bar{v}=\left(\begin{array}{c}\tilde{y} \\ x_{0}\end{array}\right)$, the 


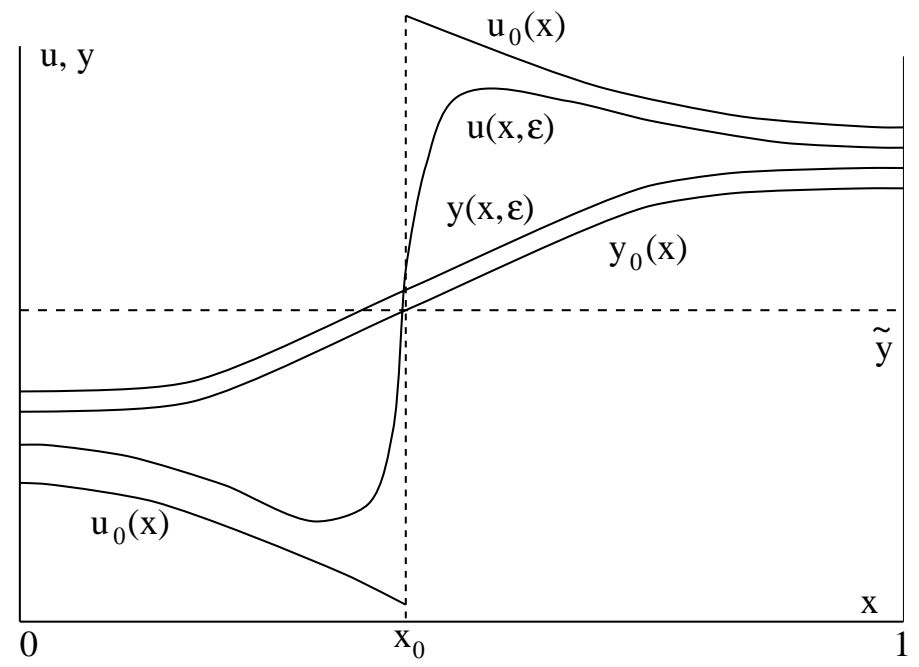

Figure 6.2. Mono-layer solutions and their singular limits

equation

$$
u_{\xi \xi}+f(u, \bar{v})=u_{\xi \xi}+F(u, \tilde{y})=0
$$

has a heteroclinic solution $q(\xi)$ due to the fact $J(\tilde{y})=0$, (the equal area rule, see A2). Thus the surface in (H2) is $\Sigma=\{(y, x) \mid y=\tilde{y}, x \in \mathbb{R}\}$.

The function $\dot{q}$ is clearly an eigenfunction corresponding to the eigenvalue $\lambda=0$. Using the fact

$$
U_{\xi \xi}+f_{u} U=0
$$

has exponential dichotomies on $\mathbb{R}^{-}$and $\mathbb{R}^{+}$, we see that $(q(0), \dot{q}(0))$ is in the intersection of the unstable subspace at $0-$ and stable subspace at $0+$; both are one dimensional. Thus, the eigenspace is spanned by $\dot{q}$. Equation (6.5) is self adjoint. Let $\psi=\dot{q} /\|\dot{q}\|_{L^{2}}$. Then $\langle\psi, \dot{q}\rangle=1$ and $\dot{q}$ is not in the range of the operator $\partial_{\xi \xi}+f_{u} \cdot I$. Condition (H2) is satisfied.

The normal of $\Sigma$ is

$$
\begin{aligned}
\mathbf{n} & =\int_{-\infty}^{\infty} f_{v}^{\tau}(q(\xi), \bar{v}), \psi(\xi) d \xi \\
& =\|\dot{q}\|^{-2} \int_{h_{-}(\tilde{y})}^{h_{+}(\tilde{y})} F_{v}(u, \tilde{y}) d u \\
& =\|\dot{q}\|^{-2} J_{v}(\tilde{y}) \\
& =\|\dot{q}\|^{-2} J^{\prime}(\tilde{y})\left(\begin{array}{l}
1 \\
0
\end{array}\right) .
\end{aligned}
$$

since $w_{0}^{R}\left(x_{0}\right)=\left(\begin{array}{c}y_{0 x}^{R}\left(x_{0}\right) \\ 1\end{array}\right)$, we have $\mathbf{n} \cdot w_{0}^{R}\left(x_{0}\right)=J^{\prime}(\tilde{y}) \cdot y_{0 x}^{R}\left(x_{0}\right) \neq 0$. Therefore, (H3) is satisfied. See A2. Also the flow is transverse to $\Sigma$.

The major job is to verify (H4)-(H7). We use a geometric method similar to that used in Theorem [5.3 Since the flow of the $x$ variable is trivial, it is reasonable 
to consider a reduced system that is equivalent to (3.6). (Equation (3.6) is 5dimensional.)

$$
\begin{aligned}
& d y / d x=z, \\
& d z / d x=-\sigma G\left(h\left(y+\frac{k}{\omega} \sin (\omega x+b)\right), y\right), \\
& d x / d x=1,
\end{aligned}
$$

where $k=0, h=h_{-}$if $x<x_{0}, h=h_{+}$if $x>x_{0}$. Obviously, $\left(y_{0}^{R}, z_{0}^{R}, x\right)$ where $z_{0}^{R}=y_{0 x}^{R}$ is a solution of (6.6). Let

$$
\begin{aligned}
& \bar{\Gamma}_{0}=\{(y, z, x) \mid x=0\}, \\
& \bar{\Gamma}_{1}=\{(y, z, x) \mid y=\tilde{y}\}, \\
& \bar{\Gamma}_{2}=\{(y, z, x) \mid x=1\}, \\
& \overline{\mathcal{S}}_{0}=\{(y, z, x) \mid x=0, z=0\}, \\
& \overline{\mathcal{S}}_{1}=\{(y, z, x) \mid x=1, z=0\}, \\
& \bar{\Pi}=\left\{(y, z, x) \mid x=x_{0}\right\} .
\end{aligned}
$$

Denote $\Phi_{-}$the solution map of (6.6) with $h=h_{-}$for all $0 \leq x \leq 1$. Denote $\Phi_{+}$ the solution map of (6.6) with $h=h_{+}$for all $0 \leq x \leq 1$. We first prove (H7).

Let

$$
\begin{aligned}
& \mathcal{M}_{-}=\bigcup\left\{\Phi_{-}(x, 0) \overline{\mathcal{S}}_{0} \mid 0 \leq x \leq 1\right\}, \\
& \mathcal{M}_{+}=\bigcup\left\{\Phi_{+}(x, 1) \overline{\mathcal{S}}_{1} \mid 0 \leq x \leq 1\right\} .
\end{aligned}
$$

Let $\mu_{0}=\mathcal{M}_{-} \cap \bar{\Pi}, \mu_{1}=\mathcal{M}_{-} \cap \bar{\Pi}$. Note that the matching point

$$
\wp=\left(y_{0}^{R}\left(x_{0}\right), z_{0}^{R}\left(x_{0}\right), x_{0}\right) \in \mu_{0} \cap \mu_{1} .
$$

Lemma 6.2. For the unperturbed system, $k=0,\left(H^{7}\right)$ is satisfied. Also, $\mu_{0} \pitchfork \mu_{1}$ at $\wp$ in $\bar{\Pi}$. See Figure 6.3 .

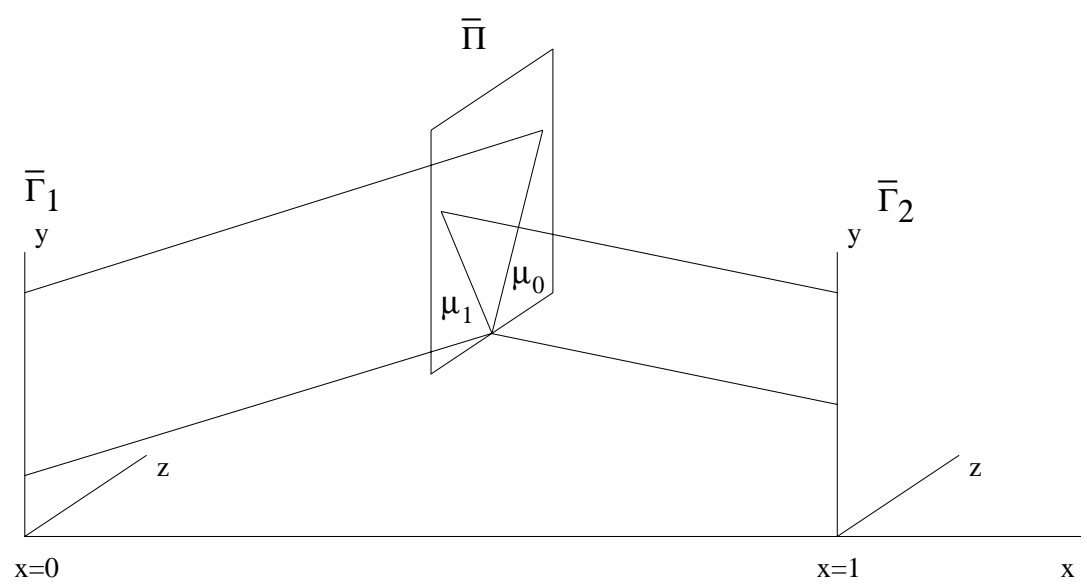

Figure 6.3. The transversal intersection of $\mu_{0}$ and $\mu_{1}$ 
Proof. We first prove (H7). From (H7), it is easy to see that $\mu_{0} \pitchfork \mu_{1}$ at $\wp$ in $\bar{\Pi}$.

Let $\left(Y^{-}, Z^{-}\right)$be the solution of the linear variational system of the of (6.6) around the 0th order expansion, with $k=0$,

$$
\begin{aligned}
& Y_{x}=Z, \\
& Z_{x}=\left(g_{u} f_{u}^{-1} f_{v}-g_{v}\right) Y, \\
& Y(0)=1, \\
& Z(0)=0,
\end{aligned}
$$

If we recall that $u^{R}=h\left(y^{R}\right)$ where $h=h_{-}$if $x<x_{0}$ and $h=h_{+}$if $x>x_{0}$, then using $g_{u} f_{u}^{-1} f_{v}-g_{v}=\sigma \frac{d}{d y} G(h(y), y)<0$ for $x \neq x_{0}$, it is easy to show that $Y^{-}\left(x_{0}\right)>1, Z^{-}\left(x_{0}\right)>0$. Similarly, the solution $\left(Y^{+}, Z^{+}\right)$of the linear variational system

$$
\begin{aligned}
& Y_{x}=Z, \\
& Z_{x}=\left(g_{u} f_{u}^{-1} f_{v}-g_{v}\right) Y, \\
& Y(1)=1, \\
& Z(1)=0,
\end{aligned}
$$

satisfies $Y^{+}\left(x_{0}\right)>1, Z^{+}\left(x_{0}\right)<0$.

Assume that (H7) is not satisfied for the mono-layer solution. Then there exists a nonzero $C^{1}$ solution $V$ to 4.12). Without loss of generality, assume that $V\left(x_{0}\right)>0$. Then there exist $\gamma_{1}, \gamma_{2}>0$ such that $V\left(x_{0}\right)=\gamma_{1} Y^{-}\left(x_{0}\right)=\gamma_{2} Y^{+}\left(x_{0}\right)$. However, we have a contradiction $V_{x}\left(x_{0}\right)=\gamma_{1} Z^{-}\left(x_{0}\right)>0$ and $V_{x}\left(x_{0}\right)=\gamma_{2} Z^{+}\left(x_{0}\right)<0$.

Since $\frac{d}{d x} y_{0}^{R}\left(x_{0}\right)>0, \bar{\Gamma}_{1}$ is a cross section of $\Phi_{-}$and $\Phi_{+}$, regular Poincaré mapping $\mathcal{P}^{i}: \bar{\Gamma}_{i} \rightarrow \bar{\Gamma}_{i+1}, i=0,1$, can be defined. Hypotheses (H4)-(H5) are clearly satisfied.

Lemma 6.2 implies that $\mathcal{M}_{-}$intersects $\mathcal{M}_{+}$transversely. The intersection is an one-dimensional curve $\mathcal{C}$ passing through $\wp$. As in $\S 5$, it is called a slow switching curve since the slow flow has to switch from $u=h_{-}(y)$ to $h_{+}(y)$ in order to satisfy boundary conditions for $y$ at $x=0,1$.

Let $\left(Y^{c}, Z^{c}\right)$ be a solution to the following linear system:

$$
\begin{aligned}
& d Y / d x=Z, \\
& d Z / d x=-\sigma \frac{d}{d y} G(h(y), y) Y, \\
& Z(0)=0, Z(1)=0, \\
& {[Y]\left(x_{0}\right)=0,} \\
& {[Z]\left(x_{0}\right)=\sigma\left(G\left(h_{-}(\tilde{y}), \tilde{y}\right)-G\left(h_{+}(\tilde{y}), \tilde{y}\right)\right) .}
\end{aligned}
$$

From Lemma 6.2, such a solution $\left(Y^{c}, Z^{c}\right)$ uniquely exists. Similar to $\S 5$, we can show that the vector

$$
\begin{aligned}
& \left(Y^{c}\left(x_{0}\right)-z_{0}^{R}\left(x_{0}\right), Z^{c}\left(x_{0}-\right)+\sigma G\left(h_{-}(\tilde{y}), \tilde{y}\right),-1\right) \\
& \quad=\left(Y^{c}\left(x_{0}\right)-z_{0}^{R}\left(x_{0}\right), Z^{c}\left(x_{0}+\right)+\sigma G\left(h_{+}(\tilde{y}), \tilde{y}\right),-1\right)
\end{aligned}
$$

is a tangent vector of $\mathcal{C}$ at $\left(y_{0}^{R}\left(x_{0}\right), z_{0}^{R}\left(x_{0}\right), x_{0}\right)$. Nishiura \& Fujii in [39] proved that $Y^{c}\left(x_{0}\right)-z_{0}^{R}\left(x_{0}\right)>0$. Thus, $\mathcal{C}$ intersects $\bar{\Gamma}_{1}$ transversely. Suppose that $\Phi_{-}$maps $\overline{\mathcal{S}}_{0}$ onto $\mathcal{S}_{-}$in $\bar{\Gamma}_{1}$ and $\Phi_{+}$maps $\overline{\mathcal{S}}_{1}$ onto $\mathcal{S}_{+}$in $\bar{\Gamma}_{1}$. It is now easy to show that

$$
\mathcal{S}_{-} \pitchfork \mathcal{S}_{+} \text {on } \bar{\Gamma}_{1}
$$

In fact, since $\mathcal{C}$ intersects $\bar{\Gamma}_{1}$ transversely, the tangent spaces of $\mathcal{M}_{ \pm}$have a common subspace $T_{\wp} \mathcal{C}$ which is not on $T_{\wp} \bar{\Gamma}_{1}$. If the tangent spaces of $\mathcal{S}_{-}$and $\mathcal{S}_{+}$coincide, 
then $T_{\wp} \mathcal{M}_{-}=T_{\wp} \mathcal{M}_{+}$. This is contradictory to $\mathcal{M}_{-} \pitchfork \mathcal{M}_{+}$. From here we deduce that (H6) is satisfied.

Perturbed internal layer solution: $k \neq 0$. We now show that $(\mathrm{H} 1)-(\mathrm{H} 7)$ are still valid if $k / \omega$ is small.

Assumption (H1) can be quickly verified by the Implicit Function Theorem. In fact, the slow manifolds are graphs of

$$
u=h_{ \pm}\left(y+\frac{k}{\omega} \sin (\omega x+b)\right) .
$$

In system (6.6), we now have $k \neq 0$ and $h=h_{-}$or $h_{+}$if $x<x^{\dagger}$, or $x>x^{\dagger}$ where the jump point $x^{\dagger}$ is part of the unknowns to be found here.

We use our geometric method to construct singular limit solutions of (6.6) and show that (H2)-(H7) are satisfied for these solutions. Since (6.6) is piecewise $C^{\infty}$, it is convenient to find the matching point $\wp=\left(y^{\dagger}, z^{\dagger}, x^{\dagger}\right)$ first. With $\wp$ as an initial point at $x=x^{\dagger}$, a solution can be obtained by solving (6.6) in $\left[0, x^{\dagger}\right]$ and $\left[x^{\dagger}, 1\right]$. Notice that when $k=0, \wp=\left(\tilde{y}, z_{0}^{R}\left(x_{0}\right), x_{0}\right)$.

First, due to the fact $J(\tilde{y})=0$, equation

$$
u_{\xi \xi}+F\left(u, y+\frac{k}{\omega} \sin (\omega x+b)\right)=0
$$

has a heteroclinic solution $q$ if $y+\frac{k}{\omega} \sin (\omega x+b)=\tilde{y}$. The fast jump surface for $k \neq 0$ is

$$
\bar{\Gamma}_{1}=\left\{(y, z, x) \mid y+\frac{k}{\omega} \sin (\omega x+b)=\tilde{y}\right\}
$$

The matching point must satisfy $\wp \in \bar{\Gamma}_{1}$. The surface $\bar{\Gamma}_{1}$ is plotted in Figure 6.4.

As in the case $k=0$, let $\Phi_{-}$and $\Phi_{+}$respectively be solution maps of (6.6) with $h=h_{-}$and $h=h_{+}$throughout $x \in[0,1]$. Define $\mathcal{M}_{-}$and $\mathcal{M}_{+}$as before.

The intersection of the three manifolds $\mathcal{M}_{ \pm}$and $\bar{\Gamma}_{1}$ determines the matching point $\wp$. Since it is difficult to study the intersection of $\bar{\Gamma}_{1}$ with $\mathcal{M}_{-}$or $\mathcal{M}_{+}$, we study the intersection of $\mathcal{M}_{-}$and $\mathcal{M}_{+}$first.

Lemma 6.3. The distances between $\mathcal{M}_{+}$and the corresponding ones with $k=0$ are $O\left(\frac{k}{\omega^{2}}\right)$ in the $C^{0}$ metric and are $O\left(\frac{k}{\omega}\right)$ in the $C^{1}$ metric. When $k \neq 0$ and $k / \omega$ is small, $\mathcal{M}_{-}$and $\mathcal{M}_{+}$intersect transversely. The intersection $\mathcal{C}=\mathcal{M}_{-} \cap \mathcal{M}_{+}$is ${ }_{a} C^{1}$ curve, and its distance from the one with $k=0$ is $O\left(k / \omega^{2}\right)$ in the $C^{0}$ metric. and is $O(k / \omega)$ in the $C^{1}$ metric.

Proof. With the initial data $(y, z, x)=(\eta, 0,0), \mathcal{M}_{-}$can be expressed as

$$
\mathcal{M}_{-}=\bigcup\left\{(y, z, x) \mid(y, z, x)=\Phi_{-}(x ; \eta, 0,0 ; k), \quad 0 \leq x \leq 1, \eta \in \mathbb{R}\right\},
$$

where $\Phi_{-}$is the solution map of $(6.6)$ with $h=h_{-}$. Also, $\frac{\partial \Phi_{-}}{\partial k}$ satisfies the linear variational system

$$
\begin{aligned}
& \left(y_{k}\right)^{\prime}=z_{k}, \\
& \left(z_{k}\right)^{\prime}=-\sigma \frac{d}{d y} G\left(h\left(y+\frac{k}{\omega} \sin (\omega x+b)\right), y\right) y_{k}-\sigma \frac{\partial}{\partial u} G(h, y) h^{\prime} \frac{1}{\omega} \sin (\omega x+b), \\
& \left(x_{k}\right)^{\prime}=0 .
\end{aligned}
$$




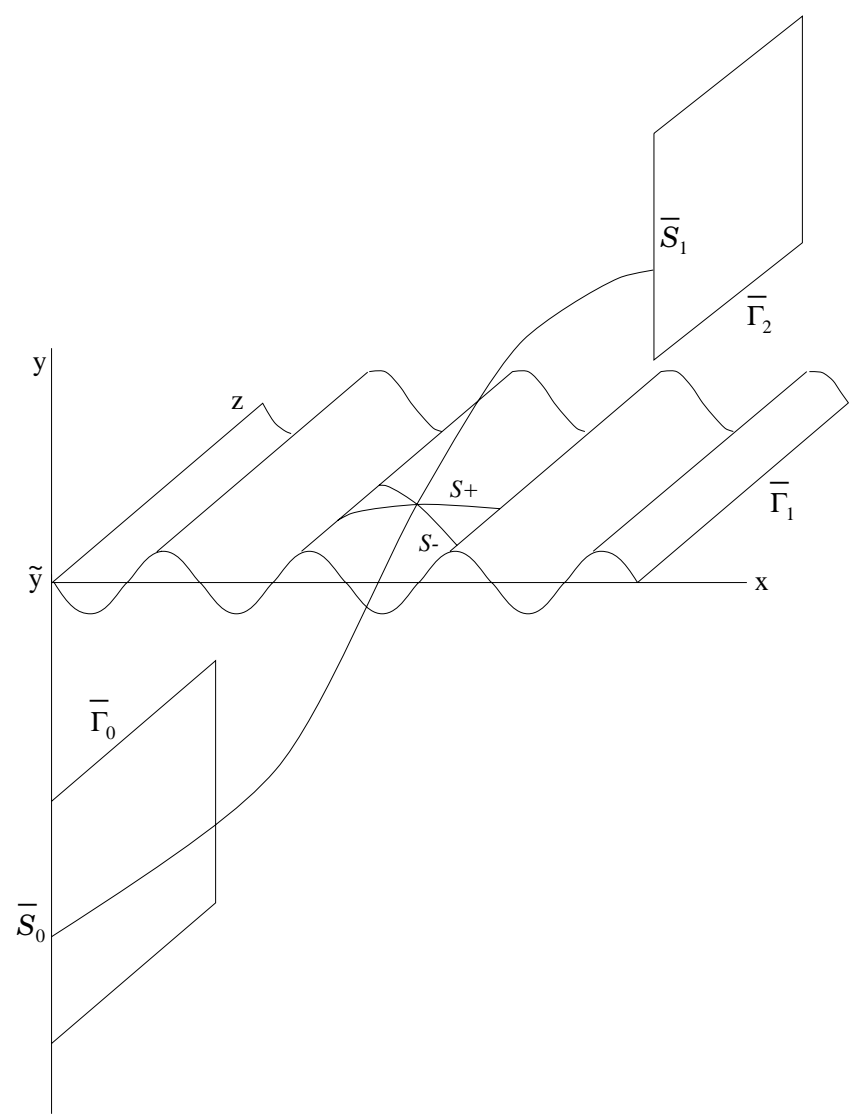

FIGURE 6.4. Poincaré mappings induced by the flow of (6.6)

The forcing term for (6.9) is of $O\left(\frac{1}{\omega}\right)$, thus, in general $\left(y_{k}, z_{k}, x_{k}\right)=O\left(\frac{1}{\omega}\right)$ only. However, since $\sin (\omega x+b)$ is fast oscillatory, using a standard method in the theory of averaging, we have

$$
\left(y_{k}, z_{k}, x_{k}\right)=O\left(\frac{1}{\omega^{2}}\right)
$$

This proves that

$$
\Phi_{-}(x ; \eta, 0,0 ; k)-\Phi_{-}(x ; \eta, 0,0 ; 0)=O\left(\frac{k}{\omega^{2}}\right) .
$$

Thus, the distance between $\mathcal{M}_{-}$and the one with $k=0$ is of $O\left(k / \omega^{2}\right)$ in the $C^{0}$ metric. Using the same method, one can show

$$
\frac{\partial}{\partial \eta}\left\{\Phi_{-}(x ; \eta, 0,0 ; k)-\Phi_{-}(x ; \eta, 0,0 ; 0)\right\}=O\left(\frac{k}{\omega}\right) .
$$

From the right hand side of system (6.9), we have

$$
\frac{\partial^{2}}{\partial k \partial x} \Phi_{-}(x ; \eta, 0,0 ; k)=O(1 / \omega) .
$$

Therefore, the distance between $\mathcal{M}_{-}$and the one with $k=0$ is of $O(k / \omega)$ in the $C^{1}$ metric. 
The statements about $\mathcal{M}_{+}$can be proved similarly.

The assertions concerning $\mathcal{C}$ can be proved using the implicit function theorem, or a contraction mapping principle and will not be given here.

When $k=0$,

$$
\left(Y^{c}\left(x_{0}\right)-z_{0}^{R}\left(x_{0}\right), Z^{c}\left(x_{0}-\right)+\sigma G\left(h_{-}(\tilde{y}), \tilde{y}\right),-1\right)
$$

is a tangent vector of $\mathcal{C}$ at $\wp$ with the $y$ component being positive. Therefore, locally the curve $\mathcal{C}$ can be expressed as

$$
x=x^{*}(y, b), \quad z=z^{*}(y, b), \quad \tilde{y}-T \leq y \leq \tilde{y}+T,
$$

where $x^{*}$ and $z^{*}$ are $C^{1}$ functions and $T>0$ is a constant.

Lemma 6.4. $\frac{\partial x^{*}}{\partial y}(\tilde{y}, b)=-\left(Y^{c}\left(x_{0}\right)-z_{0}^{R}\left(x_{0}\right)\right)^{-1}$ if $k=0$. If $k \neq 0$, then

$$
\begin{aligned}
& \frac{\partial}{\partial b} x^{*}(y, b)=O\left(k / \omega^{2}\right), \\
& \frac{\partial}{\partial b}\left(\frac{\partial x^{*}(y, b)}{\partial y}\right)=O(k / \omega) .
\end{aligned}
$$

Proof. The assertion for $k=0$ is obvious.

To prove the assertion about $\frac{\partial x^{*}}{\partial b}$, we can use a linear variational system to show that $\Phi_{ \pm}$is a $C^{1}$ function of $b$ and $\frac{\partial \Phi_{ \pm}}{\partial b}=O\left(k / \omega^{2}\right)$ in the $C^{0}$ metric and is of $O(k / \omega)$ in the $C^{1}$ metric. Similar to the proof of Lemma 6.3. the fast oscillatory property of $\sin (\omega x+b)$ is important in the proof.

Since $\mathcal{C}$ is the intersection of $\mathcal{M}_{ \pm}$, and since

$$
\mathcal{C}=\bigcup\left\{(y, z, x) \mid z=z^{*}(y, b), x=x^{*}(y, b)\right\}
$$

using the implicit function theorem, or contraction mapping principles, one can show that $z^{*}$ and $x^{*}$ are $C^{1}$ functions of $b$ and the desired estimates on $\frac{\partial}{\partial b} x^{*}$ and $\frac{\partial^{2}}{\partial b \partial y} x^{*}$.

Assume now $k \neq 0$ but $k / \omega$ small. The transversal intersection of $\mu_{0}$ and $\mu_{1}$ is still true after the small perturbation. Thus (H7) is satisfied. From Lemma 6.3 after the small perturbation, we still have $\mathcal{M}_{-} \pitchfork \mathcal{M}_{+}$. Locally, $\mathcal{C}$ is still of the form (6.10). Observe that the variation of $\bar{\Gamma}_{1}$ is $\pm \frac{k}{\omega}$ in the $y$ direction. If $\frac{k}{\omega}<T$, the intersection of $\mathcal{C}$ with $\bar{\Gamma}_{1}$ is nonempty. Each point in the intersection is a candidate for the switching point $\wp$. The question is whether $(\mathrm{H} 4)-(\mathrm{H} 6)$ are satisfied at the intersection. From Lemma 5.1, conditions (H4)-(H6) are satisfied if $\mathcal{C} \pitchfork \bar{\Gamma}_{1}$ at $\wp$.

Since $\bar{\Gamma}_{1}$ is flat in the $z$ direction, to study the transversality of the intersection of $\bar{\Gamma}_{1}$ and $\mathcal{C}$, it is convenient to project out their $z$ component. The images of the projection are $\bar{\Gamma}_{1} \rightarrow \Pi \Gamma_{1}$ and $\mathcal{C} \rightarrow \Pi \mathcal{C}$. Several possible intersections of $\mathcal{C}$ and $\bar{\Gamma}_{1}$ are depicted in Figure 6.5. The $z$ direction is not shown. It follows from Lemma 6.4 that if $T$ is sufficiently small and $\omega$ is sufficiently large, then $\frac{\partial}{\partial y} x^{*}(y, b)=$ $-\left(Y^{c}\left(x_{0}\right)-z_{0}^{R}\left(x_{0}\right)\right)^{-1}+O(T+k / \omega)<0$ for all $y \in(\tilde{y}-T, \tilde{y}+T)$. The nontransverse intersection of $\Pi \Gamma_{1}$ and $\Pi \mathcal{C}$ can occur at the part of $\Pi \Gamma_{1}$ that is decreasing. We now elaborate at this.

First assume that $k$ is sufficiently small so that

$$
\left|\frac{\partial}{\partial y} x^{*}(\tilde{y}, b)\right|_{k=0}<\frac{1}{k}
$$


The subscript $k=0$ indicates that the left hand side of (6.11) is evaluated at $k=0$. Using the estimate on the dependence of $\mathcal{C}$ on $k$ from Lemma 6.3, if $T$ and $k / \omega$ are sufficiently small, we have

$$
\left|\frac{\partial}{\partial y} x^{*}(y, b)\right|<\frac{1}{k}
$$

for all $\tilde{y}-T<y<\tilde{y}+T$. Since the maximum of the slope of $\Pi \Gamma_{1}$ is $k, \Pi \mathcal{C}$ intersects $\Pi \Gamma_{1}$ transversely at a unique point $\wp=\left(y^{\dagger}, z^{\dagger}, x^{\dagger}\right)$ for any $b \in \mathbb{R}$.

Next, assume that $k$ is sufficiently large so that

$$
\left|\frac{\partial}{\partial y} x^{*}(\tilde{y}, b)\right|_{k=0}>\frac{1}{k} .
$$

If $T$ and $k / \omega$ are sufficiently small, we have

$$
\left|\frac{\partial}{\partial y} x^{*}(y, b)\right|>\frac{1}{k}
$$

for all $\tilde{y}-T<y<\tilde{y}+T$.

The intersections of $\mathcal{C}$ and $\bar{\Gamma}_{1}$ correspond to solutions of the equation

$$
\frac{k}{\omega} \sin \left(\omega x^{*}(y, b)+b\right)+y=\tilde{y} .
$$

Let $\phi=\omega x^{*}(y, b)+b$. With $\Phi$ as a parameter on $\Pi \Gamma_{1}$, the intersections correspond to zeros of the function

$$
\mathfrak{E}(\phi, b) \stackrel{\text { def }}{=} \omega x^{*}\left(\tilde{y}-\frac{k}{\omega} \sin \phi, b\right)+b-\phi=0 .
$$

Once $\phi$ is found, the intersection is determined by

$$
x=\frac{\phi-b}{\omega}, \quad y=\tilde{y}-\frac{k}{\omega} \sin \phi .
$$

Since $\frac{\partial}{\partial b} x^{*}(y, b)=O\left(k / \omega^{2}\right)$, (Lemma 6.4), and $x^{*}(y, b)$ is a periodic function of $b$, we have $\frac{\partial \mathfrak{E}}{\partial b}>0$ and $\mathfrak{E} \rightarrow \pm \infty$ as $b \rightarrow \infty$. Therefore, if $\omega$ is sufficiently large so that $\frac{k}{\omega}<T$, then for each $\phi \in \mathbb{R}$, there exists a unique $b=b^{*}(\phi)$ such that $\mathfrak{E}=0$. Moreover, $b^{*}$ is a $C^{1}$ function of $\phi$ with

$$
\frac{\partial b^{*}}{\partial \phi}=\left(1+\omega \frac{\partial x^{*}}{\partial b}\right)^{-1}\left(1+k \frac{\partial x^{*}}{\partial y} \cos \phi\right) .
$$

Let $y_{0}^{R}$ be a solution constructed by such $\wp$. Condition $\mathcal{C} \pitchfork \bar{\Gamma}_{1}$ becomes $\partial b^{*} / \partial \phi \neq$ 0 , or equivalently,

$$
1+k \frac{\partial x^{*}}{\partial y} \cos \phi \neq 0 .
$$

Consider one period $\phi \in[-3 \pi / 2, \pi / 2]$ for the time being. For $\phi \in[-3 \pi / 2,-\pi / 2)$, $\cos \phi \leq 0,6.14$ is valid. The left hand side of (6.14) is positive if $\phi=-\pi / 2$, but is negative if $\phi=0$. Therefore, it is easy to see that there exist $\phi_{1} \in(-\pi / 2,0), \phi_{2} \in$ $(0, \pi / 2)$ such that

$$
1+k \frac{\partial x^{*}}{\partial y} \cos \phi_{j}=0, \quad j=1,2 .
$$

Using the fact that $\frac{\partial x^{*}}{\partial y}$ is almost a constant, it is easy to verify that $\phi_{1}, \phi_{2}$ are the only points in $[-3 \pi / 2, \pi / 2]$ that do not satisfy (6.14).

We summarize our results in the following theorem. 
Theorem 6.5. (1) Assume that $k$ is sufficiently small so that [6.11) is satisfied. Then there exists $\omega_{0}$, depending on $k$, such that if $\omega>\omega_{0}$, then for any $b \in \mathbb{R}$, there exists a unique singular limit solution $y_{0}^{R}$ that satisfies (H4)-(H6).

(2) Assume that $k$ is sufficiently large so that [6.12) is satisfied. Then there exists $\omega_{0}$, depending on $k$, such that if $\omega>\omega_{0}$, then the following is true: Assume that $\phi \neq \phi_{j}+2 \nu \pi, j=1,2, \nu \in \mathbb{Z}$. Let $b=b^{*}(\phi)$. Let the matching points $\wp=\left(y^{\dagger}, z^{\dagger}, x^{\dagger}\right)$ be determined by such $\phi$ and $b$. Then the singular limit solution $y_{0}^{R}$ constructed by $\wp$ satisfies Hypotheses (H4)-(H6). Moreover, for any $r>0$, there exists $k=k(r)$ such that if $\omega$ is chosen to be sufficiently large, then the number of solutions $y_{0}^{R}$ that satisfy $\left(\mathrm{H}_{4}\right)-(\mathrm{H} 6)$ is greater than $r$.
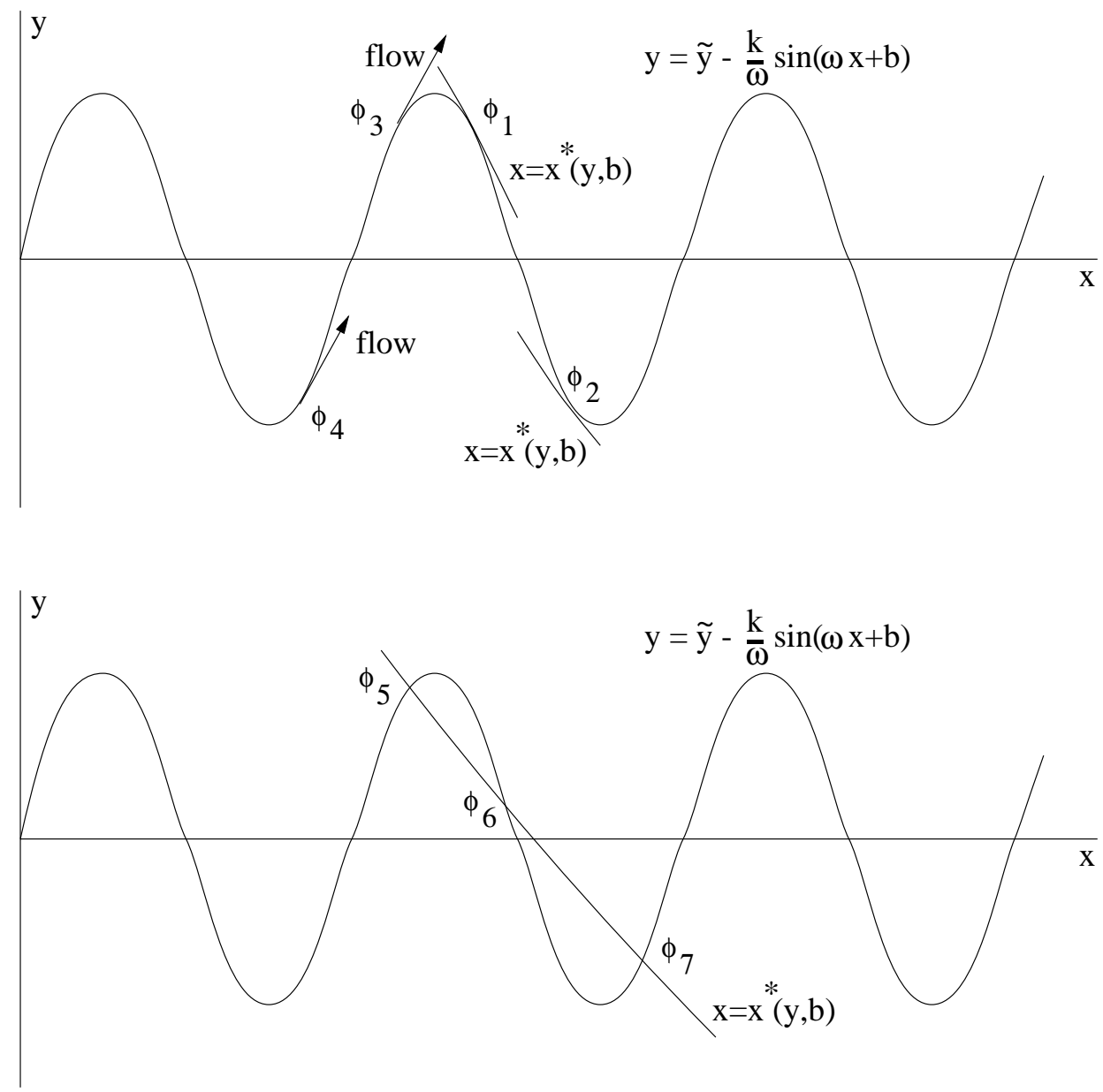

FiguRE 6.5. Several possible intersections of $\Pi \mathcal{C}$ and $\Pi \Gamma_{1}$ are depicted, not occurring simultaneously. (H4)-(H6) are not satisfied at the tangential intersections corresponding to $\phi_{1}, \phi_{2}$ but are satisfied at $\phi_{3}, \phi_{4}$ where the flow is tangent to $\Pi \Gamma_{1}$. The internal layer solution is stable at $\phi_{5}, \phi_{7}$ but unstable at $\phi_{6}$. 
Proof. When $\omega$ is sufficiently large, zooming in by a factor of $\omega$, the graph of $\Pi \mathcal{C}$ is approaching a straight line with constant slope. The proof of the last statement can be done by examining the graphs of the limiting straight line and $-k \sin \phi$.

The stability analysis of the perturbed internal layer solution is relatively easy once its existence is established. Since there is only one internal layer, the coupling matrix $A$ is a scalar. Recall that $v=(y, x), V=(Y, X)$ and $\psi=\dot{q} /\|\dot{q}\|_{L^{2}}^{2}$. From $\S 4$, the first two coefficients in the expansion of the critical eigenvalue $\lambda(\epsilon)$ are $\lambda_{0}=0$ and

$$
\begin{gathered}
\lambda_{1}=A=\mathbf{n} \cdot\left(V_{c}\left(x_{0}\right)-w_{0}^{R}\left(x_{0}\right)\right) . \\
\mathbf{n}=\int_{-\infty}^{\infty} f_{v}^{\tau}(q(\xi), \bar{v}) \psi(\xi) d \xi \\
=\|\dot{q}\|^{-2} \int_{h_{-}\left(y+\frac{k}{\omega} \sin (\omega x+b)\right)}^{h_{+}\left(y+\frac{k}{\omega} \sin (\omega x+b)\right)} F_{v}\left(u, y+\frac{k}{\omega} \sin (\omega x+b)\right) d u \\
=\|\dot{q}\|^{-2} J_{v}\left(y+\frac{k}{\omega} \sin (\omega x+b)\right) \\
=\|\dot{q}\|^{-2} J^{\prime}(\tilde{y})(1, k \cos (\omega x+b))^{\tau} .
\end{gathered}
$$

The basis solution $V_{c}=(Y, X)$ satisfies

$$
\begin{aligned}
& Y_{x x}-\sigma\left(G_{u} F_{u}^{-1} F_{v}-G_{v}\right)(Y, X)^{\tau}=0, \\
& X_{x x}=0, \\
& {[Y]\left(x_{0}\right)=[X]\left(x_{0}\right)=\left[X_{x}\right]\left(x_{0}\right)=0,} \\
& {\left[Y_{x}\right]\left(x_{0}\right)=\sigma\left(G\left(h_{-}(\tilde{y}), \tilde{y}\right)-G\left(h_{+}(\tilde{y}), \tilde{y}\right)\right),} \\
& X(0)=X(1)=0, \\
& Y_{x}(0)=Y_{x}(1)=0 .
\end{aligned}
$$

It follows that $X=0$. Then the equation for $Y$ simplifies to [6.8). Thus $Y=Y^{c}$. Since $w_{0}^{R}=\left(z_{0}^{R}, 1\right), V_{c}=\left(Y^{c}, 0\right)$,

$$
\begin{aligned}
\lambda_{1} & =\|\dot{q}\|^{-2} J^{\prime}(\tilde{y})\left(Y^{c}(x)-z_{0}^{R}(x)+X k \cos (\omega x+b)-k \cos (\omega x+b)\right) \\
& =\|\dot{q}\|^{-2} J^{\prime}(\tilde{y})\left(Y^{c}(x)-z_{0}^{R}(x)-k \cos \phi\right),
\end{aligned}
$$

where $x=x^{\dagger}$.

Consider the case when $k$ is sufficiently small so that (6.11) is satisfied. Using Lemma 6.4, if $T$ is sufficiently small and $\omega$ is sufficiently large, we have $0<k<$ $Y^{c}(x)-z_{0}^{R}(x)$. The unique internal layer solution as in Theorem 6.5 satisfies $\lambda_{1}<0$, and hence is stable. In particular, this is true when $k=0$. This result is in agreement with Nishiura \& Fujii [39].

Consider the case when $k$ is sufficiently large so that (6.12) is satisfied. Using Lemma 6.4 again we have $Y^{c}(x)-z_{0}^{R}(x)<k$. The stability of the internal layer solution depends on the parameter $\phi$. If $\phi_{1}+2 \nu \pi<\phi<\phi_{2}+2 \nu \pi, \nu \in \mathbb{Z}$, the internal layer solution is unstable $\left(\lambda_{1}>0\right)$; If $\phi_{2}+2 \nu \pi<\phi<\phi_{1}+2(\nu+1) \pi$, then the solution is stable $\left(\lambda_{1}<0\right)$.

Theorem 6.6. (1) Assume that (6.11) is satisfied. Then there exists $\omega^{*}>0$ such that for each $b \in \mathbb{R}$, there exists a unique internal layer solution that is stable.

(2) Assume that (6.12) is satisfied, Then there exists $\omega^{*}>0$ such that if $\omega>\omega^{*}$, then the following is true. Let $b=b^{*}(\phi)$ where $\phi \neq \phi_{j}+2 \nu \pi, j=1,2, \nu \in \mathbb{Z}$. Let $y_{0}^{R}$ be determined by such $b$ and $\phi$. Then the internal layer solution corresponding 
to such $y_{0}^{R}$ is unstable if $\phi_{1}+2 \nu \pi<\phi<\phi_{2}+2 \nu \pi, \nu \in \mathbb{Z}$, otherwise the solution is stable $\left(\lambda_{1}<0\right)$.

Remark. (i) Our analysis also suggests that when $b=b^{*}\left(\phi_{j}\right)+2 \pi \nu, j=1,2$, the intersection of $\mathcal{C}$ and $\bar{\Gamma}_{1}$ is nontransversal, and saddle-node type bifurcations may occur. The analysis of bifurcations caused by moving $b$ through critical values is completed in 18 .

(ii) Recall that $\left(Y^{c}(x)-z_{0}^{R}(x), \cdots,-1\right)$ is a tangent vector of $\mathcal{C}$. If $\mathcal{C}$ is oriented with the positive direction pointing to the decreasing of $x$, then $\lambda_{1}<0$ if $\mathcal{C}$ passes through $\bar{\Gamma}_{1}$ from below; $\lambda_{1}>0$ if $\mathcal{C}$ passes through $\bar{\Gamma}_{1}$ from above. This interpretation agrees with Theorem 5.3

(iii) The smallness of $k / \omega$ is only used to ensure that the shape of the slow switching curve $\mathcal{C}$ is computable by a perturbation method. For a general system, this curve can be obtained by numerically computing the intersection of $\mathcal{M}_{ \pm}$. Conceivably, we may find points where $\mathcal{C} \pitchfork \bar{\Gamma}^{1}$ is even when the oscillatory perturbation is not small. The break of stability and the bifurcation of internal layer solutions may occur in much general systems.

(iv) From Figure 6.5, we see that at the intersections corresponding to $\phi=\phi_{3}, \phi_{4}$, the flow of (6.6) is tangent to $\bar{\Gamma}_{1}$, however, the internal layer solution is structurally stable due to $\mathcal{C} \pitchfork \bar{\Gamma}_{1}$. These solutions will be missed if one insists that the flow must be transverse to $\bar{\Gamma}_{1}$.

\section{Final Remarks and Stability of internal Layer SOlutions}

1. Our methods of constructing asymptotic series for the internal layer solutions and the critical eigenvalue-eigenfunctions are actually related, although one uses the pseudo-Poincaré mapping or the (BVPIC), the other uses the coupling matrix (SLEP matrix). An intuitive reason is that the unknown shift $\left\{\Delta x^{i}\right\}$ in the (BVPIC) can also be formulated by adding multiples of $\dot{q}^{i}$ in the $i$ th internal layer as in the coupling matrix. The following lemma asserts that asymptotic expansions for internal layer solutions can be obtained if the coupling matrix is nonsingular:

Lemma 7.1. If in addition to (H4)-(H6) as in Lemma 3.2, condition (H7) is also valid, then (BVPIC) has a unique solution if and only if the coupling matrix is nonsingular.

Proof. Let $V_{c}^{i}$ and $\mathcal{M}^{i}$ be as in (4.17). Define $\bar{V}:=\sum_{1}^{r} V_{c}^{i} \Delta x^{i}$ which satisfies the first two equations in (BVPIC) with zero right sides, and

$$
\begin{aligned}
{[\bar{V}]\left(x_{0}^{i}\right) } & =0, \\
{\left[\bar{V}_{x}\right]\left(x_{0}^{i}\right) } & =\mathcal{M}^{i} \Delta x^{i}=\Delta x^{i}\left[w_{0 x}^{R}\right]\left(x_{0}^{i}\right), \quad 1 \leq i \leq r .
\end{aligned}
$$

Let $\tilde{V}=V+\bar{V}, V$ as in the (BVPIC), then

$$
\begin{aligned}
& \tilde{V}_{x x}-\left(g_{u} f_{u}^{-1} f_{v}-g_{v}\right) \tilde{V}=E_{1}, \\
& A_{j} \tilde{V}_{x}(j)+B_{j} \tilde{V}(j)=E_{2 j}, \quad j=0,1, \\
& {[\tilde{V}]\left(x_{0}^{i}\right)=E_{4}^{i},} \\
& {\left[\tilde{V}_{x}\right]\left(x_{0}^{i}\right)=E_{5}^{i} .}
\end{aligned}
$$

According to Lemma 4.1 the above has a unique solution $\tilde{V}$. Substituting $V=$ $\tilde{V}-\bar{V}$ into the third equation of (BVPIC), $\mathbf{n}^{i} \cdot\left(w_{0}^{R}\left(x_{0}^{i}\right) \Delta x^{i}+V\left(x_{0}^{i}+\right)\right)=E_{3}^{i}$, we 
have

$$
\mathbf{n}^{i} \cdot\left(w_{0}^{R}\left(x_{0}^{i}\right) \Delta x^{i}-\sum_{\ell=1}^{r} V_{c}^{\ell} \Delta x^{\ell}\right)=E_{3}^{i}-\mathbf{n}^{i} \tilde{V}\left(x_{0}^{i}+\right) .
$$

The linear system for $\Delta x^{i}$ has a unique solution if the coefficient matrix, the negation of the coupling matrix, is nonsingular.

2. The name "critical eigenvalue" used in this paper is not precise. Following Nishiura and Fujii, we have only considered critical eigenvalues whose eigenfunction $(U, V)$ has a jump in $V_{x}$ across $x_{0}^{i}$. These eigenvalues will be called "singular" critical eigenvalues. There may be "regular" critical eigenvalues that satisfy the reduced eigenvalue problem (1.12) with $V \in C^{1}[0,1]$. Calculation of regular critical eigenvalues is quite different from the procedure given in this paper. From (H1) there exists $\gamma_{1}>0$ such that if $\operatorname{Re} \lambda>-\gamma_{1}$ then $\left(f_{u}-\lambda\right)^{-1}$ exists. To avoid regular critical eigenvalues, we assume that

(H9) There exists $\gamma>0$ such that for $\operatorname{Re} \lambda>-\gamma$, the following equation

$$
V_{x x}-\left(\lambda+g_{u}\left(f_{u}-\lambda\right)^{-1} f_{v}-g_{v}\right) V=0,
$$

with boundary conditions (4.11) does not have any piecewise smooth, nonzero solution that is in $C^{1}[0,1]$.

Notice that (7.1) comes from (1.12) by setting $\epsilon=0$. With (H9), it is easy to show that there is no regular critical eigenvalue in the region $\operatorname{Re} \lambda>-\gamma$.

For system (6.4) with $k=0$, Nishiura and Fujii showed that (H9) is satisfied 39]. If $k / \omega$ is sufficiently small, (H9) can be verified easily as a small perturbation to the one with $k=0$.

3. To use the expansions of critical eigenvalues in the stability analysis, we need to prove that, in the region $\operatorname{Re} \lambda \geq-\beta_{0}>-\gamma$, all the eigenvalues are the singular critical eigenvalues obtained in $\S 4$.

Consider an asymptotic series $\mu(\epsilon)=\sum_{j} \epsilon^{j} \mu_{j}$ with $\mu_{0}>-\beta_{0}$. Assume that $\mu(\epsilon)$ is not equal to any of the critical eigenvalues obtained in this paper. That is, for any critical eigenvalue $\lambda^{i}(\epsilon), 1 \leq i \leq r$, there exists an integer $j_{0}^{i}$ such that $\mu_{j}=\lambda_{j}^{i}, j<j_{0}^{i}$ but $\mu_{j 0} \neq \lambda_{j 0}^{i}$. Let

$$
j_{0}=\max \left\{j_{0}^{i}, 1 \leq i \leq r\right\} .
$$

We want to show that $\mu(\epsilon)$ is a regular value. Note that if the corresponding eigenfunction of $\mu(\epsilon)$ has an asymptotic expansion in $\epsilon$, then we know that $\mu(\epsilon)$ is not an singular critical eigenvalue due to results of the previous sections. However, since we can not assume that the corresponding eigenfunction has an asymptotic expansion in $\epsilon$, the result needs to be proved separately.

Assume that $h(x, \epsilon)=\left(h^{u}(x, \epsilon), h^{v}(x, \epsilon)\right)$ is $C^{\infty}$ and admits asymptotic expansions in the same regular and singular layers defined by the internal layer solution $(u(x, \epsilon), v(x, \epsilon))$. Consider the resolvent problem

$$
\begin{aligned}
& \mu U=\epsilon^{2} U_{x x}+f_{u} U+f_{v} V+h^{u}, \\
& \mu V=V_{x x}+g_{u} U+g_{v} V+h^{v},
\end{aligned}
$$

with suitable boundary conditions at $x=0,1$. We look for a matched formal series solution $(U(\epsilon), V(\epsilon))$. Denote the above as

$$
\mu \Xi-\mathfrak{A}(\epsilon) \Xi=h(\epsilon), \quad \Xi=(U, V), \quad h=\left(h^{u}, h^{v}\right) .
$$

We show formally that the inverse of $\mu-\mathfrak{A}$ exists, with $(\mu-\mathfrak{A})^{-1}=O\left(\epsilon^{-j_{0}}\right)$. 
Theorem 7.2. Assume that $\mu(\epsilon)=\sum_{j} \epsilon^{j} \mu_{j}, \mu_{0}>-\beta_{0}=\min \left\{\gamma_{0}, \gamma\right\}$, is an asymptotic series that is not equal to any of the critical eigenvalues obtained in this paper. Let $j_{0}$ be the largest of the powers as above. Then for any $h(\epsilon)=\sum_{j} \epsilon^{j} h^{j}$ with $h_{j}=0$ for $j<j_{0}$ and $h_{j_{0}} \neq 0$, the eigenvalue problem (7.2) has a unique matched formal series solution $\Xi=\sum_{j=0}^{\infty} \epsilon^{j} \Xi_{j}$.

Proof. Case 1. $j_{0}=0$, i.e. $\mu_{0} \neq 0$.

Consider the $\epsilon^{0}$ th expansion:

In regular layers,

$$
\begin{aligned}
\mu_{0} U_{0}^{R} & =f_{u} U_{0}^{R}+f_{v} V_{0}^{R}+h_{0}^{u}, \\
U_{0}^{R} & =\left(\mu_{0}-f_{u}\right)^{-1}\left(f_{v} V_{0}^{R}+h_{0}^{u}\right), \\
\mu_{0} V_{0}^{R} & =V_{0 x x}^{R}+\left[g_{u}\left(\mu_{0}-f_{u}\right)^{-1} f_{v}+g_{v}\right] V_{0}^{R}+g_{u}\left(\mu_{0}-f_{u}\right)^{-1} h_{0}^{u}+h_{0}^{v} .
\end{aligned}
$$

From (H1) and (H9), if we know the jumps $\left(V_{0}^{R}, V_{0 x}^{R}\right)$ at $x_{0}^{i}$, we can solve for $\left(U_{0}^{R}, V_{0}^{R}\right)$.

In the $i$ th internal layer,

$$
V_{0 \xi}^{S}=0, W_{0 \xi}^{S}=0, \quad V_{0}^{S}=\text { constant, } W_{0}^{S}=\text { constant. }
$$

It means that there is no jump for $\left(V_{0}^{R}, W_{0}^{R}\right)$ across $x_{0}^{i}$. Thus we can solve for $V_{0}^{R}$. We also have $V_{0}^{S}=V_{0}^{R}\left(x_{0}^{i}\right)$.

$$
\mu_{0} U_{0}^{S}=U_{0 \xi \xi}^{S}+f_{u} U_{0}^{S}+f_{v} V_{0}^{S}+h_{0}^{u} .
$$

From (H2), $\mu_{0}$ is not an eigenvalue for the above. One can uniquely solve for $U_{0}^{S}$.

In the $\epsilon^{j}$ th expansion, we can solve for $\left(U_{j}, V_{j}\right)$ both in regular and singular layers much like the same way for $\left(U_{0}, V_{0}\right)$.

Case 2. $j_{0}=1$. In this case $\mu_{0}=0, \mu_{1} \neq \lambda_{1}^{i}$ for any $1 \leq i \leq r$ and $h_{0}=0$ but $h_{1} \neq 0$.

In the $\epsilon^{0}$ th expansion, since $\lambda_{0}=0, h_{0}=0$, we have

$$
\begin{array}{r}
U_{0}^{R}=0, V_{0}^{R}=0, \quad \text { in regular layers, } \\
V_{0}^{S i}=0, U_{0}^{S i}=d_{0}^{i} \dot{q}^{i}, \quad \text { in the } i \text { th singular layer. }
\end{array}
$$

$d_{0}^{i}$ remains to be determined.

Consider the $\epsilon^{1}$ th expansion:

In the regular layer, since $\mu_{0}=0, U_{0}^{R}=0, V_{0}^{R}=0$, then

$$
\begin{aligned}
0 & =f_{u} U_{1}^{R}+f_{v} V_{1}^{R}+h_{1}^{u}, \\
U_{1}^{R} & =-f_{u}^{-1}\left(f_{v} V_{1}^{R}+h_{1}^{u}\right), \\
V_{1 x x}^{R} & -\left(g_{u} f_{u}^{-1} f_{v}-g_{v}\right) V_{1}^{R}=g_{u} f_{u}^{-1} h_{1}^{u}-h_{1}^{v} .
\end{aligned}
$$

From (H1) and Lemma 4.1, if we know the jump of $\left(V_{1}^{R}, V_{1 x}^{R}\right)$ across each $x_{0}^{i}$, we can solve for $\left(U_{1}^{R}, V_{1}^{R}\right)$.

In the $i$ th singular layer, since $V_{0}^{S}=0, \mu_{0} V_{1}^{S}+\mu_{1} V_{0}^{S}=0$, then

$$
\begin{gathered}
V_{1 \xi}^{S}=W_{0}^{S}=0, \\
W_{1 \xi}^{S}=-g_{u} d_{0}^{i} \dot{q}^{i} .
\end{gathered}
$$


Integrate from $\xi=-\infty$ to $\infty$, and use the matching principle,

$$
\begin{aligned}
V_{1}^{R}\left(x_{0}^{i}+\right)-V_{1}^{R}\left(x_{0}^{i}-\right) & =0, \\
W_{1}^{R}\left(x_{0}^{i}+\right)-W_{1}^{R}\left(x_{0}^{i}-\right) & =d_{0}^{i} \mathcal{M}^{i} .
\end{aligned}
$$

We can now solve for $\left(V_{1}^{R}, W_{1}^{R}\right)$. They are of the form

$$
V_{1}^{R}=\sum_{1}^{r} d_{0}^{i} V_{c}^{i}+\ell \cdot o \cdot t, \quad W_{1}^{R}=\sum_{1}^{r} d_{0}^{i} W_{c}^{i}+\ell \cdot o \cdot t
$$

Here $\ell \cdot o \cdot t$ involves $h_{1}$. We have $V_{1}^{S}=V_{1}^{R}\left(x_{0}^{i}\right)$. Plugging into

$$
\mu_{1} d_{0}^{i} \dot{q}^{i}=U_{1 \xi \xi}^{S}+f_{u} U_{1}^{S}+f_{v} V_{1}^{S}+d_{0}^{i}\left(f_{u u} \dot{q}^{i} u_{1}+f_{u v} \dot{q}^{i} v_{1}\right)+h_{1}^{u},
$$

we can solve for $U_{1}^{S}$ if the nonhomogeneous terms are orthogonal to $\psi^{i}$. Integrating by parts as in (4.19),

$$
\begin{array}{r}
d_{0}^{i} \mu_{1}=\sum_{\ell=1}^{r} d_{0}^{\ell} \mathbf{n}^{i} \cdot V_{c}^{\ell}\left(x_{0}^{i}\right)-d_{0}^{i} \mathbf{n}^{i} \cdot w_{0}^{R}\left(x_{0}^{i}\right)+\left\langle\psi^{i}, h_{1}^{u}\right\rangle+\ell \cdot o \cdot t . \\
\mu_{1}\left(\begin{array}{c}
d_{0}^{1} \\
\vdots \\
d_{0}^{r}
\end{array}\right)=A\left(\begin{array}{c}
d_{0}^{1} \\
\vdots \\
d_{0}^{r}
\end{array}\right)+\left(\begin{array}{c}
\left\langle\psi^{1}, h_{1}^{u 1}\right\rangle \\
\vdots \\
\left\langle\psi^{r}, h_{1}^{u r}\right\rangle
\end{array}\right)+\ell \cdot o \cdot t .
\end{array}
$$

Since $\mu_{1} \neq \lambda_{1}^{i}, 1 \leq i \leq r, \mu_{1}$ is not an eigenvalue. The above has a unique solution $\left(d_{0}^{1}, \cdots, d_{0}^{r}\right)$.

Assume that the $\epsilon^{j-1}$ th expansion has been obtained. We have $U_{j-1}^{S i}=d_{j-1}^{i} \dot{q}^{i}+$ $Z_{j-1}^{i}$ where $Z_{j-1}^{i}$ has uniquely been determined but $d_{j-1}^{i}$ has not. In the $\epsilon^{j}$ th expansion, we can similarly show that

$$
\begin{aligned}
& d_{j-1}^{i} \mu_{1}=\sum_{\ell=1}^{r} d_{j-1}^{\ell} \mathbf{n}^{i} \cdot V_{c}^{\ell}\left(x_{0}^{i}\right)-d_{j-1}^{i} \mathbf{n}^{i} \cdot w_{0}^{R}\left(x_{0}^{i}\right)+\left\langle\psi^{i}, h_{j}^{u}\right\rangle+\ell \cdot o \cdot t . \\
& \mu_{1}\left(\begin{array}{c}
d_{j-1}^{1} \\
\vdots \\
d_{j-1}^{r}
\end{array}\right)=A\left(\begin{array}{c}
d_{j-1}^{1} \\
\vdots \\
d_{j-1}^{r}
\end{array}\right)+\left(\begin{array}{c}
\left\langle\psi^{1}, h_{j}^{u 1}\right\rangle \\
\vdots \\
\left\langle\psi^{r}, h_{j}^{u r}\right\rangle
\end{array}\right)+\ell \cdot o \cdot t .
\end{aligned}
$$

From this, we can solve for $\left(d_{j-1}^{1}, \cdots, d_{j-1}^{r}\right)$.

Case 3. $j_{0}>1$. In this case there exists an critical eigenvalue $\lambda^{i}(\epsilon)$ such that $\mu_{j}=\lambda_{j}^{i}, j<j_{0}, \mu_{j_{0}} \neq \lambda_{j_{0}}^{i}$. We shall solve

$$
\mu \Xi-\mathfrak{A}(\epsilon) \Xi=h(\epsilon)
$$

where $h_{j}=0$ for $j<j_{0}, h_{j_{0}} \neq 0$.

An important observation is that it suffices to find asymptotic series for (7.3) up to the $\epsilon^{j_{0}}$ th expansion. Let the normalized eigenfunction corresponding to $\lambda^{i}(\epsilon)$ be

$$
\left(\bar{U}^{i}(\epsilon), \bar{V}^{i}(\epsilon)\right)=\left(\sum \epsilon^{j} \bar{U}_{j}^{i}, \sum \epsilon^{j} \bar{V}_{j}^{i}\right) .
$$

In the future, we drop the index $i$ on $\lambda_{j}^{i}$ and $\left(\bar{U}^{i}, \bar{V}^{i}\right)$. For expansions to the order $\epsilon^{j}, j<j_{0}$, since $h_{j}=0$, and $\mu_{j}=\lambda_{j}$, we have the same equations as the eigenvalue-eigenfunction equations. Therefore, we set

$$
U_{j}=k_{0} \bar{U}_{j}, \quad V_{j}=k_{0} \bar{V}_{j}, \quad W_{j}=k_{0} \bar{W}_{j}, \quad j<j_{0},
$$


except for $U_{j_{0}-1}^{S}=k_{0} \bar{U}_{j_{0}-1}^{S}+d^{i} \dot{q}^{i}$ in the $i$ th internal layer. The parameters $\left(d^{1}, \cdots, d^{r}\right)$ remain to be determined.

Consider the $\epsilon^{j_{0}}$ th expansion. Let

$$
\begin{aligned}
U_{j_{0}} & =k_{0} \bar{U}_{j_{0}}+U, \\
V_{j_{0}} & =k_{0} \bar{V}_{j_{0}}+V, \\
W_{j_{0}} & =k_{0} \bar{W}_{j_{0}}+W .
\end{aligned}
$$

Here $U=U^{S}, V=V^{S}, W=W^{S}$ in internal layers, and $U=U^{R}, V=V^{R}, W=$ $W^{R}$ in regular layers.

In regular layers, since $(\bar{U}(\epsilon), \bar{V}(\epsilon))$ satisfies the eigenvalue equations, then all the terms multiplied by $k_{0}$ should cancel. In the $\epsilon^{j_{0}}$ th expansion, after the cancellation, we have

$$
\begin{aligned}
0 & =f_{u} U^{R}+f_{v} V^{R}+h_{j_{0}}^{u}, \\
0 & =V_{x x}^{R}+g_{u} U^{R}+g_{v} V^{R}+h_{j_{0}}^{v}, \\
U^{R} & =-f_{u}^{-1} f_{v} V^{R}-f_{u}^{-1} h_{j_{0}}^{u}, \\
0 & =V_{x x}^{R}-g_{u}\left(f_{u}^{-1} f_{v} V^{R}-f_{u}^{-1} h_{j_{0}}^{u}\right)+g_{v} V^{R}+h_{j_{0}}^{v} .
\end{aligned}
$$

We can solve for $(U, V)$ if jumps of $\left(V^{R}, V_{x}^{R}\right)$ across $x_{0}^{i}$ are obtained.

In the $i$-th internal layer, we again can cancel all the terms in both sides of the equation that are also in the eigenvalue equation. Since

$$
\mu_{o} U_{j_{0}}^{S}+\cdots+\mu_{j_{0}} U_{0}^{S}=k_{0}\left(\lambda_{1} \bar{U}_{j_{0}-1}^{S}+\cdots+\lambda_{j_{0}} \bar{U}_{0}^{S}\right)+\lambda_{1} d^{i} \dot{q}^{i}+k_{0}\left(\mu_{j_{0}}-\lambda_{j_{0}}\right) \bar{U}_{0}^{S} .
$$

After the cancellation,

$$
\begin{gathered}
\lambda_{1} d^{i} \dot{q}^{i}+k_{0}\left(\mu_{j_{0}}-\lambda_{j_{0}}\right) \bar{U}_{0}^{S}=U_{\xi \xi}^{S}+f_{u} U^{S}+f_{v} V^{S}+h_{j_{0}}^{u} . \\
V_{\xi}^{S}=0, \\
W_{\xi}^{S}=-d^{i} g_{u} \dot{q}^{i}, \\
V^{R}\left(x_{0}^{i}+\right)-V^{R}\left(x_{0}^{i}-\right)=0, \\
W^{R}\left(x_{0}^{i}+\right)-W^{R}\left(x_{0}^{i}-\right)=d^{i} \mathcal{M}^{i} .
\end{gathered}
$$

The solution of $V^{R}$ has the form

$$
V^{R}=\sum_{\ell=1}^{r} d^{\ell} V_{c}^{\ell}+O\left(\left|h_{j_{0}}\right|\right) .
$$

Substituting $V^{S}=V^{R}\left(x_{0}^{i}\right)$ into the equation for $U^{S}$, using the Fredholm alternative, and integrating by parts as (4.19), we have

$$
\begin{gathered}
\lambda_{1} d^{i}+k_{0}\left(\mu_{j_{0}}-\lambda_{j_{0}}\right) c_{0}^{i}=\mathbf{n}^{i} \cdot\left[\sum_{\ell=1}^{r} d^{\ell} V_{c}^{\ell}\left(x_{0}^{i}\right)-d^{i} w_{0}^{R}\left(x_{0}^{i}\right)\right]+O\left(\left|h_{j_{0}}\right|\right) . \\
\lambda_{1}\left(\begin{array}{c}
d^{1} \\
\vdots \\
d^{r}
\end{array}\right)=A\left(\begin{array}{c}
d^{1} \\
\vdots \\
d^{r}
\end{array}\right)-k_{0}\left(\mu_{j_{0}}-\lambda_{j_{0}}\right)\left(\begin{array}{c}
c_{0}^{1} \\
\vdots \\
c_{0}^{r}
\end{array}\right)+O\left(\left|h_{j_{0}}\right|\right) .
\end{gathered}
$$

Since $\left(c_{0}^{1}, \ldots, c_{0}^{r}\right)^{\tau}$ is in the kernel of $\left(\lambda_{1}-A\right)$, it is not in the range of $\lambda_{1} I-A$. Since $\mu_{j_{0}} \neq \lambda_{j_{0}}$, there exists a unique $k_{0}$ that allows the equation for $\left(d^{1}, \ldots, d^{r}\right)$ to be solved. Without loss of generality, let $\left(d^{1}, \ldots, d^{r}\right) \perp\left(c_{0}^{1}, \ldots, c_{0}^{r}\right)$. 
After the $\epsilon^{j_{0}}$ th order expansion has been obtained, we can compute the $\epsilon^{j_{0}+1}$ th and other higher order expansions by induction, with the similar method.

The series expansion is a formal solution to the resolvent problem. With the help of some contraction mappings and iteration methods, similar to the ones outlined in the Appendix, one can show that there exists a small $\epsilon_{0}>0$ such that if $\epsilon \leq \epsilon_{0}$, then $\sum_{0}^{j_{0}} \epsilon^{j} \mu_{j}$ is a regular value of the internal layer solution. The constant $\epsilon_{0}$ depends on $\left|\mu_{j_{0}}-\lambda_{j_{0}}\right|$.

Denote the critical eigenvalues by $\lambda^{(\ell)}(\epsilon)=\sum_{0}^{\infty} \epsilon^{j} \lambda_{j}^{(\ell)}, 1 \leq \ell \leq r$. A critical eigenvalue $\lambda^{(\ell)}(\epsilon)$ is said to be stable if $\operatorname{Re} \lambda_{1}^{(\ell)}<0$. It is said to be unstable if $\operatorname{Re} \lambda_{j}^{(\ell)}>0$. We can show the following

Theorem 7.3. With (H9), there exists a constant $\epsilon_{0}>0$ such that if $0<\epsilon \leq \epsilon_{0}$, then the internal layer solution is unstable if there exists at least one unstable critical eigenvalue; the internal layer solution is stable if all the critical eigenvalues are stable.

Proof. Only the idea of the proof is given. First if $\lambda^{(\ell)}(\epsilon)$ is an unstable eigenvalue with $\operatorname{Re} \lambda_{1}^{\ell}>0$, then from Theorem A.4 there exists a true eigenvalue of the internal layer solution in the right half complex plane if $\epsilon$ is sufficiently small. Thus the internal layer solution is unstable.

Next assume that all the critical eigenvalues are stable. There exist $\epsilon_{0}>0$ such that all the truncated eigenvalues $\lambda^{\ell}(\epsilon)=\epsilon \lambda_{1}^{(\ell)}$ lie in the left half complex plane provided that $0<\epsilon<\epsilon_{0}$. A cone centered at $\lambda^{\ell}(\epsilon)$ is defined as $\left\{\epsilon \lambda:\left|\lambda-\lambda_{1}^{\ell}\right| \leq \delta\right\}$ and is called an $\ell-\delta$ cone. We choose $\delta>0$ so that all such cones lie in the left half complex plane for $0<\epsilon \leq \epsilon_{0}$.

Let $\mu$ be a complex number with $\operatorname{Re} \mu \geq 0$. Then for some sufficiently small $\delta$, $\mu$ is not in any of the $\ell-\delta$ cones. From Theorem 7.2 formally $\mu$ is a regular value. Using a contraction mapping argument, we can show rigorously that there exists a small $\epsilon_{0}$ such that $\mu$ is a regular value if $0<\epsilon<\epsilon_{0}$. Care must be taken to ensure that a common $\epsilon_{0}$ can be found for all such $\mu$. Details will be omitted due to the length of the paper. We have shown that all the eigenvalues are in the left plane $\operatorname{Re} \lambda<0$, therefore the internal layer solution is stable.

\section{Appendix A. The existence of the LAYer solutions And the CRitical EIGENVALUE-EIGENFUNCTIONS}

The iteration method as stated in Lemma A.1 will be used throughout this section. Let $\mathcal{L}$ be a bounded linear operator from Banach spaces $E_{1}$ to $E_{2}$. We say $\mathcal{S}: E_{2} \rightarrow E_{1}$ is an approximate right inverse of $\mathcal{L}$ if $|I-\mathcal{L S}|<1$.

Lemma A.1. If $\mathcal{L}$ has an approximate right inverse $\mathcal{S}$, then the abstract equation $\mathcal{L} x=y$ has a (nonunique) solution $x=\mathcal{S} \sum_{0}^{\infty}(I-\mathcal{L S})^{j} y$. If moreover, $\mathcal{S}$ is invertible, then the solution is unique.

In practice, $E_{1}$ is the space of solutions and $E_{2}$ is the space of forcing functions plus the space of boundary and jump terms related to a system of differential equations. The operator $\mathcal{S}$ is usually the inverse of a simplified operator $\mathcal{L}_{1}$ derived from $\mathcal{L}$ by dropping some coefficients, changing the forcing terms, jump terms or deforming the domain of solutions. If $\mathcal{S}=\mathcal{L}_{1}^{-1}, \mathcal{L}_{1} x_{1}=y$, then $|I-\mathcal{L S}|=C_{1}<1$ 
means

$$
\left|\left(\mathcal{L}_{1}-\mathcal{L}\right)\left(x_{1}\right)\right|<C|y|, \quad \text { for all } x_{1} \in E_{1} .
$$

Condition A.1 can be checked, a posteriori, without using the exact solution $x=\mathcal{L}^{-1} y$. To solve a difficult abstract equation, we may need to find a finite chain of operators: $\mathcal{L}_{j}, 1 \leq j \leq k$, satisfying (A.1) for any two adjacent operators. The last equation $\mathcal{L}_{k} x_{1}=y$ must be easy to solve.

For any integer $m \geq 0$, let $x_{a p}^{\ell}(\epsilon)=\sum_{0}^{m} \epsilon^{j} x_{j}^{\ell}, 1 \leq \ell \leq r$, be an approximation of the position of the $\ell$ th internal layer. We look for $\Delta x^{\ell}$ so that $x_{\Delta}^{\ell}=x_{a p}^{\ell}(\epsilon)+\Delta x^{\ell}$ is the exact layer position. For convenience, we set $\Delta x^{\ell}=0$ for $\ell=0, r+1$ and let $x_{\Delta}^{0}=x_{a p}^{0}=0$ and $x_{\Delta}^{r+1}=x_{a p}^{r+1}=1$. Let $\epsilon^{\beta}, 0<\beta<1$, be an "intermediate variable". Define a sequence of points $a^{i}, 0 \leq i \leq 2 r+3$. Except for $a^{0}=0$ and $a^{2 r+3}=1$, points $a^{1}$ to $a^{2 r+2}$ are defined as

$$
\begin{aligned}
a^{2 i} & =x_{\Delta}^{i}-\epsilon^{\beta}, & & 1 \leq i \leq r+1, \\
a^{2 i+1} & =x_{\Delta}^{i}+\epsilon^{\beta}, & & 0 \leq i \leq r .
\end{aligned}
$$

The interval $[0,1]$ is divided by $\left\{a^{i}\right\}_{i=0}^{2 r+3}$ into $2 r+3$ subintervals that alternatively house singular and regular layers, see Figure A.1.

$$
\begin{aligned}
& I^{i}=\left\{x \mid a^{i-1}<x<a^{i}, 1 \leq i \leq 2 r+3\right\}, \\
& I^{2 \ell+1}, 0 \leq \ell \leq r+1, \text { are for the }(r+2) \text {-singular layers, } \\
& I^{2 \ell}, 1 \leq \ell \leq r+1, \text { are for the }(r+1) \text {-regular layers. }
\end{aligned}
$$

Let $\xi^{i}=a^{i} / \epsilon$. Then in the stretched variable $\xi=x / \epsilon, I^{i}=\left\{\xi \mid \xi^{i-1}<\xi<\xi^{i}\right\}$. The width of a singular layer is $O\left(\epsilon^{\beta}\right)$ in the $x$-variable, but is $O\left(\epsilon^{\beta-1}\right)>>1$ in the $\xi$ variable. The interval $I^{i}$ is also called regular or singular layers if $i=2 \ell$ or $2 \ell+1$. If $\Delta x^{\ell}=0$ for $\ell=1, \ldots, r$, then the corresponding unperturbed sequences of points and intervals are denoted by $a_{0}^{i}, \xi_{0}^{i}$ and $I_{0}^{i}$.

For any integer $m \geq 0$, define the $\epsilon^{m}$ th approximations of eigenvalues, internal layer solutions and eigenfunctions with $W=(u, v)$ and $W=(U, V)$ respectively, by truncating the asymptotic series as follows:

$$
\begin{array}{rlrl}
\lambda_{a p}^{k} & =\sum_{0}^{m} \epsilon^{j} \lambda_{j}^{k}, & k & =1, \ldots, r, \\
W_{a p}(x, \epsilon) & =\sum_{0}^{m} \epsilon^{j} W_{j}^{R}(x), & x \in I^{i}, i=2 \ell, 1 \leq \ell \leq r+1, \\
W_{a p}(x, \epsilon) & =\sum_{0}^{m} \epsilon^{j} W_{j}^{S \ell}\left(\left(x-x_{\Delta}^{\ell}\right) / \epsilon, \epsilon\right), & & x \in I^{i}, i=2 \ell+1,0 \leq \ell \leq r+1 .
\end{array}
$$

A function $W$ in $I^{i}$ will be denoted $W^{i}$ if necessary.

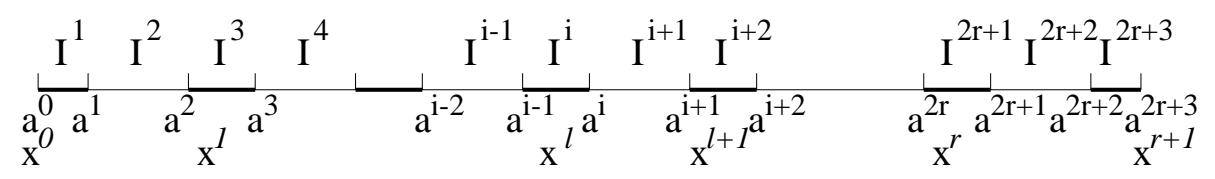

Figure A.1. The partition of singular and regular layers where $i=2 \ell+1, x^{\ell} \in I^{i}=I^{2 \ell+1}$, the $\ell$ th internal layer. 
Although the interval $I^{i}$ varies with $\Delta x^{i}$ and $\epsilon, W_{a p}$ is still well defined. In regular layers, using the differential equations, the domain of $W_{j}^{R}(x)$ can be extended from $x \in R^{\ell}=\left(x_{0}^{\ell-1}, x_{0}^{\ell}\right)$ to an open interval $O^{i}$ containing $R^{\ell}$. Therefore, if $\epsilon$ and $\max _{\ell}\left\{\Delta x^{\ell}\right\}$ are sufficiently small, then $I^{i} \subset O^{i}$ so that $W_{a p}(x, \epsilon)$ is defined in $I^{i}$. In a singular layer, $W_{a p}(x, \epsilon)$ is only shifted in the $x$ direction when $\Delta x^{\ell} \neq 0$.

Formal approximation of internal layer solutions. $\left(u_{a p}, v_{a p}\right)$ defined above is a formal approximation in the sense that after substituting into (4.1), the residual errors in all layers, boundary errors at $x=0,1$, and jump errors between adjacent singular and regular layers are small.

If we let $\left(-F^{i},-G^{i}\right)$ be the residual error of the approximation in $I^{i}$, then

$$
\begin{aligned}
& u_{a p, \xi \xi}+f\left(u_{a p}, v_{a p}\right)=-F^{i}, \\
& v_{a p, x x}+g\left(u_{a p}, v_{a p}\right)=-G^{i}, \quad 1 \leq i \leq 2 r+3 .
\end{aligned}
$$

All the norms in this section are supremum norms unless otherwise specified. It is easy to verify that $\left|F^{i}\right|+\left|G^{i}\right|=O\left(\epsilon^{m+1}\right)$ in regular layers. In singular layers, the Taylor expansion of $f$ and $g$ involves polynomial growth terms of $\xi$. Since the layer width in $\xi$ is of $\epsilon^{\beta-1}$, the residual error due to truncation of $f$ is $O\left(\epsilon^{m+1} \xi^{m+1}\right)=$ $O\left(\epsilon^{\beta(m+1)}\right.$. In singular layers, only the $\epsilon^{m-1}$ th order expansion of $g$ was used due to the extra term $\epsilon$ in front of $g$, thus the truncation error $\left|G^{i}\right|=O\left(\epsilon^{m \beta}\right)$. In the $x$ scale, the $L^{1}$ norm is $O\left(\epsilon^{\beta(m+1)}\right)$. In conclusion

$$
\begin{aligned}
\left|F^{i}\right|+\left|G^{i}\right| & =O\left(\epsilon^{m+1}\right), \quad \text { in regular layers, } \\
\left|F^{i}\right|+\left|G^{i}\right|_{L^{1}} & =O\left(\epsilon^{\beta(m+1)}\right), \quad \text { in singular layers. }
\end{aligned}
$$

If we define the jump errors between layers with $\Delta x^{\ell}=0, \ell=1, \ldots, r$, as

$$
\begin{array}{cc}
u_{a p}^{i+1}\left(a_{0}^{i}\right)-u_{a p}^{i}\left(a_{0}^{i}\right)=-J_{1}^{i}, & u_{a p, x}^{i+1}\left(a_{0}^{i}\right)-u_{a p, x}^{i}\left(a_{0}^{i}\right)=-J_{2}^{i}, \\
v_{a p}^{i+1}\left(a_{0}^{i}\right)-v_{a p}^{i}\left(a_{0}^{i}\right)=-J_{3}^{i}, & v_{a p, x}^{i+1}\left(a_{0}^{i}\right)-v_{a p, x}^{i}\left(a_{0}^{i}\right)=-J_{4}^{i},
\end{array}
$$

then we have

$$
\sum_{i=1}^{4} \sum_{j=1}^{4}\left|J_{j}^{i}\right| \leq C \epsilon^{\beta(m+1)} .
$$

For a proof see $[29,30,18$.

Existence of internal layer solutions. Let $\left(u_{a p}+u, v_{a p}+v\right)$ be the exact solution with the exact layer position $x_{a p}^{\ell}(\epsilon)+\Delta x^{\ell}$. The functions $(u, v)$ satisfy the following linear variational equations. In regular layers,

$$
\begin{aligned}
& u_{\xi \xi}+f_{u}^{i} u+f_{v}^{i} v=F^{i}(\xi)+M^{i}(u, v, \epsilon), \\
& v_{x x}+g_{u}^{i} u+g_{v}^{i} v=G^{i}(x)+N^{i}(u, v, \epsilon) .
\end{aligned}
$$

In singular layers,

$$
\begin{aligned}
& u_{\xi \xi}+f_{u}^{i} u+f_{v}^{i} v=F^{i}(\xi)+M^{i}(u, v, \epsilon), \\
& v_{x x}=G^{i}(x)+N^{i}(u, v, \epsilon) .
\end{aligned}
$$

The coefficients are based on linearizing at the $\epsilon^{0}$ th order approximations. For example, in regular layers, $f_{u}^{i}=f_{u}\left(u_{0}^{R \ell}(x), v_{0}^{R \ell}(x)\right), i=2 \ell$. In singular layers, $f_{u}^{i}=f_{u}^{i}\left(u_{0}^{S \ell}\left(\xi-x_{0}^{\ell} / \epsilon\right), v_{0}^{S \ell}\left(\xi-x_{0}^{\ell} / \epsilon\right)\right), i=2 \ell+1$. Similar definitions apply to 
$f_{v}^{i}, g_{u}^{i}, g_{v}^{i}$. If $\xi$ is used in regular layers, let $x=\epsilon \xi$ and if $x$ is used in singular layers, let $\xi=x / \epsilon$.

A direct linearization would yield $v_{x x}+g_{u}^{i} u+g_{v}^{i} v=G^{i}(x)+N^{i}(u, v, \epsilon)$ in singular layers. But since the length of the layer is $O\left(\epsilon^{\beta}\right), g_{u}^{i} u+g_{v}^{i} v=O\left(\epsilon^{\beta}(|u|+|v|)\right)$ and is included in $N^{i}$.

The nonlinear terms satisfy,

$$
\left|M^{i}\right|+\left|N^{i}\right|_{L^{1}} \leq C\left(\left|u^{i}\right|^{2}+\left|v^{i}\right|^{2}+\epsilon^{\beta}(|u|+|v|)\right) .
$$

Let $I^{i}, i=2 \ell+1$, be a singular layer. Observe that adding $\Delta x^{\ell}$ does not change the values of $u^{i}$ at the boundaries of $I^{i}$. When $\Delta x^{\ell} \neq 0$ the jump conditions for $(u, v)$ are

$$
\begin{aligned}
& u^{i+1}\left(a^{i}\right)-u^{i}\left(a^{i}\right)=u_{a p}^{i}\left(a^{i}\right)-u_{a p}^{i+1}\left(a^{i}\right), \\
& u^{i}\left(a^{i-1}\right)-u^{i-1}\left(a^{i-1}\right)=u_{a p}^{i-1}\left(a^{i-1}\right)-u_{a p}^{i}\left(a^{i-1}\right) .
\end{aligned}
$$

After linearization, we have

$$
\begin{aligned}
& u^{i+1}\left(a^{i}\right)-u^{i}\left(a^{i}\right)=J_{1}^{i}-u_{0 x}^{R, \ell+1}\left(x_{0}^{\ell}+\right) \Delta x^{\ell}+K_{1}^{i}, \\
& u^{i}\left(a^{i-1}\right)-u^{i-1}\left(a^{i-1}\right)=J_{1}^{i-1}+u_{0 x}^{R, \ell}\left(x_{0}^{\ell}-\right) \Delta x^{\ell}+K_{1}^{i-1} .
\end{aligned}
$$

The nonlinear terms satisfy

$$
\begin{aligned}
K_{1}^{i} & =O\left(\left|\Delta x^{\ell}\right|^{2}+\left|u^{i+1}\right|^{2}+\epsilon^{\beta}\left(\left|\Delta x^{\ell}\right|+\left|u^{i+1}\right|\right),\right. \\
K_{1}^{i-1} & =O\left(\left|\Delta x^{\ell}\right|^{2}+\left|u^{i-1}\right|^{2}+\epsilon^{\beta}\left(\left|\Delta x^{\ell}\right|+\left|u^{i-1}\right|\right) .\right.
\end{aligned}
$$

Similar formulas for the jumps of $u_{x}, v, v_{x}$ can be written at the junction points.

If we can solve the following system of linear nonhomogeneous equations, then the nonlinear system can be solved by the contraction mapping principle.

In a regular layer $I^{i}, i=2 \ell, 1 \leq \ell \leq r+1$,

$$
\begin{aligned}
& u_{\xi \xi}+f_{u}^{i} u+f_{v}^{i} v=F^{i}(\xi), \\
& v_{x x}+g_{u}^{i} u+g_{v}^{i} v=G^{i}(x) .
\end{aligned}
$$

In a singular layer $I^{i}, i=2 \ell+1,1 \leq \ell \leq r+1$,

$$
\begin{aligned}
u_{\xi \xi}+f_{u}^{i} u+f_{v}^{i} v & =F^{i}(\xi), \\
v_{x x} & =G^{i}(x) .
\end{aligned}
$$

The boundary conditions at $x=0,1$ are

$$
\begin{aligned}
& u_{x}(0)=u_{x}(1)=0, \\
& A_{j} v_{x}(j)+B_{j} v(j)=0, \quad j=0,1 .
\end{aligned}
$$

Denote $u, u_{x}, v, v_{x}$ by $z$, the jump conditions for $i=2 \ell+1, I^{i}$ a singular layer, are

$$
\begin{aligned}
z^{i+1}\left(a^{i}\right)-z^{i}\left(a^{i}\right) & =J_{j}^{i}-z_{0 x}^{R}\left(x_{0}^{\ell}+\right) \Delta x^{\ell}, \\
z^{i}\left(a^{i-1}\right)-z^{i-1}\left(a^{i-1}\right) & =J_{j}^{i-1}+z_{0 x}^{R}\left(x_{0}^{\ell}-\right) \Delta x^{\ell},
\end{aligned}
$$

where $j=1,2,3,4$ if $z=u, u_{x}, v, v_{x}$ respectively.

We can prove the following result 
Theorem A.2. The system (A.4)-(A.7) with boundary conditions (A.8) and jump conditions (A.9) has a unique solution $\left(u, v,\left\{\Delta x^{i}\right\}_{1}^{r}\right)$ that satisfies

$$
\left|\left\{\Delta x^{i}\right\}\right|+|u|+|v| \leq C\left\{\sum\left|F^{i}\right|+\sum\left|G^{i}\right|_{L^{1}}+\sum_{i} \sum_{j=1}^{4}\left|J_{j}^{i}\right|\right\} .
$$

By the superposition principle, the proof is divided into two steps.

(1) STEP ONE: We solve the nonhomogeneous system (A.4)-(A.7) in each layer, taking care of the boundary conditions (A.8) but ignoring the jump conditions (A.9).

(2) STEP TWO: We solve a homogeneous system (A.4)-(A.7) with zero $\left(F^{i}, G^{i}\right)$ and zero boundary conditions, but nonhomogeneous jump conditions, which are modified to accommodate the change due to the first step. The sum of the solutions in the two steps is the solution of Theorem A.2.

System (A.4) - A.9) bears some resemblance to the linear systems in $\S 3$. However, in regular layers, the term $u_{\xi \xi}=\epsilon^{2} u_{x x}$ can not be dropped to make an algebraicdifferential system. Because of this, even the relatively easier STEP ONE is not trivial to carry out. The point is we need to find a solution in each layer that is bounded uniformly by $\left(F^{i}, G^{i}\right)$ as the length of intervals approaches infinity in the $\xi$ scale when $\epsilon \rightarrow 0$. The procedure of performing STEP ONE is discussed in [18] and will be skipped in this paper.

To accomplish STEP TWO, based on Lemma A.1 we will simplify the system to make it easy to solve. Eventually, the system is reduced to the (BVPIC) which is known to have a solution.

In regular layers, by the change of variable $u=y-\left(f_{u}^{i}\right)^{-1} f_{v}^{i} v$, (A.5) becomes

$$
v_{x x}+g_{u}^{i} y+\left[g_{v}^{i}-g_{u}^{i}\left(f_{u}^{i}\right)^{-1} f_{v}^{i}\right] v=0 .
$$

The idea is if $y=0$, we are on the slow manifold of the linear system, so that the deviation $y$ must be small. If we drop $g_{u}^{i} y$, then the system to solve in regular layer is

$$
\begin{aligned}
& u_{\xi \xi}+f_{u}^{i} u+f_{v}^{i} v=0, \\
& v_{x x}+\left[g_{v}^{i}-g_{u}^{i}\left(f_{u}^{i}\right)^{-1} f_{v}^{i}\right] v=0 .
\end{aligned}
$$

In singular layers, we convert $v_{x x}=0$ into a system $v_{x}=w, w_{x}=0$, and approximate it by $v_{x}=0, w_{x}=0$. Then in singular layers

$$
\begin{gathered}
u_{\xi \xi}+f_{u}^{i} u+f_{v}^{i} v=0, \\
v_{x}=0, w_{x}=0 .
\end{gathered}
$$

Recall that by the iteration method, all we need is to solve the system approximately with small errors. After solving for $(u, v, w)$, we can show a posteriori that $g_{u}^{i} y$ is small in $L^{1}$ norm, see [18] for a proof, and $|w|_{L^{1}} \leq C \epsilon^{\beta}|w|_{L^{\infty}}$ is also small.

We look for solutions of a system consisting of (A.10)-A.13) plus the boundary conditions (A.8) and the jump conditions (A.9). The next step is to reduce the system to the (BVPIC) as in $\S 3$.

First we solve for $v$ in regular layers from (A.11). We need jump conditions for $v$ between two adjacent regular layers, one before the other after the $\ell$ th internal layer $I^{i}=I^{2 \ell+1}$. Observe that from (A.13),$\left(v^{i}, w^{i}\right)$ are constants in $I^{i}$. If we 
Recall that $\left[v_{0 x}^{R}\right]\left(x_{0}^{\ell}\right)=0$, from the jump conditions (A.9), we find that

$$
\begin{aligned}
& v^{i+1}\left(a^{i}\right)-v^{i-1}\left(a^{i-1}\right)=J_{3}^{i}+J_{3}^{i-1}, \\
& w^{i+1}\left(a^{i}\right)-w^{i-1}\left(a^{i-1}\right)=J_{4}^{i}+J_{4}^{i-1}+\Delta x^{\ell}\left[w_{0 x}^{R}\right]\left(x_{0}^{\ell}\right), \\
& A_{0} v_{x}^{2}\left(a^{1}\right)+B_{0} v^{2}\left(a^{1}\right)=A_{0} J_{4}^{1}+B_{0} J_{3}^{1}, \\
& A_{1} v_{x}^{2 r+2}\left(a^{2 r+2}\right)+B_{1} v^{2 r+2}\left(a^{2 r+2}\right)=-A_{1} J_{4}^{2 r+1}-B_{1} J_{3}^{2 r+1} .
\end{aligned}
$$

Let us turn to the $u$ equations in singular and regular layers. Consider A.10 and A.12 with Neumann boundary conditions. For the jump conditions on $\left(u, u_{x}\right)$, consider the $\ell$ th singular layer $I^{i}, i=2 \ell+1$.

$$
\begin{aligned}
& u^{i+1}\left(a^{i}\right)-u^{i}\left(a^{i}\right)=H_{1}^{i}:=J_{1}^{i}-u_{0 x}^{R, \ell+1}\left(x_{0}^{\ell}+\right) \Delta x^{\ell}, \\
& u_{x}^{i+1}\left(a^{i}\right)-u_{x}^{i}\left(a^{i}\right)=H_{2}^{i}:=J_{2}^{i}+u_{0 x}^{R, \ell}\left(x_{0}^{\ell}-\right) \Delta x^{\ell} .
\end{aligned}
$$

Equations for $u$ have the property that in the two boundary layers and all the regular layers, $u_{\xi \xi}+f_{u}^{i} u=0$ has exponential dichotomies in $I^{i}=\left(\xi^{i-1}, \xi^{i}\right)$. In each internal layer $I^{i}=I^{2 \ell+1}, \ell=1, \ldots, r, u_{\xi \xi}+f_{u}^{i} u=0$ has exponential dichotomies only on the two half-subintervals of $I^{i}$. By having an exponential dichotomy for a second order equation, we mean that the corresponding first order system on $\left(u, u_{\xi}\right)$ has an exponential dichotomy. The constants and exponents of the dichotomies do not depend on $\epsilon$ or the length of the intervals, which approaches infinity as $\epsilon \rightarrow 0$. Let the projections to stable and unstable spaces in $I^{i}$ be $P_{s}^{i}(\xi)$ and $P_{u}^{i}(\xi)$. The projections in internal layers have a jump at the middle of the interval $I^{i}$ since the dichotomies only exist on half of each $I^{i}$.

If $H_{j}^{i}, j=1,2$, is given, the system with jump conditions and exponential dichotomies described above has been studied in [29, 31, 30, 18. The problem to solve is similar to the classical shadowing lemma except for the lack of exponential dichotomies in the whole internal layers. Assuming at $\xi^{i}, \mathcal{R} P_{u}^{i}\left(\xi^{i}\right) \oplus \mathcal{R} P_{s}^{i+1}\left(\xi^{i}\right)$ which can be verified in our system, we have the unique splitting $\left(H_{1}^{i}, H_{2}^{i}\right)^{\tau}=\phi_{s}^{i+1}-\phi_{u}^{i}$ where $\phi_{s}^{i+1} \in \mathcal{R} P_{s}^{i+1}\left(\xi^{i}\right)$ and $\phi_{u}^{i} \in \mathcal{R} P_{u}^{i}\left(\xi^{i}\right)$. Denote $\phi_{u}^{i}:=Q_{u}^{i}\left(H_{1}^{i}, H_{2}^{i}\right)^{\tau}, \phi_{s}^{i+1}:=$ $Q_{s}^{i}\left(H_{1}^{i}, H_{2}^{i}\right)^{\tau}$. The system for $u$ can be approximated by a local boundary value problem in $I^{i}$ :

$$
\begin{array}{r}
u_{\xi \xi}+f_{u}^{i} u+f_{v}^{i} v=0, \\
P_{s}^{i}\left(\xi^{i-1}\right)\left(u\left(\xi^{i-1}\right), u_{x}\left(\xi^{i-1}\right)\right)=\phi_{s}^{i}, \\
P_{u}^{i}\left(\xi^{i}\right)\left(u\left(\xi^{i}\right), u_{x}\left(\xi^{i}\right)\right)=\phi_{u}^{i} .
\end{array}
$$

In regular layers, and in the two boundary layers, the above always has a solution for any continuous or $L^{1}$ function $v(\xi)$ and any vectors $\left(\phi_{s}^{i}, \phi_{u}^{i}\right)$. In internal layers, $v$ is constant, If $\Psi=\left(-\dot{\psi}^{\ell}, \psi^{\ell}\right)$, where $\psi^{\ell}$ is the solution to the adjoint equation as in $\S 2$. To have a solution $u$ in $I^{i}=I^{2 \ell+1}$, which is $\left(-\epsilon^{\beta-1}, \epsilon^{\beta-1}\right)$ using local coordinate, a Melnikov type condition must be satisfied, see Lemma 2.4.

$$
\begin{aligned}
& \int_{-\beta^{-1}}^{\beta^{-1}} \psi^{\ell}(\xi) f_{v} v^{i} d \xi=\Psi^{i}\left(\xi^{i}\right) \phi_{u}^{i}-\Psi^{i}\left(\xi^{i-1}\right) \phi_{s}^{i} \\
& \quad=\Psi^{i}\left(\xi^{i}\right) Q_{u}^{i}\left(H_{1}^{i}, H_{2}^{i}\right)^{\tau}-\Psi^{i}\left(\xi^{i-1}\right) Q_{s}^{i-1}\left(H_{1}^{i-1}, H_{2}^{i-1}\right)^{\tau} .
\end{aligned}
$$

It is now clear, based on $\Psi^{i}(\xi)$ being exponentially small as $\xi \rightarrow \infty$, we can drop the $\Delta x^{\ell}$ in the $\left(H_{1}^{i}, H_{2}^{i}\right)$ terms, defined in (A.18). The right hand side is approximated by given terms involving only $\left(J_{1}^{i-1}, J_{2}^{i-1}, J_{1}^{i}, J_{2}^{i}\right)$. If we denote $\mathbf{n}_{0}^{\ell}:=$ 
$\int_{-\beta^{-1}}^{\beta^{-1}} f_{v}^{i} \psi^{\ell}(\xi) d \xi$ and use the jump condition $v^{i+1}\left(a^{i}\right)-v^{i}\left(a^{i}\right)=J_{3}^{i}-w_{0}^{R}\left(x_{0}^{\ell}\right) \Delta x^{\ell}$, we have a condition on $v^{i+1}\left(a^{i}\right)$ :

$$
\mathbf{n}_{0}^{\ell} \cdot\left(v^{i+1}\left(a^{i}\right)+w_{0}^{R}\left(x_{0}^{\ell}\right) \Delta x^{\ell}\right)=\Psi^{i}\left(\xi^{i}\right) Q_{u}^{i}\left(J_{1}^{i}, J_{2}^{i}\right)^{\tau}-\Psi^{i}\left(\xi^{i-1}\right) Q_{s}^{i-1}\left(J_{1}^{i-1}, J_{2}^{i-1}\right)^{\tau} .
$$

In the simplified system, the $v$ variable in regular layers must satisfy (A.19), with jump conditions (A.14), (A.15) and boundary conditions (A.16), A.17).

If we shrink the singular layer to a point $x_{0}^{\ell}, 0 \leq \ell \leq r+1$, and move $a^{2 \ell}$ and $a^{2 \ell+1}$ to $x_{0}^{\ell}$, and approximate the $\mathbf{n}_{0}^{\ell}$ by $\mathbf{n}^{\ell}=\int_{-\infty}^{\infty} f_{v}^{\bar{i}} \psi^{\ell}(\xi) d \xi$, then (A.19) is approximated by

$$
\mathbf{n}^{\ell} \cdot\left(v^{i+1}\left(x_{0}^{\ell}+\right)+w_{0 x}^{R}\left(x_{0}^{\ell}+\right) \Delta x^{\ell}\right)=\text { given terms. }
$$

This is precisely the third equation in (BVPIC). The boundary conditions become the second equation in (BVPIC) and the jump conditions the last two equations in (BVPIC). According to Lemma 3.2 the modified system has a unique solution. If we solve this (BVPIC) and map the solution in each $\left(x_{0}^{\ell-1}, x_{0}^{\ell}\right)$ by a near identity map to $\left(a^{2 \ell-1}, a^{2 \ell}\right)$, we have a good approximation of the $v$ in regular layers. The error of the approximation approaches zero as $\epsilon \rightarrow 0$. By Lemma A.1, this means that the system for the $v$ variable in regular layers has a unique solution.

The $v$ in singular layers can be obtained by jump conditions to their neighboring regular layers. Finally, since the Melnikov type condition is satisfied in each internal layer, $u$ with boundary and jump condition can be obtained.

Once the linear system has been solved, the nonlinear variational system can be solved by a contraction mapping principle. We summarize the result below:

Theorem A.3. For any integer $m \geq 0$, there exists $\epsilon_{0}>0$ such that if $0<\epsilon<\epsilon_{0}$, there exists a unique internal layer solution $\left(u_{\text {exact }}, v_{\text {exact }}\right)$ near the formal approximation $\left(u_{a p}, v_{a p}\right)$. The internal layer solution has exact layer positions (determined by some phase condition) $x_{\text {exact }}^{\ell}, 1 \leq \ell \leq r$, that is near $x_{a p}^{\ell}$. Moreover,

$$
\left|u_{\text {exact }}-u_{a p}\right|+\left|v_{\text {exact }}-v_{a p}\right|+\sum_{\ell}\left|x_{\text {exact }}^{\ell}-x_{a p}^{\ell}\right| \leq C \epsilon^{\beta(m+1)}, \quad 0<\beta<1 .
$$

Formal approximation of critical eigenvalue and eigenfunctions. By truncating the formal series of eigenvalues and eigenfunctions as above, we can show that $\lambda_{a p}(\epsilon)$ and $\left(U_{a p}, V_{a p}\right)$ are approximations of eigenvalue and eigenfunctions with small residual in each $I^{i}$ and jump errors between layers.

If we let $\left(-F^{i},-G^{i}\right)$ be the residual error of the approximation of eigenvalue and eigenfunctions in $I^{i}$, then

$$
\begin{aligned}
& -\lambda_{a p} U_{a p}+U_{a p, \xi \xi}+f_{u}^{i} \text { (exact) } U+f_{v}^{i} \text { (exact) } V=-F^{i}, \\
& -\lambda_{a p} V_{a p}+V_{a p, x x}+g_{u}^{i} \text { (exact) } U+g_{v}^{i} \text { (exact) } V=-G^{i}, \quad 1 \leq i \leq 2 r+3 .
\end{aligned}
$$

Here $f_{u}^{i}($ exact $)=f_{u}\left(u_{\text {exact }}, v_{\text {exact }}\right)$ in regular layers, etc. One can verify that $\left|F^{i}\right|$ and $\left|G^{i}\right|$ satisfy estimates (A.2) with perhaps different constants $C$.

When $\Delta^{\ell}=0, \ell=1, \ldots, r$, the jump errors between layers are defined as

$$
\begin{array}{ll}
U_{a p}^{i+1}\left(a_{0}^{i}\right)-U_{a p}^{i}\left(a_{0}^{i}\right)=-J_{1}^{i}, & U_{a p, x}^{i+1}\left(a_{0}^{i}\right)-U_{a p, x}^{i}\left(a_{0}^{i}\right)=-J_{2}^{i}, \\
V_{a p}^{i+1}\left(a_{0}^{i}\right)-V_{a p}^{i}\left(a_{0}^{i}\right)=-J_{3}^{i}, & V_{a p, x}^{i+1}\left(a_{0}^{i}\right)-V_{a p, x}^{i}\left(a_{0}^{i}\right)=-J_{4}^{i} .
\end{array}
$$

They satisfy A.3 with perhaps different constants $C$. 
Existence of critical eigenvalue-eigenfunctions. We outline the proof of the following result:

Theorem A.4. For any integer $m \geq 0$ and $1 \leq k \leq r$, there exists $\epsilon_{0}>0$ such that if $0<\epsilon<\epsilon_{0}$, then there exists a unique eigenvalue-eigenfunction triplet $\left(\lambda_{\text {exact }}, U_{\text {exact }}, V_{\text {exact }}\right)$ near $\left(\lambda_{a p}^{k}(\epsilon), U_{a p}, V_{a p}\right)$. Moreover,

$$
\left|\lambda_{a p}^{k}-\lambda_{\text {exact }}\right|+\left|U_{\text {ap }}-U_{\text {exact }}\right|+\left|V_{a p}-V_{\text {exact }}\right|=O\left(\epsilon^{(m+1) \beta}\right) .
$$

When we construct $U_{a p}$, an undetermined term $\epsilon^{m} c_{m}^{\ell} \dot{q}^{\ell}$ can be added in the $\ell$ th singular layer. The vector $\left\{c_{m}^{\ell}\right\}_{\ell=1}^{r}$ will be determined now. Let an exact solution be

$$
\begin{aligned}
\lambda_{\text {exact }} & =\lambda_{a p}^{k}+\epsilon^{m+1} \lambda, & & \\
U_{\text {exact }} & =U_{a p}+\epsilon^{m+1} U, & & \text { in regular layers, } \\
U_{\text {exact }} & =U_{a p}+\epsilon^{m} c_{m}^{\ell} \dot{q}^{\ell}+\epsilon^{m+1} U, & & \text { in the } \ell \text { th singular layer, } \\
V_{\text {exact }} & =V_{a p}+\epsilon^{m+1} V, & & \text { in regular and singular layers. }
\end{aligned}
$$

In regular layers,

$$
\begin{aligned}
U_{\xi \xi}+f_{u}^{i} U+f_{v}^{i} V & =F^{i}(\xi)+M^{i}(U, V, \lambda, \epsilon), \\
V_{x x}+g_{u}^{i} U+g_{v}^{i} V & =G^{i}(x)+N^{i}(U, V, \lambda, \epsilon) .
\end{aligned}
$$

In the $\ell$ th singular layer,

$$
\begin{aligned}
& -\lambda_{1} c_{m}^{\ell} \dot{q}^{\ell}-\lambda c_{0}^{\ell} \dot{q}^{\ell}+U_{\xi \xi}+f_{u}^{i} U+f_{v}^{i} V+f_{u u}^{i} c_{m}^{\ell} \dot{q}^{\ell} u_{1}+f_{u v}^{i} c_{m}^{\ell} \dot{q}^{\ell} v_{1} \\
& \quad=F^{i}(\xi)+M^{i}\left(U, V, \lambda, c_{m}^{\ell}, \epsilon\right), \\
& V_{\xi}=\epsilon W, \quad W_{\xi}=-g_{u}^{i} c_{m}^{\ell} \dot{q}^{\ell}+G^{i}(x)+N^{i}\left(U, V, \lambda, c_{m}^{\ell}, \epsilon\right) .
\end{aligned}
$$

The nonlinear terms satisfy

$$
\left|M^{i}\right|+\left|N^{i}\right|_{L^{1}} \leq C\left(|U|^{2}+|V|^{2}+|\lambda|^{2}+\left|c_{m}^{\ell}\right|^{2}+\epsilon\left(|U|+|V|+|\lambda|+\left|c_{m}^{\ell}\right|\right)\right) .
$$

In regular layers, the nonlinear terms satisfy a similar estimate.

There are also boundary conditions at $x=0,1$ and jump conditions at $\left\{a^{i}\right\}$ to be satisfied. The nonlinear system can be solved by a contraction method if the following linear system can be solved.

In regular layers,

$$
\begin{aligned}
& U_{\xi \xi}+f_{u}^{i} U+f_{v}^{i} V=F^{i}(\xi), \\
& V_{x x}+g_{u}^{i} U+g_{v}^{i} V=G^{i}(x) .
\end{aligned}
$$

In the $\ell$ th singular layer,

$$
\begin{aligned}
& -\lambda_{1} c_{m}^{\ell} \dot{q}^{\ell}-\lambda c_{0}^{\ell} \dot{q}^{\ell}+U_{\xi \xi}+f_{u}^{i} U+f_{v}^{i} V+f_{u u}^{i} c_{m}^{\ell} \dot{q}^{\ell} u_{1}+f_{u v}^{i} c_{m}^{\ell} \dot{q}^{\ell} v_{1}=F^{i}(\xi), \\
& V_{\xi}=0, \quad W_{\xi}=-g_{u}^{i} c_{m}^{\ell} \dot{q}^{\ell}+G^{i}(x) .
\end{aligned}
$$

The boundary and jump conditions are

$$
\begin{aligned}
U_{x}^{1}\left(a^{0}\right)=U_{x}^{2 \ell+3}\left(a^{2 \ell+3}\right) & =0, & & A_{j} V_{x}(j)+B_{j} V(j)=0, \\
U^{i+1}\left(a^{i}\right)-U^{i}\left(a^{i}\right) & =J_{1}^{i}, & & U_{x}^{i+1}\left(a^{i}\right)-U_{x}^{i}\left(a^{i}\right)=J_{2}^{i}, \\
V^{i+1}\left(a^{i}\right)-V^{i}\left(a^{i}\right) & =J_{3}^{i}, & & V_{x}^{i+1}\left(a^{i}\right)-V_{x}^{i}\left(a^{i}\right)=J_{4}^{i} .
\end{aligned}
$$

The linear system is again solved in two steps. First we solve the nonhomogeneous system in each layer with no concern of jump conditions. This is done in 
[18 and will be skipped here. In the rest of this section, we solve the system with $F^{i}=0, G^{i}=0$. The iteration method as Lemma A.1 will be used.

Integrating in the $\ell$ th singular layer, we have

$$
\begin{aligned}
V^{i}\left(a^{i}\right)-V^{i}\left(a^{i-1}\right) & =0, \\
W^{i}\left(a^{i}\right)-W^{i}\left(a^{i-1}\right) & =c_{m}^{\ell}\left(g\left(q^{\ell}\left(-\epsilon^{\beta-1}\right), v\right)-g\left(q^{\ell}\left(\epsilon^{\beta-1}\right), v\right)\right) \approx c_{m}^{\ell} \mathcal{M}^{\ell} .
\end{aligned}
$$

Here $\mathcal{M}^{\ell}:=g\left(q^{\ell}(-\infty), v\right)-g\left(q^{\ell}(\infty), v\right)$ as defined in $\S 4$. It is now clear that the jump of $(V, W)$ between the two regular layers next to $I^{2 \ell+1}$ are approximately

$$
\begin{aligned}
V^{i+1}\left(a^{i}\right)-V^{i-1}\left(a^{i-1}\right) & =J_{3}^{i}+J_{3}^{i-1}, \\
W^{i+1}\left(a^{i}\right)-W^{i-1}\left(a^{i-1}\right) & =J_{4}^{i}+J_{4}^{i-1}+c_{m}^{\ell} \mathcal{M}^{\ell} .
\end{aligned}
$$

Using the change of variable $U=Y-\left(f_{u}^{i}\right)^{-1} f_{v}^{i} V$, in regular layers

$$
V_{x x}-\left[g_{u}^{i}\left(f_{u}^{i}\right)^{-1} f_{v}^{i}-g_{v}^{i}\right] V+g_{u}^{i} Y=0 .
$$

Dropping the small term $g_{u}^{i} Y$, and also observing that $a^{i}$ and $a^{i-1}$ are $\epsilon^{\beta}$ close to $x_{0}^{\ell}$, an approximate system of $V$ has the form

$$
\begin{aligned}
& V_{x x}-\left[g_{u}^{i}\left(f_{u}^{i}\right)^{-1} f_{v}^{i}-g_{v}^{i}\right] V=0, \\
& {[V]\left(x_{0}^{\ell}\right)=J_{3}^{i}+J_{3}^{i-1},} \\
& {\left[V_{x}\right]\left(x_{0}^{\ell}\right)=J_{4}^{i}+J_{4}^{i-1}+c_{m}^{\ell} \mathcal{M}^{\ell},}
\end{aligned}
$$

with homogeneous boundary conditions. The solution can be written as $V=$ $c_{m}^{\ell} V_{c}^{\ell}+$ given terms, $V_{c}^{\ell}$ as in $\S 4$.

To determine $c_{m}^{\ell}$, plug $V$ into the $U$ equation in internal layers. In order to have a solution in $I^{2 \ell+1}$, we have a Melnikov type condition

$$
\begin{aligned}
& \int_{-\beta^{-1}}^{\beta^{-1}}\left\langle\psi^{\ell}(\xi),-\lambda_{1} c_{m}^{\ell} \dot{q}^{\ell}-\lambda c_{0}^{\ell} \dot{q}^{\ell}+f_{v}^{i} V+f_{u u}^{i} c_{m}^{\ell} \dot{q}^{\ell} u_{1}+f_{u v}^{i} c_{m}^{\ell} \dot{q}^{\ell} v_{1}\right\rangle d \xi \\
& \quad=\text { given terms. }
\end{aligned}
$$

Replacing the domain of integration by $(-\infty, \infty)$, using (4.19), and recalling that $\mathbf{n}^{\ell}=\int_{-\infty}^{\infty} f_{v} \psi^{\ell} d \xi$, we finally have

$$
\lambda_{1} c_{m}^{\ell}+\lambda c_{0}^{\ell}=\mathbf{n}^{\ell}\left(\sum_{1}^{r} c_{m}^{i} V_{c}^{i}-c_{m}^{\ell} w_{0}^{R}\left(x_{0}^{\ell}\right)\right)+\text { given terms. }
$$

With $A$ being the coupling matrix, the above can be written as

$$
\left(A-\lambda_{1} I\right) \mathbf{c}_{m}=\lambda \mathbf{c}_{0}+\text { given terms. }
$$

Here we denote a $r$-vector $\left(c^{1}, \ldots, c^{r}\right)^{\tau}$ by $\mathbf{c}$. Since $\lambda_{1}$ is a simple eigenvalue and $\mathbf{c}_{0}$ is not in the range of $A-\lambda_{1} I$, there exists a unique $\lambda$ such that the above can be solved for a unique vector $\mathbf{c}_{m}$. After that, we can determine a unique $U^{2 \ell+1} \perp \dot{q}^{\ell}$ in each singular layer. Approximations for $(U, V)$ in regular layers can also be solved accordingly. The exact solution of $\left(U, V, \lambda,\left\{c_{m}^{\ell}\right\}\right)$ is obtained by the iteration method as in Lemma A.1.

\section{ACKNOWLEDGMENT}

The author is grateful for the referee's suggestion that helped to improve the presentation of the paper. 


\section{REFERENCES}

[1] S. B. Angenent, J. Mallet-Paret, and L. A. Peletier, Stable transition layers in a semilinear boundary value problem, J. Differential Equations, 67 (1987), 212-242. MR 88d:34018

[2] P. Bates, P. Fife, R. Gardner and C.K.R.T. Jones, the existence of traveling wave solutions of a generalized phase field model, SIAM J. Math. Anal. 28 (1997), 60-93. MR 97m:35284

[3] F. Battelli, Heteroclinic orbits in singular systems: a unifying approach, J. Dyn. Diff. Eqns. 6 (1994), 147-173. MR 95d:34068

[4] Amitabha Bose, Symmetric and antisymmetric pulses in parallel coupled nerve fibers, SIAM J. Appl. Math. 55 (1995), 1650-1674. MR 96i:35060

[5] J. Carr and R. L. Pego, Metastable patterns in solutions of $u_{t}=\epsilon^{2} u_{x x}-f(u)$, Comm. Pure. Appl. Math. 42 (1989), 523-576. MR 90f:35091

[6] S.-N. Chow, J. K. Hale and J. Mallet-Paret, An example of bifurcation to homoclinic orbits, J. Differential Equations 37 (1980), 351-373. MR 81m:58056

[7] W. A. Coppel, Dichotomies in stability theory, in "Lecture Notes in Mathematics, Vol. 629", Springer-Verlag, New York/Berlin, 1978. MR 58:1332

[8] A. Doelman and V. Rottschäfer, Singularly perturbed and nonlocal modulation equations for systems with interacting instability mechanisms, J. Nonlinear Sci. 7 (1997), 371-409. MR 98m:35190

[9] N. Fenichel, Persistence and smoothness of invariant manifolds for flows, Indiana Univ. Math. Journal, 21:193-226, 1971. MR 44:4313

[10] N. Fenichel, Geometric singular perturbation theory for differential equations, J. Differential Equations 31 (1979), 53-98. MR 80m:58032

[11] P. C. Fife, Transition layer in singularly perturbed problems, J. Differential Equations, 15 (1974), 77-105. MR 48:9002

[12] P. C. Fife, Boundary and interior transition layer phenomena for pairs of second-order differential equations, J. Math. Anal. App. 54 (1976), 497-521. MR 54:7978

[13] P. C. Fife and L. Hsiao, The generating of solutions of internal layers, J. Nonlinear Anal. TMA 12 (1988), 19-41. MR 89c:35078

[14] H. Fujii \& Y. Hosono, Neumann layer phenomena in nonlinear diffusion systems, Lecture Notes in Num. Anal. 6 (1983), 21-38. MR 87a:35008

[15] G. Fusco and J. Hale, Slow motion manifolds, dormant instability, and singular perturbations, J. Dynamics and Differential Equations 1 (1989), 75-94. MR 90i:35131

[16] J. Guckenheimer, Dynamical Systems, Birkhäuser, Boston, 1980. MR 82g:58065

[17] M. Hayes, T. Kaper, N. Kopell, K. Ono, On the application of geometric singular perturbation theory to some classical two point boundary value problems, Internat. J. Bifur. Chaos Appl. Sci. Engrg. 8 (1998), 189-209. MR 99c:34119

[18] J.K. Hale \& X.-B. Lin, Multiple internal layer solutions generated by spatially oscillatory perturbations, J. Differential Eqn., 154 (1999), 364-418. MR 2000c:35097

[19] J. Hale \& K. Sakamoto, Existence and stability of transition layers, Japan J. Appl. Math. 5 (1988), 367-405. MR 90a:35112

[20] E.J. Hinch, Perturbation methods, Cambridge University Press, 1991. MR 93a:34002

[21] F. C. Hoppensteadt, Singular perturbations on the infinite interval, Trans. Amer. Math. Soc., 123:521-535, 1966. MR 33:2900

[22] F. C. Hoppensteadt, Properties of solutions of ordinary differential equations with small parameters, Comm. Pure Appl. Math., 24:807-840, 1971. MR 44:5576

[23] Y. Hosono \& M. Mimura, Singular perturbation approach to traveling waves in competing and diffusing species models, J. Math. Kyoto Univ. 22-23 (1982) 435-461. MR 84d:35014

[24] C. Jones, Geometric singular perturbation theory, Lectures Notes in Math. Springer-Verlag, Berlin, 1069:44-118, 1995. MR 97e:34105

[25] C. K. R. T. Jones and N. Kopell, Tracking invariant manifolds with differential forms in singularly perturbed systems, J. Differential Equations, 108 (1994), 64-89. MR 95c:34085

[26] C. Jones and J. Rubin, Existence of standing pulse solutions of an inhomogeneous reactiondiffusion system, J. Dyn. Diff. Eqns. 10 (1998), 1-35. MR 99b:35094

[27] C. K. R. T. Jones, T. Kaper and N. Kopell, Tracking invariant manifolds up to exponentially small errors, SIAM J. Math. Anal. 27, 558-577. MR 97a:58167

[28] H. Kokubu, Y. Nishiura and H. Oka, Heteroclinic and homoclinic bifurcation in bistable reaction diffusion systems, J. Diff. Eqn. 86 (1990), 260-341. MR 92a:58128 
[29] X-B. Lin, Shadowing lemmas and singularly perturbed boundary value problems, SIAM J Appl. Math., 49 (1989), 26-54. MR 90a:34126

[30] X-B. Lin, Heteroclinic bifurcation and singularly perturbed boundary value problems, J. Differential Equations, 84 (1990), 319-382. MR 91d:34055

[31] X.-B. Lin, Using Melnikov's method to solve Silnikov's problems, Proc. Royal Soc. Edinburgh Sect. A 116 (1990), 295-325. MR 92b:58195

[32] X-B. Lin, Local and global existence of multiple wave near formal approximations, Progress in Nonlinear Diff Eqns \& Their Appl. 19 (1996), 385-404, Birkhäuser-Verlag. MR 97b:35019

[33] X-B. Lin, Asymptotic expansion for layer solutions of a singularly perturbed reactiondiffusion system, Trans. AMS. 348 (1996), 713-753. MR 96g:35023

[34] X-B. Lin, Shadowing matching errors for wave front like solutions, J. Differential Equations, 129 (1996), 403-457. MR 97f:35022

[35] H. Meinhardt, Models of biological pattern formation. Academic Press, 1982.

[36] M. Mimura, M. Tabata \& Y. Hosono, Multiple solutions of two-point boundary value problems of Neumann type with a small parameter, SIAM J. Math. Anal., 11 (1980), 613-631. MR 81g:34025

[37] J. D. Murray, Mathematical Biology. Springer-Verlag, 1989. MR 90g:92001

[38] Y. Nishiura, Coexistence of infinitely many stable solutions to reaction diffusion systems in the singular limit, Dynamics Reported, New Series, 3, (1994), 25-103,

[39] Y. Nishiura \& H. Fujii, Stability of singularly perturbed solutions to systems of reactiondiffusion equations, SIAM J. Math. Anal. 18 (1987), 1726-1770. MR 88j:35089

[40] Y. Nishiura \& H. Fujii, SLEP method to the stability of singularly perturbed solutions with multiple transition layers in reaction-diffusion systems, in "Dynamics of Infinite Dimensional Systems," Eds. J. Hale \& S.-N. Chow, Springer-Verlag, 1987. MR 89b:35009

[41] R. E. O'Malley, Singular perturbation methods for ordinary differential equations, Applied mathematical sciences, 89, 1990. MR 92i:34071

[42] K. J. Palmer, Exponential dichotomies and transversal homoclinic points, J. Differential Equations, 55 (1984), 225-256. MR 89j:58060

[43] K. J. Palmer, Transversal heteroclinic points and Cherry's example of a nonintegrable hamiltonian system. J. Diff. Eqs., 65 (1986), 321-360. MR 88a:58078

[44] R. J. Sacker, The splitting index for linear differential systems, J. Differential Equations 33 (1979), 368-405. MR 81f:34020

[45] R. J. Sacker and G. R. Sell, Existence of dichotomies and invariant splittings for linear differential equations, II, III. J. Differential Equations, 22 (1976), 478-496, 497-522. MR 55:13494 MR 55:13495

[46] K. Sakamoto, Construction and stability analysis of transition layer solutions in reactiondiffusion systems, Tohoko Math J. 42 (1990), 17-44. MR 91k:35127

[47] P. Szmolyan, Transversal heteroclinic and homoclinic orbits in singular perturbation problems, J. Differential Equations, 92:252-281, 1991. MR 92e:58185

[48] S. K. Tin, N. Kopell \& C. K. R. T. Jones, Invariant manifolds and singularly perturbed boundary value problems, SIAM J. Numer. Anal. 31, (1994), 1558-1576. MR 96j:34098

[49] S. Wiggins, Global bifurcation and chaos, Springer-Verlag, New York, 1988. MR 89m:58057

Department of Mathematics, North Carolina State University, Raleigh, North CarOLINA 27695-8205

E-mail address: xblin@xblsun.math.ncsu.edu 